\title{
Birational maps preserving the contact structure on $\mathbb{P}_{\mathbb{C}}^{3}$
}

\author{
By Dominique Cerveau and Julie Déserti
}

(Received Aug. 6, 2016)

\begin{abstract}
We study the group of polynomial automorphisms of $\mathbb{C}^{3}$ (resp. birational self-maps of $\mathbb{P}_{\mathbb{C}}^{3}$ ) that preserve the contact structure.
\end{abstract}

\section{Introduction.}

In this article we work on the group of birational maps that preserve contact structures on $\mathbb{P}_{\mathbb{C}}^{3}$. On $\mathbb{P}_{\mathbb{C}}^{3}$ there is, up to automorphisms, only one (non-singular) contact structure given in homogeneous coordinates by the 1 -form $\widetilde{\vartheta}=z_{0} \mathrm{~d} z_{1}-z_{1} \mathrm{~d} z_{0}+z_{2} \mathrm{~d} z_{3}-z_{3} \mathrm{~d} z_{2}$. In $\mathbb{C}^{3}$ there is the Darboux 1-form $\omega=z_{0} \mathrm{~d} z_{1}+\mathrm{d} z_{2}$ that is the standard local model of contact forms; it thus defines a holomorphic contact structure on $\mathbb{C}^{3}$ that extends to $\mathbb{P}_{\mathbb{C}}^{3}$ meromorphically. Note that $\omega$ has poles of order 3 along the hyperplane $z_{3}=0$. We denote by $\mathrm{c}(\omega)$ the (meromorphic) contact structure induced on $\mathbb{P}_{\mathbb{C}}^{3}$ by $\omega$. Let us remark that actually $\omega$ is birationally conjugate to $\widetilde{\vartheta}_{\mid z_{3}=1}$ (more precisely they are conjugate via a polynomial automorphism in the affine chart $z_{3}=1$ ). As a result the group of birational maps that preserve these structures are conjugate; since it is more convenient to work with $\omega$ than with $\widetilde{\vartheta}$ we will focus on $\omega$.

The contact geometry has a long story. The Darboux local model $z_{0} \mathrm{~d} z_{1}+\mathrm{d} z_{2}$ is related to the formalization of $z_{0}=-\mathrm{d} z_{2} / \mathrm{d} z_{1}$. For instance if $\mathcal{S}$ is a surface in $\mathbb{C}^{3}$ given by $F\left(z_{0}, z_{1}, z_{2}\right)=0$ then the restriction of $\omega$ to $\mathcal{S}$ corresponds to the implicit differential equation $F\left(-\partial z_{2} / \partial z_{1}, z_{1}, z_{2}\right)=0$. A birational self-map of $\mathbb{P}_{\mathbb{C}}^{3}$ which preserves the contact structure (i.e., which sends the 1 -form $z_{0} \mathrm{~d} z_{1}+\mathrm{d} z_{2}$ viewed in the affine chart $z_{3}=1$ onto a multiple of $z_{0} \mathrm{~d} z_{1}+\mathrm{d} z_{2}$ by a rational function) is said to be a contact map. The space $\mathbb{C}^{3}$ with the contact form $\omega$ can be seen as an affine chart of the projectivization of the cotangent bundle $\mathrm{T}^{*} \mathbb{C}^{2}$ (equipped with the standard Liouville contact form). As a consequence there is a natural extension of any birational self-map of the $\left(z_{1}, z_{2}\right)$ plane $([\mathbf{2 3}])$

$\mathcal{K}: \operatorname{Bir}\left(\mathbb{P}_{\mathbb{C}}^{2}\right) \hookrightarrow \operatorname{Bir}\left(\mathbb{C}^{3}\right)_{\mathrm{c}(\omega)}, \quad\left(\phi_{1}, \phi_{2}\right) \mapsto\left(\frac{-\partial \phi_{2} / \partial z_{1}+\partial \phi_{2} / \partial z_{2} z_{0}}{\partial \phi_{1} / \partial z_{1}-\partial \phi_{1} / \partial z_{2} z_{0}}, \phi_{1}\left(z_{1}, z_{2}\right), \phi_{2}\left(z_{1}, z_{2}\right)\right)$

where $\operatorname{Bir}\left(\mathbb{C}^{3}\right)_{\mathrm{c}(\omega)}$ denotes the group of contact birational self-maps of $\mathbb{P}_{\mathbb{C}}^{3}$. The image of $\mathcal{K}$ is the Klein group $\mathscr{K}$. Klein conjectured that the group of contact maps is generated by $\mathscr{K}$ and the Legendre involution

2010 Mathematics Subject Classification. Primary 14E05; Secondary 14E07.

Key Words and Phrases. birational maps, polynomial automorphisms, Cremona group, contact structure. 


$$
\left(z_{0}, z_{1}, z_{2}\right) \mapsto\left(z_{1}, z_{0},-z_{2}-z_{0} z_{1}\right) .
$$

In 2008 Gizatullin proved this "conjecture" in the case in which the contact transformations are polynomial automorphisms of the affine space $([\mathbf{2 1}])$. The conjecture about generators of the contact group is still open in the birational case.

Let $\mathrm{G}$ be a subgroup of the group $\operatorname{Bir}\left(\mathbb{P}_{\mathbb{C}}^{n}\right)$ of birational self-maps of $\mathbb{P}_{\mathbb{C}}^{n}$, and let $\beta$ be a meromorphic $p$-form on $\mathbb{P}_{\mathbb{C}}^{n}$; denote by

$$
\mathrm{G}_{\beta}=\left\{\phi \in \mathrm{G} \mid \phi^{*} \beta=\beta\right\}
$$

the subgroup of elements of $\mathrm{G}$ that preserve the form $\beta$. In the same spirit for 1 -forms $\beta$ we set

$$
\mathrm{G}_{\mathrm{c}(\beta)}=\left\{\phi \in \mathrm{G} \mid \phi^{*} \beta \wedge \beta=0\right\} .
$$

We have the obvious inclusions $\mathrm{G}_{\beta} \subset \mathrm{G}_{\mathrm{c}(\beta)} \subset \mathrm{G}$.

We first describe the group $\operatorname{Aut}\left(\mathbb{C}^{3}\right)_{\mathrm{c}(\omega)}$ of polynomial automorphisms of $\mathbb{C}^{3}$ that preserve the contact structure:

Theorem 1.0.1. If $\eta$ is the form $\mathrm{d} \omega=\mathrm{d} z_{0} \wedge \mathrm{d} z_{1}$, then

$$
\operatorname{Aut}\left(\mathbb{C}^{3}\right)_{\omega} \simeq \operatorname{Aut}\left(\mathbb{C}^{2}\right)_{\eta} \ltimes \mathbb{C}, \quad \operatorname{Aut}\left(\mathbb{C}^{3}\right)_{\mathrm{c}(\omega)} \simeq \operatorname{Aut}\left(\mathbb{C}^{3}\right)_{\omega} \ltimes \mathbb{C}^{*}
$$

Hence, as Banyaga did in the context of contact diffeomorphisms of smooth real manifolds $([\mathbf{2}][\mathbf{3}][\mathbf{4}])$, one gets that the commutator of $\operatorname{Aut}\left(\mathbb{C}^{3}\right)_{\omega}\left(\operatorname{resp}\right.$. Aut $\left.\left(\mathbb{C}^{3}\right)_{\mathrm{c}(\omega)}\right)$ is perfect. Any automorphism of $\operatorname{Aut}\left(\mathbb{C}^{2}\right)$ is the composition of an inner automorphism and an automorphism of the field $\mathbb{C}$ (see $[\mathbf{1 6}])$. Following this idea we describe the group $\operatorname{Aut}\left(\operatorname{Aut}\left(\mathbb{C}^{3}\right)_{\omega}\right)$.

Danilov and Gizatullin proved that any finite subgroup of $\operatorname{Aut}\left(\mathbb{C}^{2}\right)$ is linearizable $([\mathbf{2 2}])$. We obtain a similar statement:

TheOrem 1.0.2. Any finite subgroup of $\operatorname{Aut}\left(\mathbb{C}^{3}\right)_{\mathrm{c}(\omega)}$ is linearizable via an element of $\operatorname{Aut}\left(\mathbb{C}^{3}\right)_{\mathrm{c}(\omega)}$.

We also deal with $\operatorname{Bir}\left(\mathbb{C}^{3}\right)_{\mathrm{c}(\omega)}$. If $\phi$ belongs to $\operatorname{Bir}\left(\mathbb{C}^{3}\right)_{\mathrm{c}(\omega)}$, then $\phi^{*} \omega=V(\phi) \omega$ where $V(\phi)$ is some rational function. In particular one gets a map $V$ from $\operatorname{Bir}\left(\mathbb{C}^{3}\right)_{\mathrm{c}(\omega)}$ to the set of rational functions in $z_{0}, z_{1}, z_{2}$ satisfying cocycle conditions: $V(\phi \circ \psi)=$ $(V(\phi) \circ \psi) \cdot V(\psi)$.

The equality $\phi^{*} \omega=V(\phi) \omega$ can be rewritten as the following system of PDE

$$
(\mathcal{S}) \begin{cases}\phi_{0} \partial \phi_{1} / \partial z_{0}+\partial \phi_{2} / \partial z_{0}=0, & \left(\star_{1}\right) \\ \phi_{0} \partial \phi_{1} / \partial z_{1}+\partial \phi_{2} / \partial z_{1}=V(\phi) z_{0}, & \left(\star_{2}\right) \\ \phi_{0} \partial \phi_{1} / \partial z_{2}+\partial \phi_{2} / \partial z_{2}=V(\phi) . & \left(\star_{3}\right)\end{cases}
$$

The first equation $\left(\star_{1}\right)$ has a special family of solutions: maps for which both $\phi_{1}$ and $\phi_{2}$ do not depend on $z_{0}$; we can then compute $\phi_{0}$ from the two other equations. Taking $\left(\phi_{1}, \phi_{2}\right)$ in $\operatorname{Bir}\left(\mathbb{P}_{\mathbb{C}}^{2}\right)$ we get in this way the group $\mathscr{K}$. 
Assume now that $\phi_{1}$ or $\phi_{2}$ depends on $z_{0}$ then both depend on it and $(\mathcal{S})$ implies the following equality

$$
\frac{\partial \phi_{2} / \partial z_{1}-z_{0} \partial \phi_{2} / \partial z_{2}}{\partial \phi_{2} / \partial z_{0}}=\frac{\partial \phi_{1} / \partial z_{1}-z_{0} \partial \phi_{1} / \partial z_{2}}{\partial \phi_{1} / \partial z_{0}} .
$$

Let us defined $\alpha$ the map from $\operatorname{Bir}\left(\mathbb{C}^{3}\right)_{\mathrm{c}(\omega)}$ into the set of rational functions in $z_{0}, z_{1}$ and $z_{2}$ by: $\alpha(\phi)=\infty$ if $\phi$ belongs to $\mathscr{K}$ and

$$
\alpha(\phi)=\frac{\partial \phi_{2} / \partial z_{1}-z_{0} \partial \phi_{2} / \partial z_{2}}{\partial \phi_{2} / \partial z_{0}}=\frac{\partial \phi_{1} / \partial z_{1}-z_{0} \partial \phi_{1} / \partial z_{2}}{\partial \phi_{1} / \partial z_{0}}
$$

otherwise.

If $\phi_{1}$ and $\phi_{2}$ are some first integrals of the rational vector field

$$
Z_{\phi}=\alpha(\phi) \frac{\partial}{\partial z_{0}}-\frac{\partial}{\partial z_{1}}+z_{0} \frac{\partial}{\partial z_{2}}
$$

one gets $\phi_{0}$ thanks to the first equation of $(\mathcal{S})$. Such $\phi$ is not necessary birational but only rational; nevertheless one gets a lot of contact birational self-maps in this way. Remark that since $\mathscr{K}$ (resp. $\left.\operatorname{Bir}\left(\mathbb{C}^{3}\right)_{\omega}\right)$ is a subgroup of $\operatorname{Bir}\left(\mathbb{C}^{3}\right)_{\mathrm{c}(\omega)}$ there is a natural left translation action of $\mathscr{K}$ (resp. $\left.\operatorname{Bir}\left(\mathbb{C}^{3}\right)_{\omega}\right)$ on $\operatorname{Bir}\left(\mathbb{C}^{3}\right)_{\mathrm{c}(\omega)}$. These two actions admit a complete invariant:

THEOREM 1.0.3. The map $\alpha$ is a complete invariant of the left translation action of $\mathscr{K}$ on $\operatorname{Bir}\left(\mathbb{C}^{3}\right)_{\mathrm{c}(\omega)}$, that is for any $\phi$ and $\psi$ in $\operatorname{Bir}\left(\mathbb{C}^{3}\right)_{\mathrm{c}(\omega)}$ one has $\alpha(\phi)=\alpha(\psi)$ if and only if $\psi \phi^{-1}$ belongs to $\mathscr{K}$.

The map $V$ is a complete invariant of the left translation action of $\operatorname{Bir}\left(\mathbb{C}^{3}\right)_{\omega}$ of $\operatorname{Bir}\left(\mathbb{C}^{3}\right)_{\mathrm{c}(\omega)}$, i.e. for any $\phi, \psi$ in $\operatorname{Bir}\left(\mathbb{C}^{3}\right)_{\mathrm{c}(\omega)}$ one has $V(\phi)=V(\psi)$ if and only if $\psi \phi^{-1}$ belongs to $\operatorname{Bir}\left(\mathbb{C}^{3}\right)_{\omega}$.

We prove that $\alpha$ is not surjective: generic linear differential equations of second order give linear functions that are not in the image of $\alpha$. Painlevé equations give examples of polynomials of higher degree that do not belong to $\operatorname{im} \alpha$. The map $V$ is also not surjective.

Since $\omega$ has no integral surface in $\mathbb{C}^{3}$ a contact birational self-map $\phi$ either preserves the hyperplane $z_{3}=0$, or blows down $z_{3}=0$. This naturally implies the following definition: $\phi \in \operatorname{Bir}\left(\mathbb{C}^{3}\right)_{\mathrm{c}(\omega)}$ is regular at infinity if $z_{3}=0$ is preserved by $\phi$ and if $\phi_{\mid z_{3}=0}$ is birational. One shows that

Proposition 1.0.4. The set of maps of $\operatorname{Bir}\left(\mathbb{C}^{3}\right)_{\omega}$ that are regular coincides with $\operatorname{Aut}\left(\mathbb{P}_{\mathbb{C}}^{3}\right)_{\omega}$

Let $\varsigma: \operatorname{Bir}\left(\mathbb{C}^{3}\right)_{\omega} \rightarrow \operatorname{Bir}\left(\mathbb{C}^{2}\right)_{\eta}$ be the projection onto the two first components. We say that $\varphi \in \operatorname{Bir}\left(\mathbb{C}^{2}\right)_{\eta}$ is exact if $\varphi$ can be lifted via $\varsigma$ to $\operatorname{Bir}\left(\mathbb{C}^{3}\right)_{\omega}$. One establishes the following criterion:

TheOREM 1.0.5. A map $\varphi=\left(\phi_{0}, \phi_{1}\right) \in \operatorname{Bir}\left(\mathbb{C}^{2}\right)_{\eta}$ is exact if and only if the closed 
form $\phi_{0} \mathrm{~d} \phi_{1}-z_{0} \mathrm{~d} z_{1}$ has trivial residues. In that case $\phi_{0} \mathrm{~d} \phi_{1}-z_{0} \mathrm{~d} z_{1}=-\mathrm{d} b$ with $b \in$ $\mathbb{C}\left(z_{0}, z_{1}\right)$ and $\phi=\left(\varphi, z_{2}+b\left(z_{0}, z_{1}\right)\right) \in \operatorname{Bir}\left(\mathbb{C}^{3}\right)_{\omega}$.

We give a lot of examples, and even subgroups, of exact maps but also prove that the map $\varsigma$ is not surjective:

THEOREM 1.0.6. A generic quadratic element of $\operatorname{Bir}\left(\mathbb{C}^{2}\right)_{\eta}$ is not exact.

Furthermore we look at invariant curves and surfaces. Thanks to a local argument of contact geometry one gets that if $\phi$ belongs to $\operatorname{Bir}\left(\mathbb{C}^{3}\right)_{\omega}$, if $m$ is a periodic point of $\phi$, and if there exists a germ of irreducible curve $\mathcal{C}$ invariant by $\phi$ and passing through $m$, then either $\mathcal{C}$ is a curve of periodic points, or $\mathcal{C}$ is a legendrian curve. We also give a precise description of elements of $\operatorname{Aut}\left(\mathbb{C}^{3}\right)_{\omega}\left(\right.$ resp. $\left.\operatorname{Bir}\left(\mathbb{C}^{3}\right)_{\omega}\right)$ that preserve a surface.

Besides we deal with some group properties. Danilov proved that $\operatorname{Aut}\left(\mathbb{C}^{2}\right)_{\eta}$ is not simple $([\mathbf{1 5}])$; Cantat and Lamy showed that $\operatorname{Bir}\left(\mathbb{P}_{\mathbb{C}}^{2}\right)$ is not simple $([\mathbf{1 1}])$. In the same spirit we establish that

Theorem 1.0.7. The groups $\operatorname{Aut}\left(\mathbb{C}^{3}\right)_{\omega}, \operatorname{Bir}\left(\mathbb{C}^{3}\right)_{\omega}, \operatorname{Aut}\left(\mathbb{C}^{3}\right)_{\mathrm{c}(\omega)}$, the derived group of $\operatorname{Aut}\left(\mathbb{C}^{3}\right)_{\omega}$ and the derived group of $\operatorname{Aut}\left(\mathbb{C}^{3}\right)_{\mathrm{c}(\omega)}$ are not simple.

Lamy proved that Aut $\left(\mathbb{C}^{2}\right)$ satisfies the Tits alternative $([\mathbf{2 6}])$, then Cantat showed that $\operatorname{Bir}\left(\mathbb{P}_{\mathbb{C}}^{2}\right)$ also $([\mathbf{1 0}])$. In our context one gets that

TheOREM 1.0.8. The groups $\operatorname{Aut}\left(\mathbb{C}^{3}\right)_{\omega}$, Aut $\left(\mathbb{C}^{3}\right)_{\mathrm{c}(\omega)}$ and $\operatorname{Bir}\left(\mathbb{C}^{3}\right)_{\omega}$ satisfy the Tits alternative.

Acknowledgements. We would like to thank Guy Casale for discussions about the non-integrability.

\section{Contact polynomial automorphisms.}

A polynomial automorphism $\phi$ of $\mathbb{C}^{n}$ is a polynomial map of the type

$$
\begin{gathered}
\phi: \mathbb{C}^{n} \rightarrow \mathbb{C}^{n}, \\
\left(z_{0}, z_{1}, \ldots, z_{n-1}\right) \mapsto\left(\phi_{0}\left(z_{0}, z_{1}, \ldots, z_{n-1}\right), \phi_{1}\left(z_{0}, z_{1}, \ldots, z_{n-1}\right), \ldots, \phi_{n-1}\left(z_{0}, z_{1}, \ldots, z_{n-1}\right)\right)
\end{gathered}
$$

that is bijective. The set of polynomial automorphisms of $\mathbb{C}^{n}$ form a group denoted $\operatorname{Aut}\left(\mathbb{C}^{n}\right)$.

The automorphisms of $\mathbb{C}^{n}$ of the form $\left(\phi_{0}, \phi_{1}, \ldots, \phi_{n-1}\right)$ where $\phi_{i}$ depends only on $z_{i}, z_{i+1}, \ldots, z_{n-1}$ form the Jonquières subgroup $\mathrm{J}_{n} \subset \operatorname{Aut}\left(\mathbb{C}^{n}\right)$. Moreover one has the inclusions

$$
G L\left(\mathbb{C}^{n}\right) \subset \operatorname{Aff}_{n} \subset \operatorname{Aut}\left(\mathbb{C}^{n}\right)
$$

where $\operatorname{Aff}_{n}$ denotes the group of affine maps

$$
\phi:\left(z_{0}, z_{1}, \ldots, z_{n-1}\right) \mapsto
$$




$$
\left(\phi_{0}\left(z_{0}, z_{1}, \ldots, z_{n-1}\right), \phi_{1}\left(z_{0}, z_{1}, \ldots, z_{n-1}\right), \ldots, \phi_{n-1}\left(z_{0}, z_{1}, \ldots, z_{n-1}\right)\right)
$$

with $\phi_{i}$ affine; Aff $n$ is the semi-direct product of $G L\left(\mathbb{C}^{n}\right)$ with the commutative subgroups of translations. The subgroup Tame ${ }_{n} \subset \operatorname{Aut}\left(\mathbb{C}^{n}\right)$ generated by $\mathrm{J}_{n}$ and $\mathrm{Aff}_{n}$ is called the group of tame automorphisms.

Convention. In all the article we denote $\mathbb{P}_{\mathbb{C}}^{n}$ by $\mathbb{P}^{n}$, and we write "birational maps of $\mathbb{P}^{n}$ " instead of "birational self-maps of $\mathbb{P}^{n}$ ".

\subsection{Contact forms and contact structures.}

We recall in the context of 3-manifolds the formalism of contact structure. Let $M$ be a complex 3-manifold; we denote by $\Omega^{i}(M)$ the space of holomorphic $i$-forms on $M$. A contact form on $M$ is an element $\Theta \in \Omega^{1}(M)$ such that the 3 -form $\Theta \wedge \mathrm{d} \Theta \in \Omega^{3}(M)$ has no zero: $\Theta \wedge \mathrm{d} \Theta(m) \neq 0$ for any $m \in M$. For such a contact form there is a local model given by Darboux theorem: at each point $m$ there is a local biholomorphism $F: M,_{m} \rightarrow \mathbb{C}^{3},_{0}$ such that $\Theta=F^{*}\left(z_{0} \mathrm{~d} z_{1}+\mathrm{d} z_{2}\right)$. The 1 -form $z_{0} \mathrm{~d} z_{1}+\mathrm{d} z_{2}$ is called the standard contact form on $\mathbb{C}^{3}$; we denote it by $\omega$.

A contact structure on the 3 -manifold $M$ is given by the following data:

(i) an open covering $M=\sqcup_{k} \mathcal{U}_{k}$,

(ii) on each $\mathcal{U}_{k}$ a contact form $\Theta_{k} \in \Omega^{1}\left(\mathcal{U}_{k}\right)$,

(iii) on each non-trivial intersection $\mathcal{U}_{k} \cap \mathcal{U}_{\ell}$ a holomorphic unit $g_{k \ell} \in \mathcal{O}^{*}\left(\mathcal{U}_{k} \cap \mathcal{U}_{\ell}\right)$ such that $\Theta_{k}=g_{k \ell} \Theta_{\ell}$.

A contact structure defines a holomorphic hyperplanes field $t: M \rightarrow \mathbb{P}(\mathrm{T} M)^{\vee}$ given for all $m \in \mathcal{U}_{k}$ by

$$
t(m)=\operatorname{ker} \Theta_{k}(m) .
$$

The compact Kähler manifolds having a contact structure are classified by Frantzen and Peternell theorem $([\mathbf{1 8}])$. On $\mathbb{P}^{3}$ there is no contact form because there is no nontrivial global form. Nevertheless there are contact structures; one of them is given in homogeneous coordinates by the 1 -form

$$
\widetilde{\vartheta}=z_{0} \mathrm{~d} z_{1}-z_{1} \mathrm{~d} z_{0}+z_{2} \mathrm{~d} z_{3}-z_{3} \mathrm{~d} z_{2}
$$

In that case we can take the standard covering by affine charts $\mathcal{U}_{k}=\left\{z_{k}=1\right\}$ and $\vartheta_{k}=\widetilde{\vartheta}_{\mid \mathcal{U}_{k}}$.

Proposition 2.1.1. Up to automorphisms of $\mathbb{P}^{3}$ there is only one contact structure on $\mathbb{P}^{3}$.

Proof. Remark that to a contact structure on $\mathbb{P}^{3}$ is associated a homogeneous 1-form $\beta$ on $\mathbb{C}^{4}$ such that $\mathcal{U}_{k}=\left\{z_{k}=1\right\}$ and $\Theta_{k}=\beta_{\mid \mathcal{U}_{k}}$ satisfies properties i., ii., iii.

Let $\beta$ be a contact structure on $\mathbb{P}^{3}$, and let $R=\sum_{i} z_{i} \partial / \partial z_{i}$ be the radial vector field. Since $i_{R} \beta=0$, to give $\beta$ is equivalent to give $\mathrm{d} \beta$. According to [24, Chapter 2, Proposition 2.1] one has $\operatorname{deg} \mathrm{d} \beta=0$; to give $\mathrm{d} \beta$ is thus equivalent to give an antisymmetric 
matrix of maximal rank. But up to conjugacy there is only one $4 \times 4$ antisymmetric matrix of maximal rank.

REMARK 2.1.2. The group of linear automorphisms of $\mathbb{C}^{4}$ that preserve $\widetilde{\vartheta}$ coincides with the group of automorphisms of $\mathbb{P}^{3}$ that preserve $\mathrm{d} \widetilde{\vartheta}$; as a consequence the subgroup of $\operatorname{Aut}\left(\mathbb{P}^{3}\right)$ that preserves the contact structure associated to $\mathrm{d} \widetilde{\vartheta}$ is the projectivization of the symplectic group $\operatorname{Sp}(4 ; \mathbb{C})$.

Remark that the data of a global meromorphic 1-form $\Theta$ on $M$ such that $\Theta \wedge \mathrm{d} \Theta \not \equiv 0$ induces a contact form (and a contact structure) on the complement of the poles and zeros of $\Theta$ and $\Theta \wedge \mathrm{d} \Theta$. In that case we say that $\Theta$ induces a meromorphic contact structure on $M$.

For instance the Darboux form $\omega=z_{0} \mathrm{~d} z_{1}+\mathrm{d} z_{2}$ induces a meromorphic contact structure on $\mathbb{P}^{3}$. In fact the forms $\omega$ and $\widetilde{\vartheta}_{\mid z_{3}=1}$ are conjugate on $\mathbb{C}^{3}$ via $\left(z_{0} / 2, z_{1},-z_{2}+\right.$ $\left.z_{0} z_{1} / 2\right)$. The corresponding (meromorphic) contact structure are birationally conjugate on $\mathbb{P}^{3}$.

\subsection{Description of contact automorphisms.}

Let us describe Aut $\left(\mathbb{C}^{3}\right)_{\omega}$. Set $\eta=\mathrm{d} \omega=\mathrm{d} z_{0} \wedge \mathrm{d} z_{1}$. Remark that the invariance of $\omega$ implies the invariance of $\eta$ and as a consequence the equality $\left(\phi_{0}, \phi_{1}\right)^{*} \eta=\eta$.

Proposition 2.2.1. If $\phi$ belongs to Aut $\left(\mathbb{C}^{3}\right)_{\omega}$, then $\phi_{*} \partial / \partial z_{2}=\partial / \partial z_{2}$. In particular if $\phi$ belongs to $\operatorname{Aut}\left(\mathbb{C}^{3}\right)_{\omega}$, then

$$
\phi=\left(\phi_{0}\left(z_{0}, z_{1}\right), \phi_{1}\left(z_{0}, z_{1}\right), z_{2}+b\left(z_{0}, z_{1}\right)\right)
$$

and the map

$$
\begin{gathered}
\varsigma: \operatorname{Aut}\left(\mathbb{C}^{3}\right)_{\omega} \longrightarrow \operatorname{Aut}\left(\mathbb{C}^{2}\right)_{\eta} \\
\left(\phi_{0}\left(z_{0}, z_{1}\right), \phi_{1}\left(z_{0}, z_{1}\right), z_{2}+b\left(z_{0}, z_{1}\right)\right) \mapsto\left(\phi_{0}\left(z_{0}, z_{1}\right), \phi_{1}\left(z_{0}, z_{1}\right)\right)
\end{gathered}
$$

is a morphism.

Proof. As we already mentioned, for a contact form there exists a unique vector field $\chi$, called Reeb vector field, such that $\omega(\chi)=1$ and $i_{\chi} \mathrm{d} \omega=0$; here $\chi=\partial / \partial z_{2}$. If $\phi$ belongs to $\operatorname{Aut}\left(\mathbb{C}^{3}\right)_{\omega}$, then $\phi_{*} \chi=\chi$. As a result $\phi$ has the following form

$$
\phi=\left(\phi_{0}\left(z_{0}, z_{1}\right), \phi_{1}\left(z_{0}, z_{1}\right), z_{2}+b\left(z_{0}, z_{1}\right)\right)
$$

with $\left(\phi_{0}, \phi_{1}\right)$ in $\operatorname{Aut}\left(\mathbb{C}^{2}\right)$ and $b$ in $\mathbb{C}\left[z_{0}, z_{1}\right]$.

REMARK 2.2.2. Any element of $\operatorname{Aut}\left(\mathbb{C}^{3}\right)_{\mathrm{c}(\omega)}$ can be written

$$
\left(\varphi_{0}, \varphi_{1}, \operatorname{det} \operatorname{jac} \varphi z_{2}+b\left(z_{0}, z_{1}\right)\right)
$$

where $\varphi=\left(\varphi_{0}, \varphi_{1}\right) \in \operatorname{Aut}\left(\mathbb{C}^{2}\right)$ and $\mathrm{d} b=(\operatorname{det} \operatorname{jac} \varphi) z_{0} \mathrm{~d} z_{1}-\varphi_{0} \mathrm{~d} \varphi_{1}$. Let us still denote by $\varsigma$ the natural projection 


$$
\varsigma: \operatorname{Aut}\left(\mathbb{C}^{3}\right)_{\mathrm{c}(\omega)} \rightarrow \operatorname{Aut}\left(\mathbb{C}^{2}\right)
$$

An element $\phi$ of $\operatorname{Bir}\left(\mathbb{C}^{2}\right)_{\eta}$ is exact if it can be lifted via $\varsigma$ to $\operatorname{Bir}\left(\mathbb{C}^{3}\right)_{\omega}$, or equivalently if it belongs to im $\varsigma$.

Contrary to the birational case (Theorem 3.4.1) any element of Aut $\left(\mathbb{C}^{2}\right)$ can be lifted via $\varsigma$ to $\operatorname{Aut}\left(\mathbb{C}^{3}\right)_{c(\omega)}$. Since $b$ is defined up to a constant we do not speak about the $\varsigma$-lift but a $\varsigma$-lift.

The following obvious statement describes the group $\operatorname{Aut}\left(\mathbb{C}^{3}\right)_{\omega}$ :

Proposition 2.2.3. Let us consider the morphism

$$
\begin{gathered}
\varsigma: \operatorname{Aut}\left(\mathbb{C}^{3}\right)_{\omega} \longrightarrow \operatorname{Aut}\left(\mathbb{C}^{2}\right)_{\eta} \\
\left(\phi_{0}\left(z_{0}, z_{1}\right), \phi_{1}\left(z_{0}, z_{1}\right), z_{2}+b\left(z_{0}, z_{1}\right)\right) \mapsto\left(\phi_{0}\left(z_{0}, z_{1}\right), \phi_{1}\left(z_{0}, z_{1}\right)\right) .
\end{gathered}
$$

One has the following exact sequence

$$
0 \longrightarrow \mathbb{C} \longrightarrow \operatorname{Aut}\left(\mathbb{C}^{3}\right)_{\omega} \stackrel{\varsigma}{\longrightarrow} \operatorname{Aut}\left(\mathbb{C}^{2}\right)_{\eta} \longrightarrow 1 ;
$$

more precisely $\operatorname{ker} \varsigma=\left\{\left(z_{0}, z_{1}, z_{2}+\beta\right) \mid \beta \in \mathbb{C}\right\}$. In particular

$$
\operatorname{Aut}\left(\mathbb{C}^{3}\right)_{\omega} \simeq \operatorname{Aut}\left(\mathbb{C}^{2}\right)_{\eta} \ltimes \mathbb{C} .
$$

Proof. The 1 -form $\phi_{0} \mathrm{~d} \phi_{1}-z_{0} \mathrm{~d} z_{1}$ is a closed and polynomial one, so it is exact. Therefore $\varsigma$ is surjective.

Let $\mathrm{G}$ be a group. The derived group of $\mathrm{G}$ is the subgroup of $\mathrm{G}$ generated by all the commutators of $\mathrm{G}$ :

$$
[\mathrm{G}, \mathrm{G}]=\left\langle g h g^{-1} h^{-1} \mid g, h \in \mathrm{G}\right\rangle .
$$

The group $\mathrm{G}$ is said to be perfect if it coincides with its derived group, or equivalently, if the group has no nontrivial abelian quotients.

Such a property was established in the context of real smooth manifolds: Banyaga proved that the derived group of the group of contact diffeomorphisms is a perfect one $([2][3][4])$.

TheOREM 2.2.4. The group $\left[\operatorname{Aut}\left(\mathbb{C}^{3}\right)_{\omega}, \operatorname{Aut}\left(\mathbb{C}^{3}\right)_{\omega}\right]$ is perfect.

Proof. Since $\varsigma$ is surjective (Proposition 2.2.3) and $\operatorname{Aut}\left(\mathbb{C}^{2}\right)_{\eta}$ is perfect $([\mathbf{2 0}$, Proposition 10]) the restriction of $\varsigma$

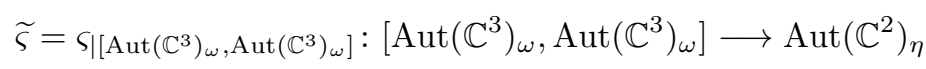

is surjective. Let $\phi$ be in $\operatorname{ker} \widetilde{\varsigma}$; on the one hand $\phi=\left(z_{0}, z_{1}, z_{2}+\beta\right)$ for some $\beta$ (Proposition 2.2.3), and on the other hand $\phi$ is a product of commutators hence $\beta=0$. We thus have the following exact sequence 


$$
0 \longrightarrow\left[\operatorname{Aut}\left(\mathbb{C}^{3}\right)_{\omega}, \operatorname{Aut}\left(\mathbb{C}^{3}\right)_{\omega}\right] \longrightarrow \operatorname{Aut}\left(\mathbb{C}^{2}\right)_{\eta} \longrightarrow 1
$$

and $\left[\operatorname{Aut}\left(\mathbb{C}^{3}\right)_{\omega}, \operatorname{Aut}\left(\mathbb{C}^{3}\right)_{\omega}\right] \simeq \operatorname{Aut}\left(\mathbb{C}^{2}\right)_{\eta}$ which is perfect $([\mathbf{2 0}$, Proposition 10]).

We will now describe $\operatorname{Aut}\left(\mathbb{C}^{3}\right)_{\mathrm{c}(\omega)}$. Let us recall that $\operatorname{Aut}\left(\mathbb{C}^{2}\right)$ is generated by $\mathrm{J}_{2}$ and $\mathrm{Aff}_{2}$ (see [25]). This implies that $\mathrm{Aff}_{2}$ and

$$
\left[\mathrm{J}_{2}, \mathrm{~J}_{2}\right]=\left\{\left(z_{0}+\beta, z_{1}+P\left(z_{0}\right)\right) \mid \beta \in \mathbb{C}, P \in \mathbb{C}\left[z_{0}\right]\right\} .
$$

generate $\operatorname{Aut}\left(\mathbb{C}^{2}\right)$.

Proposition 2.2.5. The group $\operatorname{Aut}\left(\mathbb{C}^{3}\right)_{\mathrm{c}(\omega)}$ is generated by $\mathcal{A}$ and $\mathcal{E}$ where

$$
\mathcal{E}=\left\{\varsigma \text {-lifts of } \mathfrak{e} \mid \mathfrak{e} \in\left[\mathrm{J}_{2}, \mathrm{~J}_{2}\right]\right\} \quad \text { and } \quad \mathcal{A}=\left\{\varsigma \text {-lifts of } \mathfrak{a} \mid \mathfrak{a} \in \mathrm{Aff}_{2}\right\} .
$$

Proof. Let $\varphi$ be a polynomial automorphism of $\mathbb{C}^{2}$ and let $\phi$ be a $\varsigma$-lift of $\varphi$ to $\operatorname{Aut}\left(\mathbb{C}^{3}\right)_{\mathrm{c}(\omega)}$

$$
\phi=\left(\varphi, \operatorname{det} \operatorname{jac} \varphi z_{2}+b\left(z_{0}, z_{1}\right)\right)
$$

with $b$ in $\mathbb{C}\left[z_{0}, z_{1}\right]$. One can write $\varphi$ as $\mathfrak{a}_{1} \mathfrak{e}_{1} \mathfrak{a}_{2} \mathfrak{e}_{2} \cdots \mathfrak{a}_{s} \mathfrak{e}_{s}$ where $\mathfrak{a}_{i}$ belongs to Aff 2 and $\mathfrak{e}_{i}$ to $\left[\mathrm{J}_{2}, \mathrm{~J}_{2}\right]$. Let us now consider $A_{i}$ a $\varsigma$-lift of $\mathfrak{a}_{i}, E_{i}=\left(\mathfrak{e}_{i}, z_{2}+d_{i}\right)$ a $\varsigma$-lift of $\mathfrak{e}_{i}$. Then $A_{1} E_{1} A_{2} E_{2} \cdots A_{s} E_{s}$ belongs to $\operatorname{Aut}\left(\mathbb{C}^{3}\right)_{\mathrm{c}(\omega)}$, and up to composition by an element $\left(z_{0}, z_{1}, z_{2}+\beta\right) \in \mathcal{A}$ one has

$$
\phi=A_{1} E_{1} A_{2} E_{2} \cdots A_{s} E_{s} .
$$

Proposition 2.2.6. One has

$$
\operatorname{Aut}\left(\mathbb{C}^{3}\right)_{\mathrm{c}(\omega)} \simeq \operatorname{Aut}\left(\mathbb{C}^{3}\right)_{\omega} \ltimes \mathbb{C}^{*}
$$

Proof. Let us consider an element $\phi$ of $\operatorname{Aut}\left(\mathbb{C}^{3}\right)_{\mathrm{c}(\omega)}$, then $\phi^{*} \omega=V(\phi) \omega$ for some polynomial $V(\phi)$. As $\omega$ and $\phi^{*} \omega$ do not vanish, $V(\phi)$ does not vanish; therefore $V(\phi)=\lambda \in \mathbb{C}^{*}$. Let us write $\phi$ as follows:

$$
\phi=\left(\lambda z_{0}, z_{1}, \lambda z_{2}\right) \circ \widetilde{\phi}
$$

of course $\widetilde{\phi}^{*} \omega=\omega$.

TheORem 2.2.7. The derived group $\left[\operatorname{Aut}\left(\mathbb{C}^{3}\right)_{\mathrm{c}(\omega)}, \operatorname{Aut}\left(\mathbb{C}^{3}\right)_{\mathrm{c}(\omega)}\right]$ of $\operatorname{Aut}\left(\mathbb{C}^{3}\right)_{\mathrm{c}(\omega)}$ is perfect.

Proof. According to Proposition 2.2.6 an element $\phi$ of $\operatorname{Aut}\left(\mathbb{C}^{3}\right)_{c(\omega)}$ can be written

$$
\left(\lambda \phi_{0}, \phi_{1}, \lambda z_{2}+\lambda b\right)
$$

with $\lambda \in \mathbb{C}^{*}$ and $\left(\phi_{0}, \phi_{1}, z_{2}+b\right) \in \operatorname{Aut}\left(\mathbb{C}^{3}\right)_{\omega}$. Denote by $\varphi$ the element of $\operatorname{Aut}\left(\mathbb{C}^{2}\right)$ given by $\left(\phi_{0}, \phi_{1}\right)$. If $\phi$ belongs to $\operatorname{ker} \varsigma$, then $\lambda=1, \varphi=\mathrm{id}$ and $b \in \mathbb{C}$, that is $\operatorname{ker} \varsigma \simeq \mathbb{C}$ and 


$$
\mathbb{C} \longrightarrow \operatorname{Aut}\left(\mathbb{C}^{3}\right)_{\mathrm{c}(\omega)} \stackrel{\varsigma}{\longrightarrow} \operatorname{Aut}\left(\mathbb{C}^{2}\right) \longrightarrow 1 .
$$

Since Aut $\left(\mathbb{C}^{2}\right)_{\eta}$ is perfect the restriction of $\varsigma$ to $\left[\operatorname{Aut}\left(\mathbb{C}^{3}\right)_{c(\omega)}, \operatorname{Aut}\left(\mathbb{C}^{3}\right)_{\mathrm{c}(\omega)}\right]$ induces the following exact sequence

$$
0 \longrightarrow\left[\operatorname{Aut}\left(\mathbb{C}^{3}\right)_{\mathrm{c}(\omega)}, \operatorname{Aut}\left(\mathbb{C}^{3}\right)_{\mathrm{c}(\omega)}\right] \longrightarrow \operatorname{Aut}\left(\mathbb{C}^{2}\right)_{\eta} \longrightarrow 1
$$

and $\left[\operatorname{Aut}\left(\mathbb{C}^{3}\right)_{\mathrm{c}(\omega)}, \operatorname{Aut}\left(\mathbb{C}^{3}\right)_{\mathrm{c}(\omega)}\right] \simeq \operatorname{Aut}\left(\mathbb{C}^{2}\right)_{\eta}$. One concludes as previously with $[\mathbf{2 0}$, Proposition 10].

Let us now deal with the finite subgroups of $\operatorname{Aut}\left(\mathbb{C}^{3}\right)_{\mathrm{c}(\omega)}$.

Proposition 2.2.8. Any element of Aut $\left(\mathbb{C}^{2}\right)_{\eta}$ of period $\ell$ lifts via $\varsigma$ to a unique element of $\operatorname{Aut}\left(\mathbb{C}^{3}\right)_{\omega}$ of period $\ell$.

Proof. Let us consider an element $\varphi=\left(\phi_{0}\left(z_{0}, z_{1}\right), \phi_{1}\left(z_{0}, z_{1}\right)\right)$ of $\operatorname{Aut}\left(\mathbb{C}^{2}\right)_{\eta}$. According to Proposition 2.2.3 there exists $b \in \mathbb{C}\left[z_{0}, z_{1}\right]$ such that $\left(\phi_{0}\left(z_{0}, z_{1}\right), \phi_{1}\left(z_{0}, z_{1}\right), z_{2}+\right.$ $\left.b\left(z_{0}, z_{1}\right)+\mu\right)$ belongs to $\operatorname{Bir}\left(\mathbb{C}^{3}\right)_{\omega}$ for any $\mu \in \mathbb{C}$. Assume that $\varphi$ is of prime order $\ell$; let us prove that there exists a unique $\gamma \in \mathbb{C}$ such that

$$
\left(\phi_{0}, \phi_{1}, z_{2}+b\left(z_{0}, z_{1}\right)+\gamma\right)
$$

is of order $\ell$.

Assume for simplicity that $\ell=2$ (but a similar argument works for any $\ell$ ). Let us recall that the following equality holds

$$
z_{0} \mathrm{~d} z_{1}-\phi_{0} \mathrm{~d} \phi_{1}=\mathrm{d} b .
$$

Applying $\phi$ to this equality one gets

$$
\phi_{0} \mathrm{~d} \phi_{1}-z_{0} \mathrm{~d} z_{1}=\mathrm{d}(b \circ \varphi) .
$$

We add (2.3) and (2.4) and obtain that $b+b \circ \phi$ is a constant $\beta$. Furthermore

$\left(\phi_{0}\left(z_{0}, z_{1}\right), \phi_{1}\left(z_{0}, z_{1}\right), z_{2}+b\left(z_{0}, z_{1}\right)+\mu\right)^{2}=\left(z_{0}, z_{1}, z_{2}+2 \gamma+b+b \circ \varphi\right)=\left(z_{0}, z_{1}, z_{2}+2 \gamma+\beta\right)$

so as soon as $\gamma=-\beta / 2$ one has $\left(\phi_{0}\left(z_{0}, z_{1}\right), \phi_{1}\left(z_{0}, z_{1}\right), z_{2}+b\left(z_{0}, z_{1}\right)+\mu\right)^{2}=$ id.

Proposition 2.2.9. A finite subgroup of $\operatorname{Aut}\left(\mathbb{C}^{2}\right)$ can be lifted to a finite subgroup of $\operatorname{Aut}\left(\mathbb{C}^{3}\right) \mathrm{c}(\omega)$.

Proof. $\quad$ Let $\mathrm{H}$ be a finite subgroup of $\operatorname{Aut}\left(\mathbb{C}^{2}\right)$. The group $\mathrm{H}$ is linearizable $([\mathbf{2 2}])$ hence has a fixed point $p$. Since the translations belong to Aut $\left(\mathbb{C}^{2}\right)$ one can assume that $p=(0,0)$. Let us consider the lifts of all elements of $\mathrm{H}$ in $\left\{\phi \in \operatorname{Aut}\left(\mathbb{C}^{3}\right)_{\mathrm{c}(\omega)} \mid \phi(0)=0\right\}$; they form a group isomorphic to $\mathrm{H}$ so is in particular finite.

REMARK 2.2.10. Any subgroup G of Aut $\left(\mathbb{C}^{2}\right)$ that preserves $(0,0)$ can be lifted to a subgroup of $\operatorname{Aut}\left(\mathbb{C}^{3}\right)_{\mathrm{c}(\omega)}$ isomorphic to $\mathrm{G}$. 
TheOREM 2.2.11. Any finite subgroup of $\operatorname{Aut}\left(\mathbb{C}^{3}\right)_{\mathrm{c}(\omega)}$ is linearizable via an element of $\operatorname{Aut}\left(\mathbb{C}^{3}\right)_{\mathrm{c}(\omega)}$.

Proof. Let $\mathrm{G}$ be a finite subgroup of $\operatorname{Aut}\left(\mathbb{C}^{3}\right)_{\mathrm{c}(\omega)}$. The group $\mathrm{G}$ is isomorphic to $\mathrm{H}=\varsigma(\mathrm{G})$ which is thus a finite subgroup of $\operatorname{Aut}\left(\mathbb{C}^{2}\right)$. There exists a map $h \in \operatorname{Aut}\left(\mathbb{C}^{2}\right)$ that linearizes $\mathrm{H}$ (see $[\mathbf{2 2}])$; as a result $\mathrm{H}$ has a fixed point $p$ and up to translations one can suppose that $p=(0,0)$. Note that $h(0)=0$. The lift of $h$ in $\left\{\phi \in \operatorname{Aut}\left(\mathbb{C}^{3}\right)_{\mathrm{c}(\omega)} \mid \phi(0)=0\right\}$ linearizes $\mathrm{G}$.

\subsection{Automorphisms group.}

Let us first introduce some notations. The group of the field automorphisms of $\mathbb{C}$ acts on $\operatorname{Aut}\left(\mathbb{C}^{n}\right)\left(\right.$ resp. $\left.\operatorname{Bir}\left(\mathbb{P}^{n}\right)\right)$ : if $f$ is an element of $\operatorname{Aut}\left(\mathbb{C}^{n}\right)$ and if $\xi$ is a field automorphism we denote by ${ }^{\xi} f$ the element obtained by letting $\xi$ acting on $f$. Using the structure of amalgamated product of $\operatorname{Aut}\left(\mathbb{C}^{2}\right)$, the automorphisms of this group have been described $([\mathbf{1 6}])$ : let $\varphi$ be an automorphism of $\operatorname{Aut}\left(\mathbb{C}^{2}\right)$; there exist a polynomial automorphism $\psi$ of $\mathbb{C}^{2}$ and a field automorphism $\xi$ such that

$$
\forall f \in \operatorname{Aut}\left(\mathbb{C}^{2}\right), \quad \varphi(f)={ }^{\xi}\left(\psi f \psi^{-1}\right) .
$$

Even if $\operatorname{Bir}\left(\mathbb{P}^{2}\right)$ has not the same structure as $\operatorname{Aut}\left(\mathbb{C}^{2}\right)$ (see Appendix of $[\mathbf{1 1}]$ ) the automorphisms group of $\operatorname{Bir}\left(\mathbb{P}^{2}\right)$ can be described and a similar result is obtained $([\mathbf{1 7}])$.

We now would like to describe the group $\operatorname{Aut}\left(\operatorname{Aut}\left(\mathbb{C}^{3}\right)_{\omega}\right)$. Let us recall that the center of a group $\mathrm{G}$, denoted $Z(\mathrm{G})$, is the set of elements that commute with every element of G.

Proposition 2.3.1. The center of $\operatorname{Aut}\left(\mathbb{C}^{3}\right)_{\omega}$ is isomorphic to $\mathbb{C}$ :

$$
Z\left(\operatorname{Aut}\left(\mathbb{C}^{3}\right)_{\omega}\right)=\left\{\left(z_{0}, z_{1}, z_{2}+\beta\right) \mid \beta \in \mathbb{C}\right\}
$$

and the center of $\operatorname{Aut}\left(\mathbb{C}^{3}\right)_{\mathrm{c}(\omega)}$ is trivial.

As $\operatorname{Aut}\left(\mathbb{C}^{3}\right)_{\omega} \simeq \operatorname{Aut}\left(\mathbb{C}^{2}\right)_{\eta} \ltimes \mathbb{C}$ Proposition 2.3.1 implies the following statement:

Corollary 2.3.2. The quotient of $\operatorname{Aut}\left(\mathbb{C}^{3}\right)_{\omega}$ by its center is isomorphic to $\operatorname{Aut}\left(\mathbb{C}^{2}\right)_{\eta}$.

LEMMA 2.3.3. One has the following isomorphism

$$
\operatorname{Hom}\left(\operatorname{Aut}\left(\mathbb{C}^{3}\right)_{\omega}, \mathbb{C}\right) \simeq \operatorname{Hom}(\mathbb{C}, \mathbb{C})
$$

where $\operatorname{Hom}(\mathbb{C}, \mathbb{C})$ denotes the homomorphisms of the additive group $\mathbb{C}$.

Proof. Note that if $\phi$ belongs to $\left[\operatorname{Aut}\left(\mathbb{C}^{3}\right)_{\omega}, \operatorname{Aut}\left(\mathbb{C}^{3}\right)_{\omega}\right]$, then the last component of $\phi$ is well defined (that is not defined modulo a constant). Besides $\operatorname{Aut}\left(\mathbb{C}^{3}\right)_{\omega} \simeq$ $\operatorname{Aut}\left(\mathbb{C}^{2}\right)_{\eta} \ltimes \mathbb{C}$ and $\operatorname{Aut}\left(\mathbb{C}^{2}\right)_{\eta}$ is perfect thus

$$
\operatorname{Aut}\left(\mathbb{C}^{3}\right)_{\omega} /\left[\operatorname{Aut}\left(\mathbb{C}^{3}\right)_{\omega}, \operatorname{Aut}\left(\mathbb{C}^{3}\right)_{\omega}\right] \simeq \mathbb{C}
$$


and

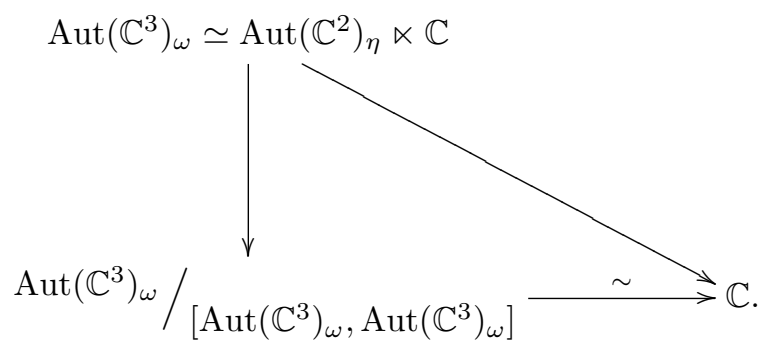

We conclude by noting that any element of $\operatorname{Hom}\left(\operatorname{Aut}\left(\mathbb{C}^{3}\right)_{\omega}, \mathbb{C}\right)$ acts trivially on $\phi$.

REMARK 2.3.4. An element $c$ of $\operatorname{Hom}\left(\operatorname{Aut}\left(\mathbb{C}^{3}\right)_{\omega}, \mathbb{C}\right)$ acts on $\operatorname{Aut}\left(\mathbb{C}^{3}\right)_{\omega}$ as follows

$$
\left(\phi_{0}, \phi_{1}, z_{2}+b\left(z_{0}, z_{1}\right)\right) \rightarrow\left(\phi_{0}, \phi_{1}, z_{2}+b\left(z_{0}, z_{1}\right)+c(\phi)\right) .
$$

Definition. Let $\mathrm{H}$ be a normal subgroup of a group $\mathrm{G}$. We say that an automorphism of $\mathrm{H}$ of the form $\phi \mapsto \varphi \phi \varphi^{-1}$, with $\varphi$ in $\mathrm{G}$, is $\mathrm{G}$-inner.

TheOREM 2.3.5. The group $\operatorname{Aut}\left(\operatorname{Aut}\left(\mathbb{C}^{3}\right)_{\omega}\right)$ is generated by the automorphisms group of the field $\mathbb{C}$, the group of $\operatorname{Aut}\left(\mathbb{C}^{3}\right)_{\mathrm{c}(\omega)}$-inner automorphisms and the action of $\operatorname{Hom}(\mathbb{C}, \mathbb{C})$.

Proof. Consider an element $\psi$ of $\operatorname{Aut}\left(\operatorname{Aut}\left(\mathbb{C}^{3}\right)_{\omega}\right)$. For any $\phi=\left(\varphi_{\phi}, z_{2}+\right.$ $\left.T_{\phi}\left(z_{0}, z_{1}\right)\right)$ one has

$$
\psi(\phi)=\left(\widetilde{\varphi_{\phi}}, z_{2}+\Delta_{\phi}\left(z_{0}, z_{1}\right)\right)
$$

In particular $\psi$ induces an automorphism $\psi_{0}$ of $\operatorname{Aut}\left(\mathbb{C}^{2}\right)_{\eta}$; indeed since $\psi$ is an automorphism of $\operatorname{Aut}\left(\mathbb{C}^{3}\right)_{\omega}$, it preserves $Z\left(\operatorname{Aut}\left(\mathbb{C}^{3}\right)_{\omega}\right)$ and so, from Corollary 2.3.2 induces an automorphism of $\operatorname{Aut}\left(\mathbb{C}^{2}\right)_{\eta}$.

According to Theorem 5.0.2 one can assume that $\psi_{0}=$ id up to the action of an automorphism of the field $\mathbb{C}$ and up to conjugacy by an Aut $\left(\mathbb{C}^{2}\right)$-inner automorphism, i.e.

$$
\psi(\phi)=\left(\varphi_{\phi}, z_{2}+\Delta_{\phi}\left(z_{0}, z_{1}\right)\right) .
$$

Set $\phi^{-1}=\left(\varphi_{\phi}^{-1}, z_{2}+T_{\phi^{-1}}\left(z_{0}, z_{1}\right)\right)$. On the one hand $\phi^{-1} \circ \phi=\left(\mathrm{id}, z_{2}+T_{\phi}\left(z_{0}, z_{1}\right)+\right.$ $\left.T_{\phi^{-1}}\left(\varphi_{\phi}\right)\right)$ so

$$
T_{\phi}+T_{\phi^{-1}}\left(\varphi_{\phi}\right)=0
$$

and on the other hand

$$
\psi\left(\phi \circ \phi^{-1}\right)=\left(\mathrm{id}, z_{2}+T_{\phi^{-1}}\left(z_{0}, z_{1}\right)+\Delta_{\phi} \varphi_{\phi}^{-1}\right)
$$

belongs to $\operatorname{Aut}\left(\mathbb{C}^{3}\right)_{\omega}$ hence $T_{\phi^{-1}}+\Delta_{\phi} \varphi_{\phi}^{-1}$ is a constant. This, combined with (2.5), 
implies that $\Delta_{\phi}=T_{\phi}+c_{\phi}$, where $c_{\phi}$ is a constant, and yields to a morphism from $\operatorname{Aut}\left(\mathbb{C}^{3}\right)_{\omega}$ to $\mathbb{C}$ :

$$
\operatorname{Aut}\left(\mathbb{C}^{3}\right)_{\omega} \rightarrow \mathbb{C}, \quad \phi \mapsto c_{\phi}
$$

Consider an homomorphism

$$
\rho: \operatorname{Aut}\left(\mathbb{C}^{3}\right)_{\omega} \rightarrow \mathbb{C}, \quad \phi \mapsto \rho_{\phi} .
$$

Let us define $\psi: \operatorname{Aut}\left(\mathbb{C}^{3}\right)_{\omega} \rightarrow \operatorname{Aut}\left(\mathbb{C}^{3}\right)_{\omega}$ by:

$$
\psi(\phi)=\left(\phi_{0}\left(z_{0}, z_{1}\right), \phi_{1}\left(z_{0}, z_{1}\right), z_{2}+b\left(z_{0}, z_{1}\right)+\rho_{\phi}\right)
$$

where $\phi=\left(\phi_{0}\left(z_{0}, z_{1}\right), \phi_{1}\left(z_{0}, z_{1}\right), z_{2}+b\left(z_{0}, z_{1}\right)\right) \in \operatorname{Aut}\left(\mathbb{C}^{3}\right)_{\omega}$. One can check that $\psi$ belongs to $\operatorname{Aut}\left(\operatorname{Aut}\left(\mathbb{C}^{3}\right)_{\omega}\right)$.

\section{Contact birational maps.}

A rational map of $\mathbb{P}^{n}$ can be written

$$
\begin{gathered}
\phi: \mathbb{P}^{n} \rightarrow \mathbb{P}^{n} \\
\left(z_{0}: z_{1}: \cdots: z_{n}\right) \rightarrow\left(\phi_{0}\left(z_{0}, z_{1}, \ldots, z_{n}\right): \phi_{1}\left(z_{0}, z_{1}, \ldots, z_{n}\right): \cdots: \phi_{n}\left(z_{0}, z_{1}, \ldots, z_{n}\right)\right)
\end{gathered}
$$

where the $\phi_{i}$ 's are homogeneous polynomials of the same degree $\geq 1$ and without common factor of positive degree. The degree of $\phi$ is by definition the degree of the $\phi_{i}$. A birational map of $\mathbb{P}^{n}$ is a rational map that admits a rational inverse. Of course $\operatorname{Aut}\left(\mathbb{C}^{n}\right)$ is a subgroup of $\operatorname{Bir}\left(\mathbb{P}^{n}\right)$. An other natural subgroup of $\operatorname{Bir}\left(\mathbb{P}^{n}\right)$ is the $\operatorname{group} \operatorname{Aut}\left(\mathbb{P}^{n}\right) \simeq$ $P G L(n+1 ; \mathbb{C})$ of automorphisms of $\mathbb{P}^{n}$.

The indeterminacy set Ind $\phi$ of $\phi$ is the set of the common zeros of the $\phi_{i}$ 's. The exceptional set $\operatorname{Exc} \phi$ of $\phi$ is the (finite) union of subvarieties $M_{i}$ of $\mathbb{P}^{n}$ such that $\phi$ is not injective on any open subset of $M_{i}$.

Let us extend the definition of Jonquières group we gave in the case of polynomial automorphisms of $\mathbb{C}^{n}$ to the case of birational maps of $\mathbb{P}^{2}$ : the Jonquières group, denoted $\mathcal{J}$, is the group of birational maps of $\mathbb{P}^{2}$ that preserve a pencil of rational curves. Since two pencils of rational curves are birationally conjugate, $\mathcal{J}$ does not depend, up to conjugacy, of the choice of the pencil. In other words one can decide, up to birational conjugacy, that $\mathcal{J}$ is in the affine chart $z_{2}=1$ the maximal group of birational maps that preserve the fibration $z_{1}=$ cst. An element $\varphi$ of $\mathcal{J}$ permutes the fibers of the fibration thus induces an automorphism of the base $\mathbb{P}^{1}$; note that if the fibration is fiberwise invariant, $\varphi$ acts as an homography in the generic fibers. Hence $\mathcal{J}$ can be identified with the semi-direct product $P G L\left(2 ; \mathbb{C}\left(z_{1}\right)\right) \rtimes P G L(2 ; \mathbb{C})$.

We study the birational maps $\phi=\left(\phi_{0}, \phi_{1}, \phi_{2}\right)$ defined on $\mathbb{C}^{3}=\left(z_{3}=1\right) \subset \mathbb{P}^{3}$ that preserve either the contact standard form $\omega$, or the contact structure $\mathrm{c}(\omega)$ associated to $\omega$. In other words we would like to describe the groups $\operatorname{Bir}\left(\mathbb{C}^{3}\right)_{\omega}$ and $\operatorname{Bir}\left(\mathbb{C}^{3}\right)_{c}(\omega)$ and also their elements.

Let us now illustrate a fundamental difference between $\operatorname{Bir}\left(\mathbb{C}^{3}\right)_{\omega}$ and $\operatorname{Bir}\left(\mathbb{C}^{3}\right)_{\mathrm{c}(\omega)}$ : 
the first group preserves the fibration associated to $\partial / \partial z_{2}$ whereas the second doesn't.

Proposition 3.0.6. If $\phi$ belongs to $\operatorname{Bir}\left(\mathbb{C}^{3}\right)_{\omega}$, then $\phi_{*} \partial / \partial z_{2}=\partial / \partial z_{2}$. In particular if $\phi$ belongs to $\operatorname{Bir}\left(\mathbb{C}^{3}\right)_{\omega}$, then

$$
\phi=\left(\phi_{0}\left(z_{0}, z_{1}\right), \phi_{1}\left(z_{0}, z_{1}\right), z_{2}+b\left(z_{0}, z_{1}\right)\right)
$$

and the map

$$
\begin{gathered}
\varsigma: \operatorname{Bir}\left(\mathbb{C}^{3}\right)_{\omega} \longrightarrow \operatorname{Bir}\left(\mathbb{C}^{2}\right)_{\eta} \\
\left(\phi_{0}\left(z_{0}, z_{1}\right), \phi_{1}\left(z_{0}, z_{1}\right), z_{2}+b\left(z_{0}, z_{1}\right)\right) \mapsto\left(\phi_{0}\left(z_{0}, z_{1}\right), \phi_{1}\left(z_{0}, z_{1}\right)\right)
\end{gathered}
$$

is a morphism.

REMARK 3.0.7. The proof is similar to the proof of Proposition 2.2.1.

REMARK 3.0.8. The first assertion of Proposition 3.0.6 is not true for the group $\operatorname{Bir}\left(\mathbb{C}^{3}\right)_{\mathrm{c}(\omega)}$; indeed let us consider the map $\psi$ defined by

$$
\psi=\left(\frac{z_{0}}{\left(1+z_{2}\right)^{2}}, z_{1}, \frac{z_{2}}{1+z_{2}}\right)
$$

it belongs to $\operatorname{Bir}\left(\mathbb{C}^{3}\right)_{\mathrm{c}(\omega)}$ and does not preserve the fibration associated to the vector field $\partial / \partial z_{2}$.

\subsection{A PDE approach.}

Let $\phi=\left(\phi_{0}, \phi_{1}, \phi_{2}\right)$ be in $\operatorname{Bir}\left(\mathbb{C}^{3}\right)_{\mathrm{c}(\omega)}$; then $\phi^{*} \omega=V(\phi) \omega$ for some rational function $V(\phi)$. One inherits a map $V$ from $\operatorname{Bir}\left(\mathbb{C}^{3}\right)_{\mathrm{c}(\omega)}$ into the set of rational functions in $z_{0}, z_{1}$ and $z_{2}$. The equality $\phi^{*} \omega=V(\phi) \omega$ gives the following system $(\star)$ of PDE:

$$
\left\{\begin{array}{l}
\phi_{0} \frac{\partial \phi_{1}}{\partial z_{0}}+\frac{\partial \phi_{2}}{\partial z_{0}}=0 \\
\phi_{0} \frac{\partial \phi_{1}}{\partial z_{1}}+\frac{\partial \phi_{2}}{\partial z_{1}}=V(\phi) z_{0} \\
\phi_{0} \frac{\partial \phi_{1}}{\partial z_{2}}+\frac{\partial \phi_{2}}{\partial z_{2}}=V(\phi)
\end{array}\right.
$$

Thanks to $\left(\star_{2}\right)$ and $\left(\star_{3}\right)$ one gets

$$
\phi_{0}\left(\frac{\partial \phi_{1}}{\partial z_{1}}-z_{0} \frac{\partial \phi_{1}}{\partial z_{2}}\right)+\left(\frac{\partial \phi_{2}}{\partial z_{1}}-z_{0} \frac{\partial \phi_{2}}{\partial z_{2}}\right)=0 .
$$

Equation $\left(\star_{1}\right)$ has a special family of solutions: maps for which both $\phi_{1}$ or $\phi_{2}$ do not depend on $z_{0}$ (note that if $\phi_{1}$ (resp. $\phi_{2}$ ) does not depend on $z_{0}$ then $\left(\star_{1}\right)$ implies that $\phi_{2}$ (resp. $\left.\phi_{1}\right)$ also); in that case we can then compute $\phi_{0}$ thanks to $\left(\star_{4}\right)$. Taking $\left(\phi_{1}, \phi_{2}\right)$ in $\operatorname{Bir}\left(\mathbb{P}^{2}\right)$ we get elements in $\operatorname{im} \mathcal{K}$; we will called this family of solutions Klein family. Note that this family is a group denoted $\mathscr{K}$, the Klein group.

Proposition 3.1.1. The elements of $\mathscr{K}$ are of the following type 


$$
\left(\frac{-\partial \phi_{2} / \partial z_{1}+z_{0} \partial \phi_{2} / \partial z_{2}}{\partial \phi_{1} / \partial z_{1}-z_{0} \partial \phi_{1} / \partial z_{2}}, \phi_{1}\left(z_{1}, z_{2}\right), \phi_{2}\left(z_{1}, z_{2}\right)\right)
$$

with $\left(\phi_{1}, \phi_{2}\right)$ in $\operatorname{Bir}\left(\mathbb{P}^{2}\right)$.

Assume now that $\phi_{1}$ or $\phi_{2}$ really depends on $z_{0}$ (i.e. that $\phi$ does not belong to the Klein family). Then $\left(\star_{1}\right)$ and $\left(\star_{4}\right)$ imply

$$
\left.\left(\frac{\partial \phi_{2}}{\partial z_{1}}-z_{0} \frac{\partial \phi_{2}}{\partial z_{2}}\right) \frac{\partial \phi_{1}}{\partial z_{0}}=\left(\frac{\partial \phi_{1}}{\partial z_{1}}-z_{0} \frac{\partial \phi_{1}}{\partial z_{2}}\right) \frac{\partial \phi_{2}}{\partial z_{0}} . \quad \quad \quad \quad \star_{5}\right)
$$

One can rewrite $\left(\star_{5}\right)$ as

$$
\frac{\partial \phi_{2} / \partial z_{1}-z_{0} \partial \phi_{2} / \partial z_{2}}{\partial \phi_{2} / \partial z_{0}}=\frac{\partial \phi_{1} / \partial z_{1}-z_{0} \partial \phi_{1} / \partial z_{2}}{\partial \phi_{1} / \partial z_{0}} .
$$

Denote by $\alpha$ the map from $\operatorname{Bir}\left(\mathbb{C}^{3}\right)_{\mathrm{c}(\omega)}$ to the set of rational functions in $z_{0}, z_{1}$ and $z_{2}$ defined by $\alpha(\phi)=\infty$ if $\phi$ belongs to $\mathscr{K}$ and

$$
\alpha(\phi)=\frac{\partial \phi_{2} / \partial z_{1}-z_{0} \partial \phi_{2} / \partial z_{2}}{\partial \phi_{2} / \partial z_{0}}=\frac{\partial \phi_{1} / \partial z_{1}-z_{0} \partial \phi_{1} / \partial z_{2}}{\partial \phi_{1} / \partial z_{0}}
$$

otherwise.

If $\phi_{1}$ and $\phi_{2}$ are some first integrals of

$$
Z_{\phi}=\alpha(\phi) \frac{\partial}{\partial z_{0}}-\frac{\partial}{\partial z_{1}}+z_{0} \frac{\partial}{\partial z_{2}}
$$

then $\left(\star_{5}\right)$ is satisfied. One thus gets $\phi_{0}$ from $\left(\star_{1}\right)$. Note that such a $\phi$ is not always birational. But one can get a lot of birational examples in this way.

For instance when $\alpha(\phi) \equiv 0$ one obtains a family of rational maps solutions of $(\star)$ and Legendre involution is one of them. The set of birational maps of that family is called Legendre family, i.e. it is the set of birational maps of the following form

$$
\left(-\frac{\left(\partial / \partial z_{0}\right)\left(\phi_{2}\left(z_{0},-\left(z_{2}+z_{0} z_{1}\right)\right)\right)}{\left(\partial / \partial z_{0}\right)\left(\phi_{1}\left(z_{0},-\left(z_{2}+z_{0} z_{1}\right)\right)\right)}, \phi_{1}\left(z_{0},-\left(z_{2}+z_{0} z_{1}\right)\right), \phi_{2}\left(z_{0},-\left(z_{2}+z_{0} z_{1}\right)\right)\right) .
$$

REMARK 3.1.2. The Legendre family composed with the Legendre involution (right composition) yields to the Klein family.

Definition. Let $\gamma$ be an irreducible curve; $\gamma$ is a legendrian curve if $s_{\gamma}^{*} \omega=0$ where $s_{\gamma}$ denotes a local parametrization of $\gamma$.

REMARK 3.1.3. Elements of the Klein family preserve the fibration $\left\{z_{1}=\right.$ cst, $z_{2}=$ cst $\}$; note that its fibers are legendrian curves. The Legendre involution sends the fibration $\left\{z_{0}=\right.$ cst, $z_{2}+z_{0} z_{1}=$ cst $\}$ onto $\left\{z_{1}=\right.$ cst, $z_{2}=$ cst $\}$. Then of course if one conjugates the Klein family by the Legendre involution one gets a family that preserves the fibration by legendrian curves $\left\{z_{0}=\right.$ cst, $z_{2}+z_{0} z_{1}=$ cst $\}$. 
A direct computation implies:

Proposition 3.1.4. Let $\phi=\left(\phi_{0}, \phi_{1}, \phi_{2}\right)$ be a contact birational map of $\mathbb{P}^{3}$. The map $\phi$ conjugates the foliation induced by $Z_{\phi}$ to the foliation induced by $\partial / \partial z_{0}$. As a consequence the field of the rational first integrals of $Z_{\phi}$ is generated by $\phi_{1}$ and $\phi_{2}$.

The left translation action of $\mathscr{K}$ on $\operatorname{Bir}\left(\mathbb{C}^{3}\right)_{c}(\omega)$ is given by

$$
(\psi, \phi) \in \mathscr{K} \times \operatorname{Bir}\left(\mathbb{C}^{3}\right)_{\mathrm{c}(\omega)} \longrightarrow \psi \phi \in \operatorname{Bir}\left(\mathbb{C}^{3}\right)_{\mathrm{c}(\omega)} .
$$

Take $\phi$ and $\psi$ in $\operatorname{Bir}\left(\mathbb{C}^{3}\right)_{\mathrm{c}(\omega)}$ such that $\alpha(\phi)=\alpha(\psi)$, then $\psi_{1}$ and $\psi_{2}$ are first integrals of $Z_{\phi}$ and by Proposition 3.1.4

$$
\psi_{1}=\varphi_{1}\left(\phi_{1}, \phi_{2}\right), \quad \psi_{2}=\varphi_{2}\left(\phi_{1}, \phi_{2}\right)
$$

where $\varphi=\left(\varphi_{1}, \varphi_{2}\right)$ is birational. Hence

$$
\psi \phi^{-1}=\left(\psi_{0} \circ \phi^{-1}, \varphi_{1}\left(z_{1}, z_{2}\right), \varphi_{2}\left(z_{1}, z_{2}\right)\right)
$$

belongs to $\mathscr{K}$; in other words $\phi$ and $\psi$ are in the same $\mathscr{K}$-orbit.

Assume now that $\psi=\kappa \phi$ where $\kappa$ denotes an element of $\mathscr{K}$. Then the foliations defined by $Z_{\phi}$ and $Z_{\psi}$ coincide because they have the same set of first integrals. As a consequence $\alpha(\phi)=\alpha(\psi)$.

Hence one can state:

THEOREM 3.1.5. The map $\alpha$ is a complete invariant of the left translation action of $\mathscr{K}$ on $\operatorname{Bir}\left(\mathbb{C}^{3}\right)_{\mathrm{c}(\omega)}$, that is for any $\phi$ and $\psi$ in $\operatorname{Bir}\left(\mathbb{C}^{3}\right)_{\mathrm{c}(\omega)}$ one has $\alpha(\phi)=\alpha(\psi)$ if and only if $\psi \phi^{-1}$ belongs to $\mathscr{K}$.

Question 1. Is the map $\alpha$ surjective?

Let us consider the following differential equation

$$
y^{\prime \prime}=F\left(x, y, y^{\prime}\right)
$$

where $F$ denotes a rational function. Set $y^{\prime}=u$, then

$$
(3.1) \Leftrightarrow\left\{\begin{array}{l}
\frac{d u}{d t}=F(x, y, u), \\
\frac{d y}{d t}=u, \\
\frac{d x}{d t}=1 .
\end{array}\right.
$$

So one can associate to (3.1) the following vector field

$$
Z=F \frac{\partial}{\partial u}+u \frac{\partial}{\partial y}+\frac{\partial}{\partial x} .
$$


We say that (3.1) is rationally integrable if the vector field $Z$ has two first integrals $r_{1}$ and $r_{2}$ rationally independent: $\mathrm{d} r_{1} \wedge \mathrm{d} r_{2} \not \equiv 0$.

For generic $\gamma$ and $\beta$ in $\mathbb{C}$ the differential equation $y^{\prime \prime}+\gamma y^{\prime}+\beta y=0$ is not rationally integrable; as a consequence $-\gamma z_{0}-\beta z_{2}$ is not in the image of $\alpha$. The first Painlevé equation gives examples of polynomial of degree 2 that does not belong to $\operatorname{im} \alpha$ :

TheOREM 3.1.6 ([12]). The equation $\mathcal{P}_{1}$

$$
y^{\prime \prime}=6 y^{2}+x
$$

is not rationally integrable.

If we come back with our notations it means that $6 z_{2}^{2}-z_{1}$ is not in the image of $\alpha$.

REMARK 3.1.7. Indeed all generic Painlevé equations give rise to rational functions that do not belong to $\operatorname{im} \alpha$.

Nevertheless one can easily obtain examples of elements in the image of $\alpha$ :

ExAmples 3.1.8. - If $\phi=\left(z_{0} / \beta, z_{0}+\beta z_{1}, z_{2}-z_{0}^{2} / 2 \beta\right)$ with $\beta \in \mathbb{C}^{*}$, then $\alpha(\phi)=\beta$.

- If

$$
\phi=\left(z_{0}, z_{1}+P\left(z_{0}\right), z_{2}+Q\left(z_{0}\right)\right)
$$

with $P, Q$ in $\mathbb{C}\left[z_{0}\right]$ such that $Q^{\prime}\left(z_{0}\right)=-z_{0} P^{\prime}\left(z_{0}\right)$, then $\alpha(\phi)=1 / P^{\prime}\left(z_{0}\right)$.

- If

$$
\phi=\left(-z_{1}, z_{0}+P\left(z_{1}\right), z_{2}+z_{0} z_{1}+Q\left(z_{1}\right)\right)
$$

with $P, Q$ in $\mathbb{C}\left[z_{1}\right]$ such that $Q^{\prime}\left(z_{1}\right)=z_{1} P^{\prime}\left(z_{1}\right)$ then $\alpha(\phi)=P^{\prime}\left(z_{1}\right)$.

Consider the left translation action of $\operatorname{Bir}\left(\mathbb{C}^{3}\right)_{\omega}$ on $\operatorname{Bir}\left(\mathbb{C}^{3}\right)_{c(\omega)}$ defined by

$$
(\psi, \phi) \in \operatorname{Bir}\left(\mathbb{C}^{3}\right)_{\omega} \times \operatorname{Bir}\left(\mathbb{C}^{3}\right)_{\mathrm{c}(\omega)} \longrightarrow \psi \phi \in \operatorname{Bir}\left(\mathbb{C}^{3}\right)_{\mathrm{c}(\omega)}
$$

TheORem 3.1.9. The map $V$ is a complete invariant of the left translation action of $\operatorname{Bir}\left(\mathbb{C}^{3}\right)_{\omega}$ on $\operatorname{Bir}\left(\mathbb{C}^{3}\right)_{\mathrm{c}(\omega)}$ : for any $\phi, \psi$ in $\operatorname{Bir}\left(\mathbb{C}^{3}\right)_{\mathrm{c}(\omega)}$ one has $V(\phi)=V(\psi)$ if and only if $\psi \phi^{-1}$ belongs to $\operatorname{Bir}\left(\mathbb{C}^{3}\right)_{\omega}$.

Proof. Let $\phi$ be a contact birational map of $\mathbb{P}^{3}$. Obviously $(f \phi)^{*} \omega=V(\phi) \omega$ for any $f \in \operatorname{Bir}\left(\mathbb{C}^{3}\right)_{\omega}$.

Let us now consider two contact birational maps $\phi$ and $\psi$ of $\mathbb{P}^{3}$ such that $V=$ $V(\phi)=V(\psi)$. On the one hand

$$
\left(\phi^{-1}\right)^{*} \psi^{*} \omega=\left(\phi^{-1}\right)^{*} V(\phi) \omega=V \circ \phi^{-1}\left(\phi^{-1}\right)^{*} \omega
$$

and on the other hand composing $\phi^{*} \omega=V \omega$ by $\left(\phi^{-1}\right)^{*}$ one gets 


$$
\phi^{*} \omega=V \omega \Rightarrow\left(\phi^{-1}\right)^{*}\left(\phi^{*} \omega\right)=\left(\phi^{-1}\right)^{*}(V \omega) \Rightarrow \omega=V \circ \phi^{-1}\left(\phi^{-1}\right)^{*} \omega .
$$

As a consequence $\left(\phi^{-1}\right)^{*} \psi^{*} \omega=\omega$, that is $\psi \phi^{-1}$ belongs to $\operatorname{Bir}\left(\mathbb{C}^{3}\right)_{\omega}$.

Proposition 3.1.10. If $\phi$ and $\psi$ are two contact birational maps of $\mathbb{P}^{3}$ such that $\alpha(\phi)=\alpha(\psi)$ and $V(\phi)=V(\psi)$, then $\psi \phi^{-1}$ belongs to

$$
\left\{\left(\frac{z_{0}-b^{\prime}\left(z_{1}\right)}{\nu^{\prime}\left(z_{1}\right)}, \nu\left(z_{1}\right), z_{2}+b\left(z_{1}\right)\right) \mid b \in \mathbb{C}\left(z_{1}\right), \nu \in P G L(2 ; \mathbb{C})\right\}=\mathscr{K} \cap \operatorname{Bir}\left(\mathbb{C}^{3}\right)_{\omega} .
$$

Proof. Since both $\alpha(\phi)=\alpha(\psi)$ and $V(\phi)=V(\psi)$ the map $\psi \phi^{-1}$ is an element of $\operatorname{Bir}\left(\mathbb{C}^{3}\right)_{\omega} \cap \mathscr{K}$. One gets the result from the descriptions of the Klein family and of $\operatorname{Bir}\left(\mathbb{C}^{3}\right)_{\omega}$ (Proposition 2.2.1).

Let us now give some examples of $V(\phi)$.

EXAmPles 3.1.11. • If $\phi$ belongs to $\mathscr{K}$, then

$$
V(\phi)=\frac{\partial \phi_{1} / \partial z_{1} \cdot \partial \phi_{2} / \partial z_{2}-\partial \phi_{1} / \partial z_{2} \cdot \partial \phi_{2} / \partial z_{1}}{\partial \phi_{1} / \partial z_{1}-z_{0} \partial \phi_{1} / \partial z_{2}}
$$

- If

$$
\phi=\left(\frac{1}{n z_{0}^{n-1} z_{2}+(n+1) z_{0}^{n}\left(z_{1}+1\right)}, z_{0}^{n}\left(z_{0}+z_{2}+z_{0} z_{1}\right),-z_{0}\right)
$$

with $n \in \mathbb{Z}$, then $V(\phi)=z_{0} /\left((n+1) z_{0} z_{1}+n z_{2}+(n+1) z_{0}\right)$.

- If

$$
\phi=\left(\frac{\left(z_{1}-z_{0}\right)^{2}}{2 z_{0} z_{1}+2 z_{2}-z_{0}^{2}}, \frac{2 z_{2}+z_{0}^{2}}{z_{1}-z_{0}}, z_{1}-z_{0}\right)
$$

then $V(\phi)=2\left(z_{0}-z_{1}\right) /\left(z_{0}^{2}-2 z_{0} z_{1}-2 z_{2}\right)$.

REMARK 3.1.12. If $\phi$ belongs to $\operatorname{Bir}\left(\mathbb{C}^{3}\right)_{\mathrm{c}(\omega)}$, then $\phi^{*} \omega=V(\phi) \omega$ and $\phi^{*}(\omega \wedge \mathrm{d} \omega)=$ $V(\phi)^{2} \omega \wedge \mathrm{d} \omega$ and $\operatorname{det} \mathrm{jac} \phi$ is a square. This gives some constraint on $V(\phi)$.

As previously we can ask: is $V$ surjective? The answer is no. Indeed let us assume that there exists $\phi \in \operatorname{Bir}\left(\mathbb{C}^{3}\right)_{\mathrm{c}(\omega)}$ such that $V(\phi)=z_{2}$. Then $\phi_{0} \mathrm{~d} \phi_{0}+\mathrm{d} \phi_{2}=z_{0} z_{2} \mathrm{~d} z_{1}+$ $\mathrm{d}\left(z_{2}^{2} / 2\right)$ and $\mathrm{d} \phi_{0} \wedge \mathrm{d} \phi_{1}=\mathrm{d}\left(z_{0} z_{2}\right) \wedge \mathrm{d} z_{1}$. Since the fibers of $\left(z_{0} z_{2}, z_{1}\right)$ are connected one can write $\phi_{0}$ as $\varphi_{0}\left(z_{0} z_{2}, z_{1}\right)$ and $\phi_{1}$ as $\varphi_{1}\left(z_{0} z_{2}, z_{1}\right)$. Then $\phi^{*} \omega=V(\phi) \omega$ implies that $\phi_{2}-z_{2}^{2} / 2=\varphi_{2}\left(z_{0} z_{2}, z_{1}\right)$. In other words

$$
\phi=\left(\varphi_{0}\left(z_{0} z_{2}, z_{1}\right), \varphi_{1}\left(z_{0} z_{2}, z_{1}\right), \varphi_{2}\left(z_{0} z_{2}, z_{1}\right)+\frac{z_{2}^{2}}{2}\right) .
$$

But $\phi \circ\left(z_{0} / z_{2}, z_{1}, z_{2}\right)$ is clearly not birational so does $\phi$ : contradiction. 


\subsection{Invariant forms and vector fields.}

The next statement deals with flows in $\operatorname{Bir}\left(\mathbb{C}^{3}\right)_{\omega}$ (see $[\mathbf{1 3}]$ for a definition).

Proposition 3.2.1. Let $\phi_{t}$ be a flow in $\operatorname{Bir}\left(\mathbb{C}^{3}\right)_{\omega}$. Then $\phi_{t}$ has a first integral depending only on $\left(z_{0}, z_{1}\right)$ and with rational fibers.

In other words

$$
\phi_{t}=\left(\varphi_{t}\left(z_{0}, z_{1}\right), z_{2}+b_{t}\left(z_{0}, z_{1}\right)\right)
$$

where $\varphi_{t}$ belongs, up to conjugacy, to $\mathcal{J}$ and $b_{t}$ to $\mathbb{C}\left(z_{0}, z_{1}\right)$.

Proof. Let $\chi$ be the infinitesimal generator of $\phi_{t}$, i.e.

$$
\chi=\left.\frac{\partial \phi_{t}}{\partial t}\right|_{t=0} .
$$

By derivating $\phi_{t}^{*} \omega=\omega$ with respect to $t$ one gets that the Lie derivative $L_{\chi} \omega$ is zero. Set $\chi=\sum_{i=0}^{2} \chi_{i} \partial / \partial z_{i}$, hence

$$
L_{\chi} \omega=\iota_{\chi} \mathrm{d} \omega+\mathrm{d} \iota_{\chi} \omega=\chi_{0} \mathrm{~d} z_{1}+z_{0} \mathrm{~d} \chi_{1}+\mathrm{d} \chi_{2}
$$

and so

$$
L_{\chi} \omega=\left(z_{0} \frac{\partial \chi_{1}}{\partial z_{0}}+\frac{\partial \chi_{2}}{\partial z_{0}}\right) \mathrm{d} z_{0}+\left(\chi_{0}+z_{0} \frac{\partial \chi_{1}}{\partial z_{1}}+\frac{\partial \chi_{2}}{\partial z_{1}}\right) \mathrm{d} z_{1}+\left(z_{0} \frac{\partial \chi_{1}}{\partial z_{2}}+\frac{\partial \chi_{2}}{\partial z_{2}}\right) \mathrm{d} z_{2} .
$$

In particular $z_{0} \chi_{1}+\chi_{2}=\gamma\left(z_{0}, z_{1}\right)$, then $\chi_{0}+\left(\partial / \partial z_{1}\right)\left(z_{0} \chi_{1}+\chi_{2}\right)=0$ so $\chi_{0}=-\partial \gamma / \partial z_{1}$ and finally $\chi_{1}=\partial \gamma / \partial z_{0}$.

If $\gamma$ is constant, then $\chi=\partial / \partial z_{2}$, that is $\phi_{t}=\left(z_{0}, z_{1}, z_{2}+\beta t\right)$ with $\beta \in \mathbb{C}$.

Let us now assume that $\gamma$ is non-constant; one has

$$
\chi=-\frac{\partial \gamma}{\partial z_{1}} \frac{\partial}{\partial z_{0}}+\frac{\partial \gamma}{\partial z_{0}} \frac{\partial}{\partial z_{1}}+\left(\gamma\left(z_{0}, z_{1}\right)-z_{0} \frac{\partial \gamma}{\partial z_{0}}\right) \frac{\partial}{\partial z_{2}}
$$

and $\gamma$ is a first integral of $\chi$. For all $t$

$$
\phi_{t}=\left(\phi_{0, t}\left(z_{0}, z_{1}\right), \phi_{1, t}\left(z_{0}, z_{1}\right), z_{2}+b_{t}\left(z_{0}, z_{1}\right)\right)
$$

and the function $\gamma$ is invariant by $\phi_{t}$ and as a consequence by the flow $\varphi_{t}$. The fibers of $\gamma$ in $\mathbb{C}^{2}$ (up to compactification/normalization) are rational or elliptic since they own a flow. As $\left\langle\varphi_{t}\right\rangle$ is uncountable they have to be rational $([\mathbf{9}])$ and up to conjugacy $\varphi_{t}$ belongs to $\mathcal{J}$.

The following examples contain many flows.

ExAmple 3.2.2. The elements of $\operatorname{Aut}\left(\mathbb{P}^{3}\right)_{\mathrm{c}(\omega)}$ can be written

$$
\left(\varepsilon z_{0}+\lambda, \beta z_{1}+\gamma,-\beta \lambda z_{1}+\varepsilon \beta z_{2}+\delta\right)
$$

with $\varepsilon, \beta$ in $\mathbb{C}^{*}$ and $\lambda, \gamma, \delta$ in $\mathbb{C}$. The group $\operatorname{Aut}\left(\mathbb{P}^{3}\right)_{\mathrm{c}(\omega)}$ acts transitively on $\mathbb{C}^{3}=\left\{z_{3}=1\right\}$. 
Examples 3.2.3. a) For any $\varepsilon, \beta, \gamma$ and $\delta$ in $\mathbb{C}$ such that $\varepsilon \delta-\beta \gamma \neq 0$, the map

$$
\left(\frac{\left(\gamma z_{1}+\delta\right)^{2}}{\varepsilon \delta-\beta \gamma} z_{0}, \frac{\varepsilon z_{1}+\beta}{\gamma z_{1}+\delta}, z_{2}\right)
$$

belongs to $\operatorname{Bir}\left(\mathbb{C}^{3}\right)_{\omega}$. These maps form a group contained in $\operatorname{im} \mathcal{K}$ and isomorphic to $P G L(2 ; \mathbb{C})$.

b) The birational maps given by

- $\left(z_{0}, z_{1}+\varphi\left(z_{0}\right), z_{2}+\psi\left(z_{0}\right)\right)$ with $z_{0} \varphi^{\prime}\left(z_{0}\right)+\psi^{\prime}\left(z_{0}\right)=0$,

- $\left(z_{0}-\psi^{\prime}\left(z_{1}\right), z_{1}, z_{2}+\psi\left(z_{1}\right)\right)$

belong to $\operatorname{Bir}\left(\mathbb{C}^{3}\right)_{\omega}$. Any of these families forms an abelian group.

The fact that an element of $\operatorname{Bir}\left(\mathbb{C}^{3}\right)_{c(\omega)}$ preserves a vector field and the fact that it preserves a contact form are related:

Proposition 3.2.4. Let $\phi$ be a contact birational map of $\mathbb{P}^{3}$. There exist a contact form $\Theta$ colinear to $\omega$ such that $\phi^{*} \Theta=\Theta$ if and only if $V(\phi)$ can be written $U / U \circ \phi$ for some rational function $U$. In that case $\phi$ preserves the Reeb flow associated to $\Theta$, so a foliation by curves.

Proof. Assume that such a $\Theta$ exists. On the one hand $\phi^{*} \omega=V(\phi) \omega$ and on the other hand $\Theta=U \omega$. Hence

$$
\phi^{*} \Theta=U \circ \phi \cdot \phi^{*} \omega=U \circ \phi \cdot V(\phi) \omega=\frac{U \circ \phi}{U} \cdot V(\phi) \Theta
$$

and so if such $U$ exists, one has $V(\phi)=U / U \circ \phi$.

Reciprocally if $\phi \in \operatorname{Bir}\left(\mathbb{C}^{3}\right)_{c(\omega)} \backslash \operatorname{Bir}\left(\mathbb{C}^{3}\right)_{\omega}$ satisfies $\phi^{*} \omega=(U / U \circ \phi) \omega$ for some rational function $U$, then $\phi^{*} \Theta=\Theta$ where $\Theta=U \omega$.

EXAMPLES 3.2.5. - First consider the Legendre involution $\mathcal{L}=\left(z_{1}, z_{0},-z_{2}-\right.$ $\left.z_{0} z_{1}\right)$. As we have seen $V(\mathcal{L})=-1$. One can check that $U=z_{2}+\left(z_{0} z_{1} / 2\right)$ suits.

- For an element $\phi$ in $\operatorname{Aut}\left(\mathbb{P}^{3}\right)_{\mathrm{c}(\omega)}$

$$
\phi=\left(\varepsilon z_{0}+\lambda, \beta z_{1}+\gamma,-\beta \lambda z_{1}+\varepsilon \beta z_{2}+\delta\right)
$$

with $\varepsilon, \beta$ in $\mathbb{C}^{*}$ and $\lambda, \gamma, \delta$ in $\mathbb{C}$ (Example 3.2.2) we have $V(\phi)=\varepsilon \beta$. If

$$
U=\frac{\varepsilon \beta}{\varepsilon \beta z_{0} z_{1}+\varepsilon \gamma z_{0}+\beta \lambda z_{1}+\lambda \gamma}
$$

then $V(\phi)=U / U \circ \phi$.

Proposition 3.2.6. Let $\phi$ be an element of $\operatorname{Bir}\left(\mathbb{C}^{3}\right)_{\mathrm{c}(\omega)} \backslash \operatorname{Bir}\left(\mathbb{C}^{3}\right)_{\omega}$. Assume that $\phi$ preserves a vector field $\chi$ non-tangent to $\omega$. Then $\phi$ preserves a contact form $\omega^{\prime}$ colinear to $\omega$. 
REMARK 3.2.7. Under these assumptions $\phi$ preserves the vector field $\chi$ and the Reeb vector field $Z$ associated to $\omega^{\prime}$. With the previous notations if $f=z_{0} \chi_{1}+\chi_{2}$ and $g=z_{0} Z_{1}+Z_{2}$ one has $V(\phi)=f \circ \phi / f=g \circ \phi / g$. In particular if $H=f / g$ is non-constant, then $H$ is non-constant and invariant: $H \circ \phi=H$.

Proof of Proposition 3.2.6. Write $\chi$ as $\chi_{0} \partial / \partial z_{0}+\chi_{1} \partial / \partial z_{1}+\chi_{2} \partial / \partial z_{2}$ and $\phi$ as $\left(\phi_{0}, \phi_{1}, \phi_{2}\right)$. Then $\phi_{*} \chi=\chi$ if and only if $\mathrm{d} \phi_{i}(\chi)=\chi_{i} \circ \phi$ for $i=0,1$ and 2 . Therefore $\phi^{*} \omega(\chi)=V(\phi) \omega(\chi)$ can be rewritten

$$
\phi_{0} \mathrm{~d} \phi_{1}(\chi)+\mathrm{d} \phi_{2}(\chi)=\phi_{0} \chi_{1} \circ \phi+\chi_{2} \circ \phi=V(\phi)\left(z_{0} \chi_{1}+\chi_{2}\right) .
$$

The vector field $\chi$ is not tangent to $\omega$, i.e. $\omega(\chi) \not \equiv 0$ or in other words $z_{0} \chi_{1}+\chi_{2} \not \equiv 0$ and so

$$
V(\phi)=\frac{\left(z_{0} \chi_{1}+\chi_{2}\right) \circ \phi}{z_{0} \chi_{1}+\chi_{2}} .
$$

As a consequence $\phi$ preserves a contact form $\omega^{\prime}$ colinear to $\omega$ (Proposition 3.2.4).

REMARK 3.2.8. Let $\phi \in \operatorname{Bir}\left(\mathbb{C}^{3}\right)_{\mathrm{c}(\omega)} \backslash \operatorname{Bir}\left(\mathbb{C}^{3}\right)_{\omega}$. Assume that there exists a vector field $\chi$ such that $\phi_{*} \chi=W \chi$. If $W$ can be written $U \circ \phi / U$, then $\phi$ preserves the vector field $Y=U \chi$. According to Proposition 3.2.6 the map $\phi$ belongs to $\operatorname{Bir}\left(\mathbb{C}^{3}\right)_{\omega^{\prime}}$ where $\omega^{\prime}$ denotes a contact form colinear to $\omega$.

\subsection{Regular birational maps.}

Let $\boldsymbol{e}_{i}$ be the point of $\mathbb{P}_{\mathbb{C}}^{3}$ whose all components are zero except the $i$-th.

Let us denote by $\mathcal{H}_{\infty}$ the hyperplane $z_{3}=0$. As $\mathcal{H}_{\infty}$ is the unique invariant surface of $\mathrm{c}(\omega)$ one has the following statement:

Proposition 3.3.1. The hyperplane $\mathcal{H}_{\infty}$ is either preserved, or blown down by any element of $\operatorname{Bir}\left(\mathbb{C}^{3}\right)_{\mathrm{c}(\omega)}$.

EXAMPLE 3.3.2. Let $\varphi$ be a birational map of the complex projective plane; $\mathcal{K}(\varphi)$ is polynomial if and only if $\varphi=\left(\beta z_{1}+\gamma, \delta z_{2}+P\left(z_{1}\right)\right)$ with $P \in \mathbb{C}\left[z_{1}\right]$; remark that such a $\varphi$ is a Jonquières polynomial automorphism. In that case

$$
\mathcal{K}(\varphi)=\left(\frac{1}{\beta}\left(\delta z_{0}-\frac{\partial P\left(z_{1}\right)}{\partial z_{1}}\right), \beta z_{1}+\gamma, \delta z_{2}+P\left(z_{1}\right)\right) .
$$

Note that $\operatorname{deg} P=1$ if and only if $\mathcal{K}(\varphi)$ is an automorphism of $\mathbb{P}^{3}$. If $\operatorname{deg} P>1$, then Ind $\mathcal{K}(\varphi)=\left\{z_{1}=z_{3}=0\right\}$ and $\mathcal{H}_{\infty}$ is blown down onto $\boldsymbol{e}_{3}$.

Proposition 3.3.1 naturally implies the following definition. We say that $\phi \in$ $\operatorname{Bir}\left(\mathbb{C}^{3}\right)_{c(\omega)}$ is regular at infinity if $\mathcal{H}_{\infty}$ is preserved by $\phi$ and if $\phi_{\mid \mathcal{H}_{\infty}}$ is birational. We denote by $\operatorname{Bir}\left(\mathbb{C}^{3}\right)_{\mathrm{c}(\omega)}^{\mathrm{reg}}\left(\right.$ resp. $\left.\operatorname{Bir}\left(\mathbb{C}^{3}\right)_{\omega}^{\mathrm{reg}}\right)$ the set of regular maps at infinity that belong to $\operatorname{Bir}\left(\mathbb{C}^{3}\right)_{c(\omega)}\left(\right.$ resp. $\left.\operatorname{Bir}\left(\mathbb{C}^{3}\right)_{\omega}\right)$.

ExAmple 3.3.3. Of course the elements of $\operatorname{Aut}\left(\mathbb{P}^{3}\right)_{c(\omega)}$ (Example 3.2.2) are regular at infinity. 
The contact structure is also given in homogeneous coordinates by the 1-form

$$
\bar{\omega}=z_{0} z_{3} \mathrm{~d} z_{1}+z_{3}^{2} \mathrm{~d} z_{2}-\left(z_{0} z_{1}+z_{2} z_{3}\right) \mathrm{d} z_{3} .
$$

Let $\phi$ be an element of $\operatorname{Bir}\left(\mathbb{C}^{3}\right)_{\mathrm{c}(\omega)}^{\mathrm{reg}}$; denote by $\bar{\phi}$ its homogeneization. Since $\phi^{*} \omega=V(\phi) \omega$ one has $\bar{\phi}^{*} \bar{\omega}=\overline{V(\phi)} \bar{\omega}$ where $\overline{V(\phi)}$ is a homogeneous polynomial. With these notations one can state:

LEMMA 3.3.4. Let $\phi$ be a contact birational map of $\mathbb{P}^{3}$. Assume that $\phi$ either preserves $\mathcal{H}_{\infty}$, or blows down $\mathcal{H}_{\infty}$ onto a subset contained in $\mathcal{H}_{\infty}$.

The map $\phi$ is regular if and only if $\overline{V(\phi)}$ does not vanish identically on $\mathcal{H}_{\infty}$.

Proof. Let us work in the affine chart $z_{2}=1$. On the one hand

$$
\bar{\omega} \wedge \mathrm{d} \bar{\omega}=-z_{3}^{2} \mathrm{~d} z_{0} \wedge \mathrm{d} z_{1} \wedge \mathrm{d} z_{3}
$$

and on the other hand

$$
\phi^{*}(\bar{\omega} \wedge \mathrm{d} \bar{\omega})=\overline{V(\phi)}^{2} \bar{\omega} \wedge \mathrm{d} \bar{\omega}
$$

Hence

$$
\bar{\phi}_{3}^{2} \operatorname{det} \mathrm{jac} \bar{\phi}=\overline{V(\phi)}^{2} z_{3}^{2}
$$

where $\bar{\phi}_{3}$ is the third component of $\bar{\phi}$ expressed in the affine chart $z_{2}=1$.

Suppose that $\phi$ is regular. Let $p$ be a generic point of $\mathcal{H}_{\infty}$. As $\phi$ is regular, $\bar{\phi}_{\mid \mathcal{H}_{\infty}}$ is a local diffeomorphism at $p$. Since $\bar{\phi}$ is birational and $p$ is generic, $\bar{\phi}_{, p}$ is a local diffeomorphism. As a consequence $\operatorname{det} \mathrm{jac} \bar{\phi}$ is an unit at $p$; moreover the invariance of $\mathcal{H}_{\infty}$ by $\bar{\phi}$ implies that $\bar{\phi}_{3}=z_{3} u$ where $u$ is a unit. Therefore $\overline{V(\phi)}$ does not vanish at $p$.

Conversely assume that $\overline{V(\phi)}$ does not vanish identically on $\mathcal{H}_{\infty}$. As $\phi$ either preserves $\mathcal{H}_{\infty}$, or contracts $\mathcal{H}_{\infty}$ onto a subset in $\mathcal{H}_{\infty}$, one can write $\bar{\phi}_{3}$ as $z_{3} P$. As a result

$$
\Leftrightarrow \quad P^{2} \operatorname{det} \operatorname{jac} \bar{\phi}=\overline{V(\phi)}^{2}
$$

Since $\overline{V(\phi)}$ does not vanish the map $\phi$ is then regular at infinity.

Corollary 3.3.5. One has $\operatorname{Bir}\left(\mathbb{C}^{3}\right)_{\omega}^{\mathrm{reg}}=\operatorname{Aut}\left(\mathbb{P}^{3}\right)_{\omega}$.

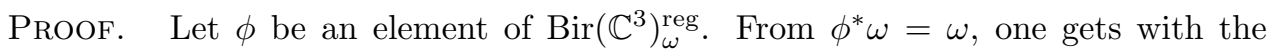
previous notations $\bar{\phi}^{*} \bar{\omega}=z_{3}^{n} \bar{\omega}$ for some integer $n$. Lemma 3.3.4 implies that $n=0$, that is $\bar{\phi}^{*} \bar{\omega}=\bar{\omega}$; then looking at the degree of the members of this equality one gets $\operatorname{deg} \phi=1$.

EXAmPle 3.3.6. The group $\operatorname{Bir}\left(\mathbb{C}^{3}\right)_{\mathrm{c}(\omega)}^{\mathrm{reg}}$ contains blow-ups in restriction to $\mathcal{H}_{\infty}$. Indeed let us look at $\omega$ in the affine chart $z_{2}=1$ and consider the birational map $\phi$ given in $z_{2}=1$ by

$$
\phi=\left(z_{0}, z_{0} z_{1}-z_{3}, z_{0} z_{3}\right)
$$


Since $\left(\phi^{n}\right)^{*} \omega=z_{0}^{-n} \omega, \phi^{n} \in \operatorname{Bir}\left(\mathbb{C}^{3}\right)_{\mathrm{c}(\omega)}^{\mathrm{reg}} \backslash \operatorname{Bir}\left(\mathbb{C}^{3}\right)_{\omega}$ for any $n \neq 0$; in restriction to $\mathcal{H}_{\infty}$ the map $\phi^{n}$ coincides with $\left(z_{0}, z_{1} z_{0}^{n}\right)$.

Let us note that Ind $\phi^{n}=\left\{\boldsymbol{e}_{1}\right\} \cup\left(z_{0}=z_{2}=0\right)$, that $z_{0}=0$ is contracted by $\phi$ onto $\left(z_{0}=z_{2}=0\right)$ and $z_{2}=0$ onto $\left(z_{0}=z_{3}=0\right)$. Besides Ind $\phi^{-n}=\left\{z_{0}=z_{2}=0\right\} \cup\left\{z_{0}=\right.$ $\left.z_{3}=0\right\},\left(z_{0}=0\right)$ is blown down by $\phi^{-1}$ onto $\boldsymbol{e}_{2}$ and $\left(z_{2}=0\right)$ onto $\boldsymbol{e}_{1}$.

REMARK 3.3.7. The group generated by Examples 3.3.3 and 3.3.6 is in restriction to $\mathcal{H}_{\infty}$ and in the affine chart $z_{2}=1$

$$
\left\langle\left(\frac{\gamma z_{0}}{\beta z_{1}+\lambda}, \frac{\lambda z_{1}}{\gamma\left(\beta z_{1}+\lambda\right)}\right),\left(z_{0}, z_{0} z_{1}\right) \mid \gamma, \beta \in \mathbb{C}^{*}, \lambda \in \mathbb{C}\right\rangle
$$

it is of course a subgroup of $\operatorname{Bir}\left(\mathbb{C}^{3}\right)_{\mathrm{c}(\omega)}^{\mathrm{reg}}$.

Question 2. Does this group coincide with $\operatorname{Bir}\left(\mathbb{C}^{3}\right)_{\mathrm{c}(\omega)}^{\mathrm{reg}}$ ?

ExAMPLES 3.3.8. a) If $\phi$ is either a monomial map (i.e. a map of the form $\left(z_{1}^{p} z_{2}^{q}, z_{1}^{r} z_{2}^{s}\right)$ with $\left[\begin{array}{cc}p & q \\ r & s\end{array}\right]$ in $\left.G L(2 ; \mathbb{Z})\right)$, or a non-linear polynomial automorphism, or a Jonquières map, then $\mathcal{K}(\phi)$ is not regular at infinity.

b) The map of order 5 given by $\left(-\left(z_{2}+1+z_{0} z_{1}\right) / z_{0} z_{1}^{2}, z_{2},\left(z_{2}+1\right) / z_{1}\right)$, the map $\left(z_{0} /\left(z_{2}+1\right)^{2}, z_{1}, z_{2} /\left(z_{2}+1\right)\right)$ and Examples 3.2.3 a) are non-regular at infinity.

c) Any map of the form

$$
\left(\frac{1}{z_{0}}-f^{\prime}\left(z_{2}\right), z_{2}, z_{1}+f\left(z_{2}\right)\right)
$$

is in $\operatorname{Bir}\left(\mathbb{C}^{3}\right)_{\mathrm{c}(\omega)} \backslash \operatorname{Bir}\left(\mathbb{C}^{3}\right)_{\omega}$ and is not regular at infinity.

d) Elements of the Legendre family are not regular at infinity.

\subsection{Exact birational maps.}

Recall that an element $\phi$ of $\operatorname{Bir}\left(\mathbb{C}^{2}\right)_{\eta}$ is exact if it can be lifted via $\varsigma$ to $\operatorname{Bir}\left(\mathbb{C}^{3}\right)_{\omega}$, or equivalently if it belongs to im $\varsigma$. The following statement allows to determine such maps.

TheOREM 3.4.1. A map $\left(\phi_{0}\left(z_{0}, z_{1}\right), \phi_{1}\left(z_{0}, z_{1}\right)\right) \in \operatorname{Bir}\left(\mathbb{C}^{2}\right)_{\eta}$ is exact if and only if the closed form $\phi_{0} \mathrm{~d} \phi_{1}-z_{0} \mathrm{~d} z_{1}$ has trivial residues. In that case $\phi_{0} \mathrm{~d} \phi_{1}-z_{0} \mathrm{~d} z_{1}=-\mathrm{d} b$ with $b \in \mathbb{C}\left(z_{0}, z_{1}\right)$ and

$$
\phi=\left(\phi_{0}\left(z_{0}, z_{1}\right), \phi_{1}\left(z_{0}, z_{1}\right), z_{2}+b\left(z_{0}, z_{1}\right)\right)
$$

belongs to $\operatorname{Bir}\left(\mathbb{C}^{3}\right)_{\omega}$.

Proof. Remark that $\phi=\left(\phi_{0}\left(z_{0}, z_{1}\right), \phi_{1}\left(z_{0}, z_{1}\right), z_{2}+b\left(z_{0}, z_{1}\right)\right)$ belongs to $\operatorname{Bir}\left(\mathbb{C}^{3}\right)_{\omega}$ if and only if

$$
\phi_{0} \mathrm{~d} \phi_{1}-z_{0} \mathrm{~d} z_{1}=-\mathrm{d} b
$$


in other words $\phi_{0} \mathrm{~d} \phi_{1}-z_{0} \mathrm{~d} z_{1}$ is not only a closed rational 1-form but also an exact one. Recall that a closed rational 1-form $\Theta$ can be written $([\mathbf{1 4}])$

$$
\Theta=\sum_{i} \lambda_{i} \frac{\mathrm{d} f_{i}}{f_{i}}+\mathrm{d} g
$$

where the $\lambda_{i}$ are complex numbers and the $f_{i}$ 's and $g$ are rational. The 1 -form $\Theta$ is exact (i.e. the differential of a rational function) if $\lambda_{i}=0$ for all $i$, that is if the residues of $\Theta$ are trivial.

EXAmple 3.4.2. The set

$$
\left\{\left(A\left(z_{0}\right), \frac{z_{1}}{A^{\prime}\left(z_{0}\right)}\right) \mid A \in P G L(2 ; \mathbb{C})\right\}
$$

is a subgroup of exact maps isomorphic to $P G L(2 ; \mathbb{C})$; it is a direct consequence of Theorem 3.4.1.

An other direct consequence of Theorem 3.4.1 is the following statement:

Corollary 3.4.3. The maps $\phi=\left(\phi_{0}, \phi_{1}\right)$ of $\operatorname{Bir}\left(\mathbb{C}^{2}\right)_{\eta}$ such that $\phi_{0} \mathrm{~d} \phi_{1}-z_{0} \mathrm{~d} z_{1}$ has trivial residues form a group.

Let us deal with exact birational involutions.

Bertini gives a classification of birational involutions $([\mathbf{6}])$ : a non-trivial birational involution is conjugate to either a Jonquières involution of degree $\geq 2$, or a Bertini involution, or a Geiser involution. More recently Bayle and Beauville precise it ([5]); the map which associates to a birational involution of $\mathbb{P}^{2}$ its normalized fixed curve establishes a one-to-one correspondence between:

- conjugacy classes of Jonquières involutions of degree $d$ and isomorphism classes of hyperelliptic curves of genus $d-2(d \geq 3)$;

- conjugacy classes of Geiser involutions and isomorphism classes of non-hyperelliptic curves of genus 3 ;

- conjugacy classes of Bertini involutions and isomorphism classes of nonhyperelliptic curves of genus 4 whose canonical model lies on a singular quadric.

Besides the Jonquières involutions of degree 2 form one conjugacy class.

Proposition 3.4.4. Let $\mathcal{I} \in \operatorname{Bir}\left(\mathbb{P}^{2}\right)$ be a birational involution. If $\mathcal{I}$ is conjugate to either a Geiser involution, or a Bertini involution, or a Jonquières involution of degree $\geq 3$, then $\mathcal{I}$ does not belong to $\operatorname{Bir}\left(\mathbb{C}^{2}\right)_{\eta}$.

Hence the only involutions in $\operatorname{Bir}\left(\mathbb{C}^{2}\right)_{\eta}$ are birationally conjugate to $\left(-z_{0},-z_{1}\right)$. Some of them can not be lifted.

Proof. Let us consider such an involution, then the set of fixed points contains a curve $\Gamma$ of genus $>0$ and thus it is not contained in the line at infinity. The jacobian determinant of $\mathcal{I}$ at a fixed point of $\Gamma$ is -1 hence $\mathcal{I}$ does not preserve $\eta$. 
Contrary to the polynomial case (Proposition 2.2.8) $\operatorname{Bir}\left(\mathbb{C}^{2}\right)_{\eta}$ contains periodic elements that are non-exact. Consider the map $\left(\phi_{0}\left(z_{0}, z_{1}\right), \phi_{1}\left(z_{0}, z_{1}\right)\right)$ where

$$
\phi_{0}\left(z_{0}, z_{1}\right)=-z_{0}+\frac{1}{z_{1}^{2}-1}, \quad \phi_{1}\left(z_{0}, z_{1}\right)=-z_{1} ;
$$

it is a birational involution that preserves $\eta$. Furthermore the 1 -form $\phi_{0} \mathrm{~d} \phi_{1}-z_{0} \mathrm{~d} z_{1}$ has non-trivial residues and so is not exact (Theorem 3.4.1).

We will now focus on quadratic exact birational maps.

Any birational map of $\mathbb{P}^{2}$ can be written as a composition of birational maps of degree $\leq 2$ (see for instance $[\mathbf{1}]$ ). The three following maps are birational and of degree 2

$$
\begin{array}{ll}
\sigma: \mathbb{P}^{2} \rightarrow \mathbb{P}^{2}, & \left(z_{0}: z_{1}: z_{2}\right) \rightarrow\left(z_{1} z_{2}: z_{0} z_{2}: z_{0} z_{1}\right), \\
\rho: \mathbb{P}^{2} \rightarrow \mathbb{P}^{2}, & \left(z_{0}: z_{1}: z_{2}\right) \rightarrow\left(z_{0} z_{2}: z_{0} z_{1}: z_{2}^{2}\right), \\
\tau: \mathbb{P}^{2} \rightarrow \mathbb{P}^{2}, & \left(z_{0}: z_{1}: z_{2}\right) \rightarrow\left(z_{0} z_{2}+z_{1}^{2}: z_{1} z_{2}: z_{2}^{2}\right) .
\end{array}
$$

Denote by $\stackrel{\circ}{B i r}_{2}\left(\mathbb{P}^{2}\right)$ the set of birational maps of $\mathbb{P}^{2}$ of degree 2 exactly; for any $\phi \in$ $\operatorname{Bir}\left(\mathbb{P}^{2}\right)$ set

$$
\mathcal{O}(\phi)=\left\{\mathfrak{g} \phi \mathfrak{h}^{-1} \mid \mathfrak{g}, \mathfrak{h} \in \operatorname{Aut}\left(\mathbb{P}^{2}\right)\right\}
$$

one has $([\mathbf{1 3}])$

$$
\stackrel{\circ}{\operatorname{Bir}}_{2}\left(\mathbb{P}^{2}\right)=\mathcal{O}(\sigma) \cup \mathcal{O}(\rho) \cup \mathcal{O}(\tau) .
$$

Let us now describe the quadratic birational maps that preserve $\eta$; note that $\tau$ preserves $\eta$. Consider $\Upsilon$ the set of pairs $(\mathfrak{g}(\gamma), \mathfrak{g}(\beta))$ where

$$
\mathfrak{g}(\beta)=\left(\frac{\beta_{0} z_{0}+\beta_{1} z_{1}+\beta_{2}}{\beta_{6} z_{0}+\beta_{7} z_{1}+\beta_{8}}, \frac{\beta_{3} z_{0}+\beta_{4} z_{1}+\beta_{5}}{\beta_{6} z_{0}+\beta_{7} z_{1}+\beta_{8}}\right)
$$

in $\operatorname{Aut}\left(\mathbb{P}^{2}\right) \times \operatorname{Aut}\left(\mathbb{P}^{2}\right)$ such that

$$
\gamma_{6}=0, \quad \gamma_{7} \beta_{3}=0, \quad \gamma_{7} \beta_{4}=0, \quad \operatorname{det} \mathfrak{g} \operatorname{det} \mathfrak{h}=\left(\gamma_{7} \beta_{5}+\gamma_{8}\right)^{3} .
$$

Proposition 3.4.5. A quadratic birational map that preserves $\eta$ belongs to $\mathcal{O}(\tau)$. More precisely a birational map belongs to $\operatorname{Bir}_{2}\left(\mathbb{P}^{2}\right) \cap \operatorname{Bir}\left(\mathbb{C}^{2}\right)_{\eta}$ if and only if it can be written $\mathfrak{g}\left(z_{0}+z_{1}^{2}, z_{1}\right) \mathfrak{h}$ with $(\mathfrak{g}, \mathfrak{h})$ in $\Upsilon$. $\mathcal{O}(\rho)$.

Proof. Let $\psi$ be in $\operatorname{Bir}\left(\mathbb{C}^{2}\right)_{\eta} \cap \stackrel{\circ}{\operatorname{Bir}}_{2}\left(\mathbb{P}^{2}\right)$; it is sufficient to prove that $\psi \notin \mathcal{O}(\sigma) \cup$

Assume by contradiction that $\psi$ belongs to $\mathcal{O}(\sigma)$, i.e. $\psi=\mathfrak{g} \sigma \mathfrak{h}$ with $\mathfrak{g}=\mathfrak{g}(\gamma)$, $\mathfrak{h}^{-1}=\mathfrak{g}(\beta)$. One can rewrite $\psi^{*} \eta=\eta$ as $\sigma^{*} \mathfrak{g}^{*} \eta=\mathfrak{h}^{*} \eta$; this last one relation is equivalent in the affine chart $z_{3}=1$ to

$$
\frac{(\operatorname{det} \mathfrak{g}) z_{0} z_{1}}{\left(\gamma_{6} z_{1}+\gamma_{7} z_{0}+\gamma_{8} z_{0} z_{1}\right)^{3}} \eta=\frac{\operatorname{det} \mathfrak{h}}{\left(\beta_{6} z_{0}+\beta_{7} z_{1}+\beta_{8}\right)^{3}} \eta
$$


the coefficients $\gamma_{6}$ and $\gamma_{7}$ have thus to be zero and (3.3) is equivalent to

$$
\frac{\operatorname{det} \mathfrak{g}}{\gamma_{8}^{3} z_{0}^{2} z_{1}^{2}} \eta=\frac{\operatorname{det} \mathfrak{h}}{\left(\beta_{6} z_{0}+\beta_{7} z_{1}+\beta_{8}\right)^{3}} \eta
$$

and this equality never holds.

A similar argument allows to exclude the case: $\psi \in \mathcal{O}(\rho)$. This proves the first assertion.

Let us consider $\psi=\mathfrak{g} \tau \mathfrak{h}$ in $\operatorname{Bir}_{2}\left(\mathbb{P}^{2}\right) \cap \operatorname{Bir}\left(\mathbb{C}^{2}\right)_{\eta}$ with $\mathfrak{g}=\mathfrak{g}(\gamma)$ and $\mathfrak{h}=\mathfrak{g}(\beta)$. The 1-form $\eta$ has a line of poles of order 3 at infinity so does $\psi^{*} \eta$ and so does $\left(z_{0}+z_{1}^{2}, z_{1}\right)^{*} \mathfrak{g}^{*} \eta$. But

$$
\left(z_{0}+z_{1}^{2}, z_{1}\right)^{*} \mathfrak{g}^{*} \eta=\frac{\operatorname{det} \mathfrak{g}}{\left(\gamma_{6}\left(z_{0}+z_{1}^{2}\right)+\gamma_{7} z_{1}+\gamma_{8}\right)^{3}} \eta
$$

therefore $\gamma_{6}$ has to be 0 . This implies that

$$
\psi^{*} \eta=\frac{\operatorname{det} \mathfrak{g} \operatorname{det} \mathfrak{h}}{\left(\gamma_{7}\left(\beta_{3} z_{0}+\beta_{4} z_{1}+\beta_{5}\right)+\gamma_{8}\right)^{3}} \eta
$$

as a consequence $\psi^{*} \eta=\eta$ if and only if

$$
\gamma_{6}=0, \quad \gamma_{7} \beta_{3}=0, \quad \gamma_{7} \beta_{4}=0, \quad \operatorname{det} \mathfrak{g} \operatorname{det} \mathfrak{h}=\left(\gamma_{7} \beta_{5}+\gamma_{8}\right)^{3} .
$$

TheOREM 3.4.6. A generic element of $\operatorname{Bir}_{2}\left(\mathbb{P}^{2}\right) \cap \operatorname{Bir}\left(\mathbb{C}^{2}\right)_{\eta}$ is not exact.

In fact there exists a non-empty Zariski open subset $\widetilde{\Upsilon}$ of $\Upsilon$ such that no element of

$$
\{\mathfrak{g}(\gamma) \tau \mathfrak{g}(\beta) \mid(\mathfrak{g}(\gamma), \mathfrak{g}(\beta)) \in \widetilde{\Upsilon}\}
$$

is exact.

Proof. It is sufficient to exhibit a non-exact element. Let us recall that the birational map $\phi=\left(\phi_{0}, \phi_{1}\right)$ belongs to $\stackrel{\circ}{\operatorname{Bir}}_{2}\left(\mathbb{P}^{2}\right) \cap \operatorname{Bir}\left(\mathbb{C}^{2}\right)_{\eta}$ if and only if it can be written as $\mathfrak{g}(\gamma) \tau \mathfrak{g}(\beta)$ with $(\mathfrak{g}(\gamma), \mathfrak{g}(\beta))$ in $\Upsilon$ (Proposition 3.4.5).

If we consider the special case $\gamma_{i}=\beta_{i}=0$ for any $i \in\{1,2,3,4,6,8\}, \gamma_{5}=\gamma_{7}$ and $\gamma_{0}=\gamma_{7} \beta_{5}^{2} / \beta_{0} \beta_{7}$ then

$$
z_{0} \mathrm{~d} z_{1}-\phi_{0} \mathrm{~d} \phi_{1}=-\frac{\beta_{5}^{2} \mathrm{~d} z_{1}}{\beta_{0} \beta_{7} z_{1}}
$$

But $\operatorname{det} \mathfrak{g}(\beta) \neq 0$ so $\beta_{5} \neq 0$ and $\phi$ can not be lifted to $\operatorname{Bir}\left(\mathbb{C}^{3}\right)_{\omega}$.

The set $\Upsilon$ is rational hence irreducible, this yields the result.

Let us end this section with examples of exact maps.

Proposition 3.4.7. Let $\varphi$ be an automorphism of $\mathbb{P}^{2}$; the map $\varphi$ is exact if and only if $\varphi$ is affine in the affine chart $z_{2}=1$ and preserves $\eta$, that is

$$
\varphi=\left(\delta_{0} z_{0}+\beta_{0} z_{1}+\gamma_{0}, \delta_{1} z_{0}+\beta_{1} z_{1}+\gamma_{1}\right)
$$


with $\delta_{i}, \beta_{i}, \gamma_{i}$ in $\mathbb{C}$ such that $\delta_{0} \beta_{1}-\delta_{1} \beta_{0}=1$.

Proof. The form $\eta$ has a pole at infinity so if $\varphi \in \operatorname{Aut}\left(\mathbb{P}^{2}\right)$ preserves $\eta$, it preserves the pole. Hence $\varphi$ belongs to $\mathrm{Aff}_{2}$, so in particular to $\operatorname{Aut}\left(\mathbb{C}^{2}\right)_{\eta}$ and then $\varphi$ is exact.

We will now consider the subgroup of $\operatorname{Bir}\left(\mathbb{C}^{2}\right)_{\eta}$ that preserves the fibration $z_{0} z_{1}=$ cst fiberwise. The following statement says that this subgroup is not isomorphic to the subgroup of $\operatorname{Bir}\left(\mathbb{C}^{2}\right)_{\eta}$ that preserves $z_{1}=$ cst fiberwise.

Proposition 3.4.8. The set

$$
\Lambda=\left\{\left(z_{0} a\left(z_{0} z_{1}\right), \frac{z_{1}}{a\left(z_{0} z_{1}\right)}\right) \mid a \in \mathbb{C}(t)\right\}
$$

is a subgroup isomorphic to the uncountable abelian subgroup $\left\{\left(a\left(z_{1}\right) z_{0}, z_{1}\right) \mid a \in \mathbb{C}\left(z_{1}\right)^{*}\right\}$ and is contained in $\operatorname{Bir}\left(\mathbb{C}^{2}\right)_{\eta}$.

Any birational map of the form $\left(z_{0} a\left(z_{0}, z_{1}\right), z_{1} / a\left(z_{0}, z_{1}\right)\right)$ that preserves $\eta$ belongs to $\Lambda$.

A generic element of $\Lambda$ is in $\operatorname{Bir}\left(\mathbb{C}^{2}\right)_{\eta}$ but not in im $\varsigma . \quad$ More precisely $\left(z_{0} a\left(z_{0} z_{1}\right), z_{1} / a\left(z_{0} z_{1}\right)\right) \in \Lambda$ is exact if and only if a is a monomial.

If $a$ is a monomial, i.e. $a\left(z_{0} z_{1}\right)=c z_{0}^{\mu} z_{1}^{\mu}$ with $c \in \mathbb{C}^{*}$ and $\mu \in \mathbb{Z}$, then the $\varsigma$-lifted maps are

$$
\left(z_{0} c z_{0}^{\mu} z_{1}^{\mu}, \frac{z_{1}}{c z_{0}^{\mu} z_{1}^{\mu}}, z_{2}-\mu z_{0} z_{1}+\beta\right), \quad \beta \in \mathbb{C}
$$

These maps form a subgroup of $\operatorname{Bir}\left(\mathbb{C}^{3}\right)_{\omega}$ isomorphic to $\mathbb{C} \times \mathbb{C}^{*} \times \mathbb{Z}$.

Proof. The first assertion follows from

$$
\left(z_{0} a\left(z_{0} z_{1}\right), \frac{z_{1}}{a\left(z_{0} z_{1}\right)}\right)=\left(z_{0}, z_{0} z_{1}\right)^{-1}\left(z_{0} a\left(z_{1}\right), z_{1}\right)\left(z_{0}, z_{0} z_{1}\right) .
$$

A direct computation shows that $\Lambda \subset \operatorname{Bir}\left(\mathbb{C}^{2}\right)_{\eta}$.

A birational map $\left(z_{0} a\left(z_{0}, z_{1}\right), z_{1} / a\left(z_{0}, z_{1}\right)\right)$ preserves $\eta$ if and only if

$$
\left(z_{0} \frac{\partial}{\partial z_{0}}-z_{1} \frac{\partial}{\partial z_{1}}\right)(a)=0
$$

that is, if and only if $a=a\left(z_{0} z_{1}\right)$.

Let us consider $\phi=\left(\phi_{0}, \phi_{1}\right)=\left(z_{0} a\left(z_{0} z_{1}\right), z_{1} / a\left(z_{0} z_{1}\right)\right)$ an element of $\Lambda$; then

$$
\phi_{0} \mathrm{~d} \phi_{1}-z_{0} \mathrm{~d} z_{1}=t \frac{a^{\prime}(t)}{a(t)} \mathrm{d} t
$$

with $t=z_{0} z_{1}$. Let us write $a$ as follows:

$$
a(t)=\prod_{i=1}^{n}\left(t-t_{i}\right)^{\mu_{i}}
$$


then

$$
t \frac{a^{\prime}(t)}{a(t)} \mathrm{d} t=t \sum_{i=1}^{n} \frac{\mu_{i}}{t-t_{i}} \mathrm{~d} t
$$

and the residues of this 1 -form are trivial if and only if $a$ is monomial, i.e. $a(t)=c t^{\mu}$ where $c \in \mathbb{C}^{*}$ and $\mu \in \mathbb{Z}$.

We can determine $\mathcal{J} \cap \operatorname{Bir}\left(\mathbb{C}^{2}\right)_{\eta}$ and the exact maps in $\mathcal{J} \cap \operatorname{Bir}\left(\mathbb{C}^{2}\right)_{\eta}$.

Proposition 3.4.9. A Jonquières map of $\mathbb{P}^{2}$ preserves $\eta$ if and only if it can be written as follows

$$
\left(\frac{\left(\gamma z_{1}+\delta\right)^{2}}{\varepsilon \delta-\beta \gamma} z_{0}+r\left(z_{1}\right), \frac{\varepsilon z_{1}+\beta}{\gamma z_{1}+\delta}\right)
$$

where $r$ belongs to $\mathbb{C}\left(z_{1}\right)$ and $\left[\begin{array}{ll}\varepsilon & \beta \\ \gamma & \delta\end{array}\right]$ to $P G L(2 ; \mathbb{C})$.

Furthermore it is exact if it has the following form

$$
\left(\frac{\left(\gamma z_{1}+\delta\right)^{2}}{\varepsilon \delta-\beta \gamma} z_{0}+P\left(z_{1}\right)\left(\gamma z_{1}+\delta\right)^{2}, \frac{\varepsilon z_{1}+\beta}{\gamma z_{1}+\delta}\right)
$$

where $P$ denotes an element of $\mathbb{C}\left[z_{1}\right]$.

Let us now look at monomial maps that belong to $\operatorname{Bir}\left(\mathbb{C}^{2}\right)_{\eta}$ and those who are exact.

Proposition 3.4.10. A monomial map belongs to $\operatorname{Bir}\left(\mathbb{C}^{2}\right)_{\eta}$ if and only if it can be written either

$$
\left(\gamma z_{0}^{p} z_{1}^{p-1}, \frac{1}{\gamma} z_{0}^{1-p} z_{1}^{2-p}\right)
$$

or

$$
\left(\gamma z_{0}^{p} z_{1}^{p+1},-\frac{1}{\gamma} z_{0}^{1-p} z_{1}^{-p}\right)
$$

with $\gamma$ in $\mathbb{C}^{*}$ and $p$ in $\mathbb{Z}$.

Furthermore any monomial map of $\operatorname{Bir}\left(\mathbb{C}^{2}\right)_{\eta}$ is exact.

The s-lifts of a map of type (3.4) are

$$
\left(\gamma z_{0}^{p} z_{1}^{p-1}, \frac{1}{\gamma} z_{0}^{1-p} z_{1}^{2-p}, z_{2}+(p-1) z_{0} z_{1}+\beta\right) \quad \beta \in \mathbb{C}
$$

similarly the $\varsigma$-lifts of a map of type (3.5) are

$$
\left(\gamma z_{0}^{p} z_{1}^{p+1},-\frac{1}{\gamma} z_{0}^{1-p} z_{1}^{-p}, z_{2}+(1-p) z_{0} z_{1}+\beta^{\prime}\right) \quad \beta^{\prime} \in \mathbb{C} .
$$

REMARKS 3.4.11. - Both maps of type (3.4) and of type (3.5) preserve $\left(z_{0} z_{1}\right)^{2}=$ cst. 
- Maps of type (3.4) form a group $\mathrm{G}_{1}$. Note that the matrices $\left[\begin{array}{cc}p & p-1 \\ 1-p & 2-p\end{array}\right]$ are in $S L(2 ; \mathbb{Z})$; they are stochastic up to transposition and have trace equal to 2 . The group

$$
\left\{\left[\begin{array}{rr}
p & p-1 \\
1-p 2-p
\end{array}\right] \mid p \in \mathbb{Z}\right\}
$$

is isomophic to $\mathbb{Z}$. As a consequence $\mathrm{G}_{1}$ is isomorphic to $\mathbb{C}^{*} \times \mathbb{Z}$.

The maps of type (3.5) don't form a group. The corresponding matrices $\left[\begin{array}{cc}p & p+1 \\ 1-p & -p\end{array}\right]$ have determinant -1 , trace 0 and are stochastic up to transposition.

But the union of the maps of type (3.4) or (3.5) is a group which is a double extension of $\mathbb{C}^{*} \times \mathbb{Z}$.

\subsection{Indeterminacy and exceptional sets.}

As we have seen if $\phi$ is a contact map, then $\mathcal{H}_{\infty}$ is either preserved by $\phi$, or blown down by $\phi$ (Proposition 3.3.1). In case it is blown down, $\mathcal{H}_{\infty}$ can be blown down onto a point or onto a curve; in this last eventuality $\mathcal{H}_{\infty}$ can be contracted onto a curve contained in $\mathcal{H}_{\infty}$ (take for instance $\left.\phi=\mathcal{K}\left(z_{1}, z_{1} z_{2}\right)\right)$. Note also that $\mathcal{H}_{\infty}$ can be contracted onto a curve not contained in $\mathcal{H}_{\infty}$ : the map $\mathcal{K}\left(z_{1} / z_{2}, 1 / z_{2}\right)$ blows down $\mathcal{H}_{\infty}$ onto the legendrian curve $z_{0}=z_{2}=0$. We will see that this is a general case and for any contracted surface:

Proposition 3.5.1. Let $\phi$ be a contact birational map of $\mathbb{P}^{3}$. Assume that $\phi$ blows down a surface $\mathcal{S}$ onto a curve $\mathcal{C}$. Then

- either $\mathcal{C}$ is contained in $\mathcal{H}_{\infty}$,

- $\operatorname{or} \mathcal{C}$ is an algebraic legendrian curve.

Corollary 3.5.2. Let $\phi$ be a contact birational map of $\mathbb{P}^{3}$. If $\mathcal{C}$ is a curve not contained in $\mathcal{H}_{\infty}$ and blown-up by $\phi$ on a surface distinct from $\mathcal{H}_{\infty}$, then $\mathcal{C}$ is a legendrian curve.

Let us now give an example of maps of finite order that illustrates Proposition 3.5.6.

ExAMPLE 3.5.3. Start with the birational map $\varphi=\left(z_{2},\left(z_{2}+1\right) / z_{1}\right)$ of order 5 . The map $\mathcal{K}(\varphi)=\left(-\left(z_{2}+1+z_{0} z_{1}\right) / z_{0} z_{1}^{2}, z_{2},\left(z_{2}+1\right) / z_{1}\right)$ blows down $z_{2}=-z_{3}$ onto the legendrian curve $\left(z_{2}=z_{1}+z_{3}=0\right)$;

Proof of Proposition 3.5.1. We will distinguish the cases $\mathcal{S}=\mathcal{H}_{\infty}$ and $\mathcal{S} \neq$ $\mathcal{H}_{\infty}$

Let us start with the eventuality $\mathcal{S}=\mathcal{H}_{\infty}$. Suppose that $\mathcal{C}$ is not contained in $\mathcal{H}_{\infty}$. Note that $\phi_{\mid \mathcal{H}_{\infty} \backslash \text { Ind } \phi}$ is holomorphic of rank $\leq 1$. If $p$ belongs to $\mathcal{C} \backslash \operatorname{Ind} \phi$, then $\phi^{-1}(p)$ is a curve contained in $\mathcal{H}_{\infty}$; there exists a curve $\mathcal{C}^{\prime}$ transverse to

$$
\left\{\phi^{-1}(p) \mid p \in \mathcal{C} \backslash \operatorname{Ind} \phi\right\}
$$

contained in $\mathcal{H}_{\infty}$ and such that $\phi\left(\mathcal{C}^{\prime}\right)=\mathcal{C}$. Consider a parametrization $s$ of $\mathcal{C}^{\prime}$; then $t=\phi \circ s$ is a parametrization of $\mathcal{C}$ and 


$$
t^{*} \omega=(\phi \circ s)^{*} \omega=s^{*} \phi^{*} \omega=s^{*} V(\phi) \omega=V(\phi) \circ s \cdot s^{*} \omega=0 .
$$

Assume now that $\mathcal{S} \neq \mathcal{H}_{\infty}$ and $\mathcal{C} \not \subset \mathcal{H}_{\infty}$. Set $\mathcal{C}=\phi(\mathcal{S})$. Let us consider a generic point $p$ of $\mathcal{S}$. The germ $\phi_{, p}$ is holomorphic and $\phi(p) \in \mathcal{C}$ does not belong to $\mathcal{H}_{\infty}$. In particular the 3-form $\phi^{*} \omega \wedge \mathrm{d} \omega$ is thus holomorphic at $p$; in fact $V(\phi)_{, p}$ is holomorphic and as we have seen

$$
\phi^{*} \omega \wedge \mathrm{d} \omega=V(\phi)^{2} \omega \wedge \mathrm{d} \omega .
$$

Since $\mathcal{S}$ is blown down by $\phi$, the jacobian determinant of $\phi$ is identically zero on $\mathcal{S}$ and then $V(\phi)$ vanishes on $\mathcal{S}$.

Assume that $\mathcal{C}$ is not a legendrian curve, then the restriction of $\omega$ to $\mathcal{C}$ in a neighborhood of $\phi(p)$ defines a 1-form $\Theta$ on $\mathcal{C}$ without zero (let us recall that $p$ is generic). As the restriction

$$
\phi_{,\left.p\right|_{\mathcal{S}, p}}: \mathcal{S}_{, p} \rightarrow \mathcal{C}_{, \phi(p)}
$$

is locally a submersion, $\phi_{,\left.p\right|_{\mathcal{S}, p} ^{*}} \Theta$ is a nonzero 1 -form on $\mathcal{S}_{, p}$ : contradiction with the fact that $\phi_{, p}^{*} \omega$ vanishes on $\mathcal{S}_{, p}$.

There is no statement if $\phi \in \operatorname{Bir}\left(\mathbb{C}^{3}\right)_{\mathrm{c}(\omega)}$ blows down $\mathcal{H}_{\infty}$ onto a point. Indeed

$$
\mathcal{K}\left(\frac{z_{1}}{z_{2}^{2}}, \frac{z_{1}}{z_{2}^{3}}\right)=\left(\frac{z_{2}+3 z_{0} z_{1}}{z_{2}\left(z_{2}-2 z_{0} z_{1}\right)}, \frac{z_{1}}{z_{2}^{2}}, \frac{z_{1}}{z_{2}^{3}}\right)
$$

contracts $\mathcal{H}_{\infty}$ onto $\boldsymbol{e}_{3} \notin \mathcal{H}_{\infty}$ but $\mathcal{K}\left(z_{1} z_{2}, z_{1} z_{2}^{2}\right)$ contracts $\mathcal{H}_{\infty}$ onto $\boldsymbol{e}_{2} \in \mathcal{H}_{\infty}$. But we get some result when $\phi \in \operatorname{Bir}\left(\mathbb{C}^{3}\right)_{\mathrm{c}(\omega)}$ blows down a surface distinct from $\mathcal{H}_{\infty}$ onto a point.

Definition. Let $\phi$ be a contact birational map of $\mathbb{P}^{3}$. Let $\mathcal{S}=(f=0)$ be an irreducible surface blown down by $\phi$, and let $p$ be a smooth point of $\mathcal{S}$ such that $\phi$ and $V(\phi)$ are holomorphic at $p$. The multiplicity of contraction of $\phi$ at $p$ is the greatest integer $n$ such that $f_{, p}^{n}$ divides $V(\phi)$. One can check that $n$ is independent on $p$. The integer $n$ is the multiplicity of contraction of $\phi$ on $\mathcal{S}$.

REMARK 3.5.4. Let $\phi$ be a contact birational map of $\mathbb{P}^{3}$. If $\phi$ is holomorphic at $p \in \mathbb{P}^{3} \backslash \mathcal{H}_{\infty}$, then $V(\phi)$ is too.

EXAMPLE 3.5.5. Let us consider the birational map $\phi$ defined in the affine chart $z_{1}=1$ by

$$
\phi=\left(\frac{z_{0} z_{3}^{2}}{\left(z_{2}+z_{3}\right)^{2}}, \frac{z_{2} z_{3}}{\left(z_{2}+z_{3}\right)}, z_{3}\right)
$$

in this chart $\omega=\mathrm{d} z_{2}-\left(z_{0}+z_{2} z_{3}\right) / z_{3}^{2} \mathrm{~d} z_{3}$ and one can check that $V(\phi)=z_{3}^{2} /\left(z_{2}+z_{3}^{2}\right)$. Furthermore $\mathcal{H}_{\infty}$ is blown down by $\phi$ onto the point $(0,0,0)$; the multiplicity of contraction of $\phi$ on $\mathcal{H}_{\infty}$ is thus 2 . 
Proposition 3.5.6. Let $\phi$ be a map of $\operatorname{Bir}\left(\mathbb{C}^{3}\right)_{\mathrm{c}(\omega)}$ and let $\mathcal{S}$ be an irreducible surface distinct from $\mathcal{H}_{\infty}$ blown down by $\phi$ onto a point $p$. If the multiplicity of contraction of $\phi$ on $\mathcal{S}$ is 1 , then $p$ belongs to $\mathcal{H}_{\infty}$.

REMARK 3.5.7. As soon as the multiplicity of contraction of $\phi$ on $\mathcal{S}$ is $>1$, the point $p$ can be in $\mathbb{P}^{3} \backslash \mathcal{H}_{\infty}$. Let us consider the map of $\operatorname{Bir}\left(\mathbb{C}^{3}\right)_{\mathrm{c}(\omega)}$ given in the affine chart $z_{3}=1$ by

$$
\left(\frac{z_{2}\left(n z_{0} z_{1}-z_{2}\right)}{z_{2}+(1-n) z_{0} z_{1}}, z_{1} z_{2}^{n-1}, z_{1} z_{2}^{n}\right)
$$

with $n \in \mathbb{Z}$. The surface $z_{2}=0$ is blown down onto $\boldsymbol{e}_{3} \notin \mathcal{H}_{\infty}$. One can check that $V(\phi)=z_{1} z_{2}^{n} /\left(z_{2}+(1-n) z_{0} z_{1}\right)$ so the multiplicity of contraction of $\phi$ on $z_{2}=0$ is $n$ if $n \geq 2$ and 0 otherwise.

Proof of Proposition 3.5.6. Assume by contradiction that $p=\left(p_{0}, p_{1}, p_{2}\right)$ does not belong to $\mathcal{H}_{\infty}$. Let $(f=0)$ be an equation of $\mathcal{S}$; as the multiplicity of contraction of $\phi$ on $\mathcal{S}$ is 1 one has $V(\phi)=f V_{1}$ with $V_{1 \mid \mathcal{S}}$ generically regular. There exists a point $m \in \mathcal{S}$ such that $f_{, m}$ is a submersion and $\phi$ is holomorphic at $m$. One has $\phi_{, m}=\left(p_{0}+f A, p_{1}+f B, p_{2}+f C\right)$ with $A, B, C$ holomorphic and $\phi_{, m}^{*} \omega=V(\phi) \omega$ can be rewritten

$$
\left(f A+p_{0}\right)(f \mathrm{~d} B+B \mathrm{~d} f)+(f \mathrm{~d} C+C \mathrm{~d} f)=f V_{1}\left(z_{0} \mathrm{~d} z_{1}+\mathrm{d} z_{2}\right) .
$$

This implies that there exists $C_{1}$ holomorphic such that $p_{0} B+C=f C_{1}$, i.e. $C=$ $f C_{1}-p_{0} B$. Hence

$$
(3.6) \Longleftrightarrow f A \mathrm{~d} B+A B \mathrm{~d} f+f \mathrm{~d} C_{1}+2 C_{1} \mathrm{~d} f=V_{1}\left(z_{0} \mathrm{~d} z_{1}+\mathrm{d} z_{2}\right) .
$$

The multiplicity of contraction of $\phi$ on $\mathcal{S}$ is 1 hence $f$ does not divide $V_{1}$. Then $\mathcal{S}$ is invariant by $\omega$ and this gives a contradiction with the fact that $\mathcal{H}_{\infty}$ is the only invariant surface of $\omega$.

For elements in $\operatorname{Bir}\left(\mathbb{C}^{3}\right)_{\omega}$ we only have one statement that includes both cases of a surface contracted onto a point and onto a curve. Let us remark that in the case of a point, we don't need the assumption about the multiplicity of contraction; in the other one the statement shows that Proposition 3.5.1 applies to elements of $\operatorname{Bir}\left(\mathbb{C}^{3}\right)_{c}(\omega) \backslash \operatorname{Bir}\left(\mathbb{C}^{3}\right)_{\omega}$.

Proposition 3.5.8. Let $\phi$ be a map of $\operatorname{Bir}\left(\mathbb{C}^{3}\right)_{\omega}$. If $\mathcal{S}$ is a surface distinct from $\mathcal{H}_{\infty}$ contracted by $\phi$, then $\phi(\mathcal{S})$ belongs to $\mathcal{H}_{\infty}$.

Proof. From $\phi^{*} \omega=\omega$ one gets $\phi^{*}(\omega \wedge \mathrm{d} \omega)=\omega \wedge \mathrm{d} \omega=\mathrm{d} z_{0} \wedge \mathrm{d} z_{1} \wedge \mathrm{d} z_{2}$. Suppose that for $p \in \mathcal{S}$ generic $\phi(p)$ does not belong to $\mathcal{H}_{\infty}$. As codim Ind $\phi \geq 2$, the map $\phi$ is holomorphic at $p$. Since $\phi$ preserves the volume form, $\phi$ is a diffeomorphism; hence $\phi$ cannot blow down a subvariety onto a curve or a point not contained in $\mathcal{H}_{\infty}$.

EXAMPLE 3.5.9. If $\phi=\left(\phi_{1}, \phi_{2}\right)=\left(z_{1}^{p} z_{2}^{q}, z_{1}^{r} z_{2}^{s}\right)$, with $\left[\begin{array}{cc}p & q \\ r & s\end{array}\right] \in S L(2 ; \mathbb{Z})$, then 


$$
\mathcal{K}(\phi)=\left(z_{1}^{r-p} z_{2}^{s-q} \frac{-r z_{2}+s z_{0} z_{1}}{p z_{2}-q z_{0} z_{1}}, z_{1}^{p} z_{2}^{q}, z_{1}^{r} z_{2}^{s}\right) .
$$

Note that for any $\left[\begin{array}{ll}p & q \\ r & s\end{array}\right] \in S L(2 ; \mathbb{Z})$ the map $\mathcal{K}(\phi)$ belongs to $\operatorname{Bir}\left(\mathbb{C}^{3}\right)_{\mathrm{c}(\omega)} \backslash \operatorname{Bir}\left(\mathbb{C}^{3}\right)_{\omega}$.

For instance if $\left[\begin{array}{ll}p & q \\ r & s\end{array}\right]=\left[\begin{array}{cc}-1 & 0 \\ 0 & -1\end{array}\right]$, i.e. if $\sigma=\left(1 / z_{0}, 1 / z_{1}\right)$ is the Cremona involution, then

$$
\mathcal{K}(\sigma)=\mathcal{K}\left(\sigma^{-1}\right)=\left(\frac{z_{0} z_{1}^{2}}{z_{2}^{2}}, \frac{1}{z_{1}}, \frac{1}{z_{2}}\right)
$$

and Ind $\mathcal{K}(\sigma)=\left\{z_{0}=z_{2}=0\right\} \cup\left\{z_{0}=z_{3}=0\right\} \cup\left\{z_{1}=z_{2}=0\right\} \cup\left\{z_{1}=z_{3}=0\right\}$; furthermore $z_{2}=0$ and $\mathcal{H}_{\infty}$ are blown down onto $\boldsymbol{e}_{1}$ and $z_{1}=0$ onto $\boldsymbol{e}_{2}$.

\section{Some common properties.}

\subsection{Invariant curves and surfaces.}

The following statement is a local statement of contact analytic geometry.

Proposition 4.1.1. Let $\phi$ be an element of $\operatorname{Aut}\left(\mathbb{C}^{3}\right)_{\omega}$ or $\operatorname{Bir}\left(\mathbb{C}^{3}\right)_{\omega}$. Suppose that $m$ is a periodic point of $\phi$ and that there exists a germ of irreducible curve $\mathcal{C}$ invariant by $\phi$, passing through $m$. Then

- either $\mathcal{C}$ is a curve of periodic points (i.e. $\phi_{\mid \mathcal{C}}^{\ell}=\mathrm{id}$ for some integer $\ell$ ),

- or $\mathcal{C}$ is a legendrian curve.

Let us note that according to Proposition 4.2.4 we know that such a situation often occurs.

Proof. Assume that $\phi$ belongs to Aut $\left(\mathbb{C}^{3}\right)_{\omega}$. Up to considering a well-chosen iterate of $\phi$ let us assume that $m$ is a fixed point of $\phi$. Let $s \mapsto \gamma(s)$ be a local parametrization of $\mathcal{C}$ at $m$. Up to reparametrization one can suppose that $\gamma(0)=m$. Let $\varphi$ be the "restriction" to $\mathcal{C}$ of $\phi$, that is the local map $\varphi: \mathbb{C}_{, 0} \rightarrow \mathbb{C}$ defined by $\varphi(0)=0$ and

$$
\forall s \in \mathbb{C}_{, 0} \quad \phi(\gamma(s))=\gamma(\varphi(s)) .
$$

On the one hand $\gamma^{*} \omega=\varepsilon(s) \mathrm{d} s$ and on the other hand $\gamma^{*} \omega=\gamma^{*} \phi^{*} \omega=(\phi \circ \gamma)^{*} \omega$ so

$$
\varepsilon(s) \mathrm{d} s=\varphi^{*}(\varepsilon(s) \mathrm{d} s)=\varepsilon(\varphi) \varphi^{\prime} \mathrm{d} s .
$$

Let us set $\widetilde{\varepsilon}(s)=\int_{0}^{s} \varepsilon(t) \mathrm{d} t$. One has $(\widetilde{\varepsilon}(\varphi))^{\prime}=\varepsilon(\varphi) \varphi^{\prime}=\varepsilon(s)=(\widetilde{\varepsilon}(s))^{\prime}$ hence $\widetilde{\varepsilon}(\varphi)=\widetilde{\varepsilon}+\beta$ for some $\beta \in \mathbb{C}$. As $\varphi(0)=0$, one gets $\beta=0$ and $\widetilde{\varepsilon}(\varphi)=\widetilde{\varepsilon}$. Then:

- either $\widetilde{\varepsilon}=0$ therefore $\varepsilon=0$ and $\mathcal{C}$ is a legendrian curve.

- or there exists some local coordinate for which $\widetilde{\varepsilon}=z^{\ell}, \varphi=e^{2 \mathrm{i} \pi k / \ell} z$ and $\phi_{\mid \mathcal{C}}^{\ell}=$ id.

If $\varphi$ is a polynomial automorphism of $\mathbb{C}^{2}$ that preserves a curve distinct from the line at infinity, then $\varphi$ is conjugate to a Jonquières polynomial automorphism $([\mathbf{8}])$; in particular $\varphi$ preserves a rational fibration. We have a similar statement in dimension 3 : 
Proposition 4.1.2. If $\phi \in \operatorname{Aut}\left(\mathbb{C}^{3}\right)_{\omega}$ preserves a surface, then

$$
\phi=\left(\varphi\left(z_{0}, z_{1}\right), z_{2}+b\left(z_{0}, z_{1}\right)\right)
$$

where $\varphi$ is $\operatorname{Aut}\left(\mathbb{C}^{2}\right)$-conjugate to a Jonquières polynomial automorphism.

Proof. Let us write $\phi$ as $\left(\phi_{0}\left(z_{0}, z_{1}\right), \phi_{1}\left(z_{0}, z_{1}\right), z_{2}+b\left(z_{0}, z_{1}\right)\right)$ and set $\varphi=\left(\phi_{0}, \phi_{1}\right)$.

First note that if $b \equiv 0$ then $\phi_{0} \mathrm{~d} \phi_{1}-z_{0} \mathrm{~d} z_{1}=0$; as a result $\phi_{1}=\phi_{1}\left(z_{1}\right)$ and $\varphi$ is a Jonquières polynomial automorphism.

Let us now assume that the surface $\mathcal{S}$ preserved by $\phi$ is described by

$$
a_{\ell}\left(z_{0}, z_{1}\right) z_{2}^{\ell}+a_{\ell-1}\left(z_{0}, z_{1}\right) z_{2}^{\ell-1}+a_{\ell-2}\left(z_{0}, z_{1}\right) z_{2}^{\ell-2}+\cdots=0
$$

where $a_{i} \in \mathbb{C}\left[z_{0}, z_{1}\right]$, or equivalently by

$$
z_{2}^{\ell}+\widetilde{a}_{\ell-1}\left(z_{0}, z_{1}\right) z_{2}^{\ell-1}+\widetilde{a}_{\ell-2}\left(z_{0}, z_{1}\right) z_{2}^{\ell-2}+\cdots=0
$$

where $\widetilde{a}_{i}=a_{i} / a_{\ell}$. Writing that $\mathcal{S}$ is invariant by $\phi$ one gets that

$$
\begin{aligned}
& \left(z_{2}+b\left(z_{0}, z_{1}\right)\right)^{\ell}+\widetilde{a}_{\ell-1}\left(\varphi\left(z_{0}, z_{1}\right)\right)\left(z_{2}+b\left(z_{0}, z_{1}\right)\right)^{\ell-1} \\
& +\widetilde{a}_{\ell-2}\left(\varphi\left(z_{0}, z_{1}\right)\right)\left(z_{2}+b\left(z_{0}, z_{1}\right)\right)^{\ell-2}+\cdots \\
& \quad=z_{2}^{\ell}+\widetilde{a}_{\ell-1}\left(z_{0}, z_{1}\right) z_{2}^{\ell-1}+\widetilde{a}_{\ell-2}\left(z_{0}, z_{1}\right) z_{2}^{\ell-2}+\cdots
\end{aligned}
$$

Looking at terms in $z_{2}^{\ell-1}$ one gets that $\ell b\left(z_{0}, z_{1}\right)=\widetilde{a}_{\ell-1}\left(z_{0}, z_{1}\right)-\widetilde{a}_{\ell-1}\left(\varphi\left(z_{0}, z_{1}\right)\right)$.

- If $\widetilde{a}_{\ell-1}$ is constant, then $b \equiv 0$ and as we just see $\varphi$ is a Jonquières polynomial automorphism.

- Otherwise $\phi$ is conjugate $\left(\operatorname{in} \operatorname{Bir}\left(\mathbb{P}^{3}\right)\right)$ via $\left(z_{0}, z_{1}, z_{2}+\widetilde{a}_{\ell-1} / \ell\right)$ to $\psi=\left(\varphi, z_{2}\right)$. The map $\psi$ preserves $\widetilde{\omega}=z_{0} \mathrm{~d} z_{1}+\mathrm{d}\left(z_{2}+\widetilde{a}_{\ell-1} / \ell\right)$, the surface $\widetilde{\mathcal{S}}$ given by

$$
z_{2}^{\ell}+\widetilde{a}_{\ell-2}\left(z_{0}, z_{1}\right) z_{2}^{\ell-2}+\widetilde{a}_{\ell-3}\left(z_{0}, z_{1}\right) z_{2}^{\ell-3}+\cdots=0
$$

and thus $\widetilde{a}_{i}(\varphi)=\widetilde{a}_{i}$. If one of the $\widetilde{a}_{i}$ is non-constant, then $\varphi$ is a Jonquières polynomial automorphism. Otherwise $\widetilde{\mathcal{S}}=\cup_{j}\left(z_{2}=c_{j}\right)$; up to take an iterate $\psi^{k}$ of $\psi$ one can suppose that any $z_{2}=c_{j}$ is invariant. Consider $z_{2}=c_{0}$; up to a well-chosen translation (that belongs to $\operatorname{Bir}\left(\mathbb{C}^{3}\right)_{\omega}$ ) the hypersurface $z_{2}=0$ is invariant, that is $\psi^{k}$ is a Jonquières map and so does $\psi$.

EXAMPLE 4.1.3. For any $n \geq 1$ consider $\phi=\left(z_{0}+z_{1}^{n}, z_{1}, z_{2}-z_{1}^{n+1} /(n+1)\right)$ in $\operatorname{Aut}\left(\mathbb{C}^{3}\right)_{\omega}$. The map $\varphi=\left(z_{0}+z_{1}^{n}, z_{1}\right)$ is a Jonquières polynomial automorphism. The surface $\mathcal{S}$ given by $z_{2}+z_{0} z_{1} /(n+1)=0$, is invariant by $\phi$. The foliation induced by $\omega$ on $\mathcal{S}$ is described by the linear differential equation $n z_{0} \mathrm{~d} z_{1}-z_{1} \mathrm{~d} z_{0}$. In fact the functions $z_{2}+z_{0} z_{1} /(n+1)$ and $z_{1}$ are invariant by $\phi$ and the commutative Lie algebra generated by the vector fields $\partial / \partial z_{0}+z_{1} /(n+1) \cdot \partial / \partial z_{2}$ and $\partial / \partial z_{2}$ are invariant by $\phi$.

In general an element of Aut $\left(\mathbb{C}^{3}\right)_{\omega}$ has no invariant surface. For instance there is no polynomial solution to 


$$
-a\left(\varphi\left(z_{0}, z_{1}\right)\right)+a\left(z_{0}, z_{1}\right)=-\frac{z_{1}^{n+1}}{n+1}+\beta
$$

with $\varphi=\left(z_{0}+z_{1}^{n}, z_{1}\right)$ as soon as $\beta \neq 0$.

REMARK 4.1.4. If $\phi \in \operatorname{Bir}\left(\mathbb{C}^{3}\right)_{\omega}$ preserves $z_{2}=0$, then $\phi$ belongs to the Klein family; more precisely $\phi=\left(z_{0} / \nu^{\prime}\left(z_{1}\right), \nu\left(z_{1}\right), z_{2}\right)$ with $\nu \in P G L\left(2 ; \mathbb{C}\left(z_{1}\right)\right)$. Indeed since $\phi$ belongs to $\operatorname{Bir}\left(\mathbb{C}^{3}\right)_{\omega}$,

$$
\phi=\left(\phi_{0}\left(z_{0}, z_{1}\right), \phi_{1}\left(z_{0}, z_{1}\right), z_{2}+b\left(z_{0}, z_{1}\right)\right) .
$$

But $\phi$ preserves $z_{2}=0$ so $b \equiv 0$ and $\phi^{*} \omega=\omega$ implies that $\phi_{1}=\nu\left(z_{1}\right)$ with $\nu \in$ $P G L\left(2 ; \mathbb{C}\left(z_{1}\right)\right)$ and $\phi_{0}=z_{0} / \nu^{\prime}\left(z_{1}\right)$.

Of course there are more general contact maps that preserve $z_{2}=0$; let us give some examples:

$$
\mathcal{K}\left(z_{1}, \frac{z_{2}}{a\left(z_{1}\right) z_{2}+1}\right), \quad \mathcal{K}\left(z_{1}+P\left(z_{2}\right), z_{2}\right)
$$

where $a \in \mathbb{C}\left(z_{1}\right)^{*}$ and $P \in \mathbb{C}\left[z_{2}\right]$.

Let $\phi$ be an element of $\operatorname{Bir}\left(\mathbb{C}^{3}\right)_{\omega}$. Suppose that $\phi$ preserves a surface $\mathcal{S}$ distinct from $\mathcal{H}_{\infty}$. The contact form is non-zero on $\mathcal{S}$ so induces a foliation $\mathcal{F}$ on $\mathcal{S}$, necessarily invariant by $\phi$; let us describe $\left(\mathcal{S}, \phi_{\mid \mathcal{S}}, \mathcal{F}\right)$ :

Proposition 4.1.5. Let $\phi$ be an element of $\operatorname{Bir}\left(\mathbb{C}^{3}\right)_{\omega}$ that preserves a surface distinct from $\mathcal{H}_{\infty}$. Then $\phi$ is $\operatorname{Bir}\left(\mathbb{P}^{3}\right)$-conjugate to $\left(\varphi\left(z_{0}, z_{1}\right), z_{2}\right)$ with $\varphi$ in $\operatorname{Bir}\left(\mathbb{P}^{2}\right)$. The map $\varphi$ preserves a codimension 1 foliation given by a closed 1 -form. As a consequence $\phi$ preserves a "vertical" foliation and a rational function $z_{2}+a\left(z_{0}, z_{1}\right)$.

Proof. Let us denote by $\mathcal{S}$ the surface invariant by $\phi=\left(\varphi\left(z_{0}, z_{1}\right), z_{2}+b\left(z_{0}, z_{1}\right)\right)$ with $\varphi \in \operatorname{Bir}\left(\mathbb{P}^{2}\right)$. One can assume that $\mathcal{S}$ is given by

$$
z_{2}^{\ell}+a_{\ell-1}\left(z_{0}, z_{1}\right) z_{2}^{\ell-1}+\cdots=0 .
$$

The fact that $\mathcal{S}$ is invariant by $\phi$ implies that $a_{\ell-1}\left(z_{0}, z_{1}\right)-a_{\ell-1}\left(\varphi\left(z_{0}, z_{1}\right)\right)=\ell b\left(z_{0}, z_{1}\right)$. Let us consider the map $\psi=\left(z_{0}, z_{1}, z_{2}+\left(a_{\ell-1}\left(z_{0}, z_{1}\right)\right) / \ell\right)$. One has

$$
\begin{aligned}
\widetilde{\phi} & =\psi \phi \psi^{-1}=\left(\varphi\left(z_{0}, z_{1}\right), z_{2}+b\left(z_{0}, z_{1}\right)-\frac{a_{\ell-1}\left(z_{0}, z_{1}\right)}{\ell}+\frac{a_{\ell-1}\left(\varphi\left(z_{0}, z_{1}\right)\right)}{\ell}\right) \\
& =\left(\varphi\left(z_{0}, z_{1}\right), z_{2}\right) .
\end{aligned}
$$

As $\mathcal{S}$ and $\omega$ are invariant by $\phi$, the restriction $\phi_{\mid \mathcal{S}}$ preserves the foliation induced by $\omega$ on $\mathcal{S}$, and $\widetilde{\phi}$ preserves the "vertical" foliation given by $z_{0} \mathrm{~d} z_{1}-\mathrm{d} a_{\ell-1}\left(z_{0}, z_{1}\right)$. Therefore $\varphi$ preserves a codimension 1 foliation given by a closed 1 -form.

EXAMPLE 4.1.6. If $\phi=\left(z_{2}, z_{1} z_{2}^{n}\right)$, then $\mathcal{K}(\phi)=\left(-\left(z_{2}^{n} / z_{0}\right)+n z_{1}, z_{1} z_{2}^{n}, z_{2}\right)$ belongs to $\operatorname{Bir}\left(\mathbb{C}^{3}\right)_{\mathrm{c}(\omega)} \backslash \operatorname{Bir}\left(\mathbb{C}^{3}\right)_{\omega}$, preserves the surface $z_{1}=0$ and also $z_{2}=$ cst. 


\subsection{Dynamical properties.}

Let us first focus on periodic points.

Let $\phi$ be a birational map of $\mathbb{P}^{n}$; a point $p$ is a periodic point of $\phi$ of period $\ell$ if $\phi$ is holomorphic on a neighborhood of any point of $\left\{\phi^{j}(q) \mid j=0, \ldots, \ell-1\right\}$ and if $\phi^{\ell}(q)=q$ and $\phi^{j}(q) \neq q$ for $1 \leq j \leq \ell-1$.

Recall that a polynomial automorphism of $\mathbb{C}^{2}$ of Hénon type (see [19]) has an infinite number of hyperbolic periodic points. For any of these points $p$ of period $\ell_{p}$ there exists a stable manifold $\mathrm{W}^{s}(p)$ defined as the set of points that move towards the orbit of $p$ by positive iteration of $\varphi^{\ell_{p}}$; such a $\mathrm{W}^{s}(p)$ is an immersion from $\mathbb{C}$ to $\mathbb{C}^{2}$. Remark that even if $\mathrm{W}^{s}(m) \neq \mathrm{W}^{s}(p)$ are different as soon as $p$ and $m$ have distinct orbits one has $\overline{\mathrm{W}^{s}(m)}=\overline{\mathrm{W}^{s}(p)}$. The Julia set of $\varphi$ is the topological boundary of the set of points with bounded positive orbits. One can prove that the Julia set of $\varphi$ is equal to the closure of any of the stable manifold. Hence its topology is very complicated: this set contains an infinite number of immersions of $\mathbb{C}$ and pairwise distinct ([19]).

EXAMPLE 4.2.1. Let us consider a polynomial automorphism $\varphi$ of Hénon type given by $\varphi=\left(\beta z_{1}+z_{0}^{2},-\gamma z_{0}\right)$. A $\varsigma$-lift of $\varphi$ to $\operatorname{Aut}\left(\mathbb{C}^{3}\right)_{\mathrm{c}(\omega)}$ is

$$
\phi=\left(\beta z_{1}+z_{0}^{2},-\gamma z_{0}, \gamma \beta z_{2}+\gamma \beta z_{0} z_{1}+\frac{\gamma}{3} z_{0}^{3}\right) .
$$

Take a periodic point $\left(p_{0}, p_{1}\right)$ of $\varphi$ of period $k$; then as $\phi^{k}=\left(\varphi^{k}\left(z_{0}, z_{1}\right),(\gamma \beta)^{k} z_{2}+\right.$ $\left.f\left(z_{0}, z_{1}\right)\right)$ one gets, as soon as $\gamma \beta$ is not a root of unity, that there exists $p_{2}$ such that $\phi^{k}\left(p_{0}, p_{1}, p_{2}\right)=\left(p_{0}, p_{1}, p_{2}\right)$.

More generally, one can state:

Proposition 4.2.2. Let $\phi$ the element of $\operatorname{Bir}\left(\mathbb{C}^{3}\right)_{\mathrm{c}(\omega)}$ of the following type

$$
\phi=\left(\varphi, \operatorname{det} \operatorname{jac} \varphi z_{2}+b\left(z_{0}, z_{1}\right)\right)
$$

with $\varphi$ in $\operatorname{Bir}\left(\mathbb{P}^{2}\right)$ and $b$ in $\mathbb{C}\left(z_{0}, z_{1}\right)$.

If det jac $\varphi$ is not a root of unity, then any periodic point of $\varphi$ can be lifted into a periodic point of $\phi$.

Corollary 4.2.3. Let $\varphi$ be a polynomial automorphism of $\mathbb{C}^{2}$ of Hénon type. A $\varsigma$-lift of $\varphi$ has an infinite number of periodic points that lift the hyperbolic periodic points of $\varphi$.

Question 3. Let $\varphi$ be a Hénon automorphism and let $\phi$ be a $\varsigma$-lift of $\varphi$. The closure of the hyperbolic periodic points of $\varphi$ is the Julia set of $\varphi$; in particular it is a Cantor set. Is the closure of the set of periodic points of $\phi$ a Cantor set?

Let us consider a Hénon automorphism $\varphi=\left(\varphi_{1}, \varphi_{2}\right)$ and let $m$ be an hyperbolic periodic point of $\varphi$; then the matrix 


$$
\left[\begin{array}{cc}
-\frac{\partial \varphi_{2}}{\partial z_{1}} & \frac{\partial \varphi_{2}}{\partial z_{2}} \\
\frac{\partial \varphi_{1}}{\partial z_{1}} & -\frac{\partial \varphi_{1}}{\partial z_{2}}
\end{array}\right]
$$

is a non-parabolic one and so $z_{0} \mapsto\left(-\partial \varphi_{2} / \partial z_{1}+\partial \varphi_{2} / \partial z_{2} z_{0}\right) /\left(\partial \varphi_{1} / \partial z_{1}-\partial \varphi_{1} / \partial z_{2} z_{0}\right)$ has two fixed points. We can thus state the following:

Proposition 4.2.4. Let $\varphi$ be an automorphism of $\mathbb{C}^{2}$ of Hénon type; to any periodic point of period $\ell$ of $\varphi$ corresponds two periodic points of period $\ell$ of $\mathcal{K}(\varphi) \in$ $\operatorname{Bir}\left(\mathbb{C}^{3}\right)_{\mathrm{c}(\omega)}$.

A similar question as Question 3 is the following:

QUestion 4. Let $\varphi$ be a polynomial automorphism of $\mathbb{C}^{2}$ of Hénon type; what is the topology of the distribution of periodic points of $\mathcal{K}(\varphi)$ ? Is it a discrete set ? Is its closure a Cantor set?

REMARK 4.2.5. Let us consider an element $\left(\phi_{0}\left(z_{0}, z_{1}\right), \phi_{1}\left(z_{0}, z_{1}\right), z_{2}+b\left(z_{0}, z_{1}\right)\right)$ of $\operatorname{Bir}\left(\mathbb{C}^{3}\right)_{\omega}$. Then $\phi_{t}=\left(\phi_{0}\left(z_{0}, z_{1}\right), \phi_{1}\left(z_{0}, z_{1}\right), z_{2}+b\left(z_{0}, z_{1}\right)+t\right)$ belongs to $\operatorname{Bir}\left(\mathbb{C}^{3}\right)_{\omega}$. If $p=\left(p_{0}, p_{1}, p_{2}\right)$ is a fixed point of $\phi_{t}$, then $\left(p_{0}, p_{1}\right)$ is a fixed point of $\varphi=\left(\phi_{0}, \phi_{1}\right)$ and $b\left(p_{0}, p_{1}\right)+t=0$. In particular if $\varphi$ only has isolated fixed points (that is $\varphi$ has no curve of fixed points, which is the case in general), then $\phi_{t}$ has no fixed points for $t$ generic.

Similarly, if $\varphi$ has a countable number of periodic points, then for $t$ generic $\phi_{t}$ has no periodic points.

We will look at degree and degree growths of some contact birational maps.

In the 2-dimensional case, that is if $\varphi$ belongs to $\operatorname{Aut}\left(\mathbb{C}^{2}\right)$, or $\operatorname{Bir}\left(\mathbb{P}^{2}\right)$, then $\operatorname{deg} \varphi=$ $\operatorname{deg} \varphi^{-1}$. This equality is not true in higher dimension; for instance if

$$
\phi=\left(z_{0}^{2}+z_{2}^{2}+z_{1}, z_{2}^{2}+z_{0}, z_{2}\right)
$$

then $\phi^{-1}=\left(z_{1}-z_{2}^{2}, z_{0}-\left(z_{1}-z_{2}^{2}\right)^{2}-z_{2}^{2}, z_{2}\right)$. What happens in our context ? The equality $\operatorname{deg} \varphi=\operatorname{deg} \varphi^{-1}$ still does not hold; indeed if $\left(\phi_{0}, \phi_{1}, z_{2}+b\left(z_{0}, z_{1}\right)\right)$ belongs to $\operatorname{Aut}\left(\mathbb{C}^{3}\right)_{\omega}$, then $-\mathrm{d} b=\phi_{0} \mathrm{~d} \phi_{1}-z_{0} \mathrm{~d} z_{1}$ and $\operatorname{deg} b=\operatorname{deg} \phi_{0}+\operatorname{deg} \phi_{1}$. For instance if $\varphi=\left(z_{0}+\left(z_{1}^{3}-z_{0}\right)^{2}, z_{1}^{3}-z_{0}\right)$, then

$$
\varphi^{-1}=\left(\left(z_{0}-z_{1}^{2}\right)^{3}-z_{1}, z_{0}-z_{1}^{2}\right) .
$$

Hence the degree of the $\varsigma$-lifts of $\varphi$ (resp. $\varphi^{-1}$ ) is 9 (resp. 8).

Let $\phi$ and $\psi$ be two birational self-maps of $\mathbb{P}^{3}$. We will say that the degree growths of $\phi$ and $\psi$ are of the same order if one of the following holds

- $\left(\operatorname{deg} \phi^{n}\right)_{n}$ and $\left(\operatorname{deg} \psi^{n}\right)_{n}$ are bounded,

- there exist an integer $k$ such that $\lim _{n \rightarrow+\infty} \operatorname{deg} \phi^{n} / n^{k}$ and $\lim _{n \rightarrow+\infty} \operatorname{deg} \psi^{n} / n^{k}$ are finite and nonzero,

- $\left(\operatorname{deg} \phi^{n}\right)_{n}$ and $\left(\operatorname{deg} \psi^{n}\right)_{n}$ grow exponentially. 
Let $\varphi$ be a polynomial automorphism of $\mathbb{C}^{2}$; let us recall that $\varphi$ has either a bounded growth or an exponential one $([\mathbf{1 9}])$. Denote by $\phi$ a $\varsigma$-lift of $\varphi$ to $\operatorname{Aut}\left(\mathbb{C}^{3}\right)_{\mathrm{c}(\omega)}$

$$
\phi=\left(\varphi, \operatorname{det} \operatorname{jac} \varphi z_{2}+b\left(z_{0}, z_{1}\right)\right) .
$$

Note that $b$ belongs to $\mathbb{C}\left[z_{0}, z_{1}\right]$ and so $\operatorname{deg} b\left(\varphi^{j}\left(z_{0}, z_{1}\right)\right) \leq \operatorname{deg} b \operatorname{deg} \varphi^{j}$ for any $j$. Hence

$$
\operatorname{deg} \varphi^{n} \leq \operatorname{deg} \phi^{n} \leq \max \left(\operatorname{deg} \varphi^{n}, \operatorname{deg} b \operatorname{deg} \varphi^{n-1}\right)
$$

and

- if $\left(\operatorname{deg} \varphi^{n}\right)_{n}$ is bounded, then $\left(\operatorname{deg} \phi^{n}\right)_{n}$ is bounded,

- if $\left(\operatorname{deg} \varphi^{n}\right)_{n}$ grows exponentially, then $\left(\operatorname{deg} \phi^{n}\right)_{n}$ grows exponentially.

Remark that if $\psi$ is a polynomial automorphism of $\mathbb{C}^{3}$ linear growth is also possible $([\mathbf{7}])$ and this eventuality does not appear when we look at elements of $\operatorname{Aut}\left(\mathbb{C}^{3}\right)_{c(\omega)}$.

In the case of the $\varsigma$-lift of an exact element of $\operatorname{Bir}\left(\mathbb{C}^{2}\right)_{\eta}$ we cannot give formula because we are not dealing with polynomials. But the degree growth of a $\varsigma$-lift $\phi$ of an exact element $\varphi$ of $\operatorname{Bir}\left(\mathbb{C}^{2}\right)_{\eta}$ and the degree growth of $\varphi$ are the same. Indeed set $\varphi^{n}=\left(\varphi_{0, n}, \varphi_{1, n}\right)$ for any $n \geq 1$. On the one hand

$$
\phi^{n}=\left(\varphi_{0, n}, \varphi_{1, n}, z_{2}+b\left(z_{0}, z_{1}\right)+b\left(\varphi_{0,1}, \varphi_{1,1}\right)+b\left(\varphi_{0,2}, \varphi_{1,2}\right)+\cdots+b\left(\varphi_{0, n-1}, \varphi_{1, n-1}\right)\right)
$$

with $\mathrm{d} b=z_{0} \mathrm{~d} z_{1}-\varphi_{0} \mathrm{~d} \varphi_{1}$, but on the other hand $\phi^{n}=\left(\varphi_{0, n}, \varphi_{1, n}, z_{2}+\widetilde{b}\left(z_{0}, z_{1}\right)\right)$ with $\mathrm{d} \widetilde{b}=z_{0} \mathrm{~d} z_{1}-\varphi_{0, n} \mathrm{~d} \varphi_{1, n}$. Using this last writing one gets the statement.

Let $\phi$ be a birational self-map of $\mathbb{P}^{2}$. For any $n \geq 1$ set $\phi^{n}=\left(\phi_{1, n}, \phi_{2, n}\right)=$ $\left(P_{1, n} / Q_{1, n}, P_{2, n} / Q_{2, n}\right)$ with $P_{i, n}, Q_{i, n} \in \mathbb{C}\left[z_{0}, z_{1}\right]$ without common factor; denote by $p_{i, q}$ (resp. $\left.q_{i, n}\right)$ the degree of $P_{i, n}$ (resp. $Q_{i, n}$ ). Of course $\operatorname{deg} \phi^{n}=\max \left(p_{1, n}+q_{2, n}, p_{2, n}+\right.$ $\left.q_{1, n}, q_{1, n}+q_{2, n}\right)$ and since

$$
\begin{aligned}
\mathcal{K}(\phi)^{n} & =\mathcal{K}\left(\phi^{n}\right) \\
& =\left(\frac{Q_{2, n}^{2}}{Q_{1, n}^{2}} \frac{P_{2, n} \frac{\partial Q_{2, n}}{\partial z_{1}}-Q_{2, n} \frac{\partial P_{2, n}}{\partial z_{1}}+\left(Q_{2, n} \frac{\partial P_{2, n}}{\partial z_{2}}-P_{2, n} \frac{\partial Q_{2, n}}{\partial z_{2}}\right) z_{0}}{Q_{1, n} \frac{\partial P_{1, n}}{\partial z_{1}}-P_{1, n} \frac{\partial Q_{1, n}}{\partial z_{1}}-\left(Q_{1, n} \frac{\partial P_{1, n}}{\partial z_{2}}-P_{1, n} \frac{\partial Q_{1, n}}{\partial z_{2}}\right) z_{0}}, \frac{P_{1, n}}{Q_{1, n}}, \frac{P_{2, n}}{Q_{2, n}}\right)
\end{aligned}
$$

one gets $\operatorname{deg} \phi^{n} \leq \operatorname{deg} \mathcal{K}(\phi)^{n} \leq \max \left(4 q_{2, n}+p_{2, n}+1,2 p_{1, n}+2 q_{1, n}+q_{2, n}+1, p_{2, n}+3 q_{1, n}+\right.$ $\left.p_{1, n}+1\right)$.

Proposition 4.2.6. - Assume that $\mathrm{G}=\operatorname{Aut}\left(\mathbb{C}^{2}\right)$ or $\mathrm{G}=\operatorname{Bir}\left(\mathbb{C}^{2}\right)_{\eta}$. Let $\varphi$ be an element of $\mathrm{G}$, and let $\phi$ be a $\varsigma$-lift of $\varphi$. The degree growths of $\varphi$ and $\phi$ are of the same order.

- Let $\varphi$ be a birational self-map of the complex projective plane, and let us consider $\mathcal{K}(\varphi)$ the image of $\varphi$ by $\mathcal{K}$. The degree growths of $\varphi$ and $\mathcal{K}(\varphi)$ are of the same order.

Let us end this section by some considerations about centralisers of contact birational maps. 
If $\mathrm{G}$ is a group and $f$ an element of $\mathrm{G}$, we denote by $\operatorname{Cent}(f, \mathrm{G})$ the centraliser of $f$ in $G$, that is

$$
\operatorname{Cent}(f, \mathrm{G})=\{g \in \mathrm{G} \mid f g=g f\} .
$$

Let $\varphi$ be a polynomial automorphism of $\mathbb{C}^{2}$, then $([\mathbf{1 9}][\mathbf{2 6}])$

- either $\varphi$ is conjugate to an element of $J_{2}$ and $\operatorname{Cent}\left(\varphi, \operatorname{Aut}\left(\mathbb{C}^{2}\right)\right)$ is uncountable;

- or $\varphi$ is of Hénon type and the centraliser of $\varphi$ is isomorphic to $\mathbb{Z} \rtimes \mathbb{Z} / p \mathbb{Z}$ for some $p$.

Let $\mathcal{H}$ be the set of polynomial automorphisms of $\mathbb{C}^{2}$ of Hénon type.

Proposition 4.2.7. Let $\varphi$ be a polynomial automorphism of $\mathbb{C}^{2}$ and let $\phi$ be one of its $\varsigma$-lift.

- If $\operatorname{det} \operatorname{jac} \varphi=1$, then $\operatorname{Cent}\left(\phi, \operatorname{Aut}\left(\mathbb{C}^{3}\right)_{\omega}\right)$ is uncountable and isomorphic to $\operatorname{Cent}(\phi) \rtimes \mathbb{C}$.

- If $\operatorname{det} \mathrm{jac} \varphi \neq 1$ and $\varphi$ belongs to $\mathcal{H}$, then $\operatorname{Cent}\left(\phi, \operatorname{Aut}\left(\mathbb{C}^{3}\right)_{\mathrm{c}(\omega)}\right)$ is countable and isomorphic to $\operatorname{Cent}(\varphi)$.

Proof. One can look at the restriction of $\varsigma$ to $\operatorname{Cent}\left(\phi, \operatorname{Aut}\left(\mathbb{C}^{3}\right)_{\mathrm{c}(\omega)}\right)$ :

$$
\varsigma_{\mid \operatorname{Cent}\left(\phi, \operatorname{Aut}\left(\mathbb{C}^{3}\right)_{\mathrm{c}(\omega)}\right)}: \operatorname{Cent}\left(\phi, \operatorname{Aut}\left(\mathbb{C}^{3}\right)_{\mathrm{c}(\omega)}\right) \rightarrow \operatorname{Cent}\left(\varphi, \operatorname{Aut}\left(\mathbb{C}^{2}\right)\right)
$$

Of course

$$
\operatorname{ker} \varsigma_{\mid \operatorname{Cent}\left(\phi, \operatorname{Aut}\left(\mathbb{C}^{3}\right)_{\mathrm{c}(\omega)}\right)} \subset\left\{\left(z_{0}, z_{1}, z_{2}+\beta\right) \mid \beta \in \mathbb{C}\right\} .
$$

If $\operatorname{det} \operatorname{jac} \varphi=1$, i.e. $\varphi$ belongs to $\operatorname{Aut}\left(\mathbb{C}^{2}\right)_{\eta}$, then

$$
\operatorname{ker} \varsigma_{\mid \operatorname{Cent}\left(\phi, \operatorname{Aut}\left(\mathbb{C}^{3}\right)_{\mathrm{c}(\omega)}\right)}=\left\{\left(z_{0}, z_{1}, z_{2}+\beta\right) \mid \beta \in \mathbb{C}\right\}
$$

and the centraliser of a $\varsigma$-lift of $\varphi$ is always uncountable even if $\operatorname{Cent}\left(\varphi, \operatorname{Aut}\left(\mathbb{C}^{2}\right)\right)$ is countable.

If $\operatorname{det} \operatorname{jac} \varphi \neq 1$, i.e. $\varphi$ belongs to $\operatorname{Aut}\left(\mathbb{C}^{2}\right) \backslash \operatorname{Aut}\left(\mathbb{C}^{2}\right)_{\eta}$, then $\operatorname{ker} \varsigma_{\mid \operatorname{Cent}\left(\phi, \operatorname{Aut}\left(\mathbb{C}^{3}\right)_{\mathrm{c}(\omega)}\right)}=$ $\{$ id $\}$ and

$$
\operatorname{Cent}\left(\phi, \operatorname{Aut}\left(\mathbb{C}^{3}\right)_{\mathrm{c}(\omega)}\right) \hookrightarrow \operatorname{Cent}\left(\varphi, \operatorname{Aut}\left(\mathbb{C}^{2}\right)\right)
$$

In particular if $\varphi$ belongs to $\left(\operatorname{Aut}\left(\mathbb{C}^{2}\right) \backslash \operatorname{Aut}\left(\mathbb{C}^{2}\right)_{\eta}\right) \cap \mathcal{H}$, then $\operatorname{Cent}\left(\phi, \operatorname{Aut}\left(\mathbb{C}^{3}\right)_{\mathrm{c}(\omega)}\right)$ is countable.

REMARK 4.2.8. Contrary to the 2-dimensional case there exist some $\phi$ in $\operatorname{Aut}\left(\mathbb{C}^{3}\right)_{\omega}$ such that

- $\operatorname{Cent}\left(\phi, \operatorname{Aut}\left(\mathbb{C}^{3}\right)_{\omega}\right)$ is uncountable,

- and $\left(\operatorname{deg} \phi^{n}\right)_{n \in \mathbb{N}}$ grows exponentially. 
A similar reasoning leads to:

Proposition 4.2.9. Let $\varphi \in \operatorname{Bir}\left(\mathbb{C}^{2}\right)_{\eta}$ be an exact map, and let $\phi$ be one of its $\varsigma$-lifts. Then $\operatorname{Cent}\left(\phi, \operatorname{Bir}\left(\mathbb{C}^{3}\right)_{\omega}\right)$ is uncountable.

Let $\mathrm{G}=\operatorname{Aut}\left(\mathbb{C}^{2}\right)$ or $\mathrm{G}=\operatorname{Bir}\left(\mathbb{C}^{2}\right)_{\eta}$. Let $\varphi$ be an element of $\mathrm{G}$, and let $\phi$ be one of its $\varsigma$-lift. In the following examples we look at the links between the $\varsigma$-lift of $\operatorname{Cent}(\varphi, G)$ and $\operatorname{Cent}\left(\phi, \mathrm{G}^{\prime}\right)$ where $\mathrm{G}^{\prime}=\operatorname{Aut}\left(\mathbb{C}^{3}\right)_{\mathrm{c}(\omega)}$ or $\operatorname{Bir}\left(\mathbb{C}^{3}\right)_{\mathrm{c}(\omega)}$.

EXAMPLE 4.2.10. In this example we give a polynomial automorphism $\varphi$ and maps in $\operatorname{Cent}\left(\varphi, \operatorname{Aut}\left(\mathbb{C}^{2}\right)\right)$ whose only one $\varsigma$-lift belongs to $\operatorname{Aut}\left(\phi, \operatorname{Aut}\left(\mathbb{C}^{3}\right)_{\mathrm{c}(\omega)}\right)$ where $\phi$ denotes a $\varsigma$-lift of $\varphi$.

Let us now consider the Hénon automorphism $\varphi$ given by

$$
\varphi=\left(\delta z_{1}, \beta z_{1}^{k}-\gamma z_{0}\right)
$$

where $\delta, \beta, \gamma$ are complex numbers such that $\delta \beta \neq 0, \delta \beta \neq 1$ and $k \geq 4$. The map

$$
\phi=\left(\delta z_{1}, \beta z_{1}^{k}-\gamma z_{0}, \delta \gamma z_{2}+\delta \gamma z_{0} z_{1}-\frac{\delta \beta}{k+1} z_{1}^{k+1}\right)
$$

is a $\varsigma$-lift of $\varphi$. One can check that $\left(\zeta z_{0}, \zeta z_{1}\right)$, where $\zeta \in \mathbb{C}^{*}$ such that $\zeta^{k}=\zeta$, commutes with $\varphi$. Among the $\varsigma$-lifts $\left(\zeta z_{0}, \zeta z_{1}, \zeta^{2} z_{2}+\beta\right), \beta \in \mathbb{C}$, only one commutes with $\phi$.

EXAMPle 4.2.11. We consider a polynomial automorphism $\varphi$, a subgroup $G$ of $\operatorname{Cent}\left(\varphi, \operatorname{Aut}\left(\mathbb{C}^{2}\right)\right)$ and $\mathrm{G}_{\varsigma}$ its $\varsigma$-lift. In the first example the inclusion $\mathrm{G}_{\varsigma} \subset$ $\operatorname{Cent}\left(\phi, \operatorname{Aut}\left(\mathbb{C}^{3}\right)_{\mathrm{c}(\omega)}\right)$ holds whereas in the second example it doesn't.

Let us consider the polynomial automorphism $\varphi=\left(\beta^{d} z_{0}+\beta^{d} z_{1}^{d} Q\left(z_{1}^{r}\right), \beta z_{1}\right)$ with $\beta \in \mathbb{C}^{*}, Q \in \mathbb{C}\left[z_{1}\right]$ and $d, r \in \mathbb{N}$. One can check that

$$
\mathrm{G}=\left\{\left(z_{0}+\gamma z_{1}^{d}, z_{1}\right) \mid \gamma \in \mathbb{C}\right\} \subset \operatorname{Cent}\left(\varphi, \operatorname{Aut}\left(\mathbb{C}^{2}\right)\right)
$$

The map $\phi=\left(\beta^{d} z_{0}+\beta^{d} z_{1}^{d} Q\left(z_{1}^{r}\right), \beta z_{1}, \beta^{d+1} z_{2}-\beta P\left(z_{1}\right)\right)$ with $P^{\prime}\left(z_{1}\right)=z_{1}^{q} Q\left(z_{1}^{r}\right)$ is a $\varsigma$-lift of $\varphi$. Let $\mathrm{G}_{\varsigma}$ be the $\varsigma$-lift of $\mathrm{G}$; the group

$$
\mathrm{G}_{\varsigma}=\left\{\left(z_{0}+\gamma z_{1}^{d}, z_{1}, z_{2}-\frac{\gamma z_{1}^{d+1}}{d+1}\right) \mid \gamma \in \mathbb{C}\right\}
$$

is here contained in $\operatorname{Cent}\left(\phi, \operatorname{Aut}\left(\mathbb{C}^{3}\right)_{\mathrm{c}(\omega)}\right)$.

Let $\varphi$ be the polynomial automorphism given by $\varphi=\left(z_{0}+z_{1}^{2}, \lambda z_{1}\right)$ with $\lambda \in \mathbb{C}^{*}$ and $\lambda^{2} \neq 1$. A $\varsigma$-lift of $\varphi$ to $\operatorname{Aut}\left(\mathbb{C}^{3}\right)_{\mathrm{c}(\omega)}$ is

$$
\phi=\left(z_{0}+z_{1}^{2}, \lambda z_{1}, \lambda z_{2}-\frac{z_{1}^{3}}{3}+\mu\right)
$$

for some $\mu \in \mathbb{C}$. Note that

$$
\mathrm{G}=\left\{\left(\delta z_{0}+\frac{\gamma^{2}-\delta}{\lambda^{2}-1} z_{1}+\varepsilon, \gamma z_{1}\right) \mid \delta, \gamma \in \mathbb{C}^{*}, \varepsilon \in \mathbb{C}\right\}
$$


is contained in $\operatorname{Cent}\left(\varphi, \operatorname{Aut}\left(\mathbb{C}^{2}\right)\right)$. Let us denote by $\mathrm{G}_{\varsigma}$ the $\varsigma$-lift of $\mathrm{G}$; a direct computation shows that

$\mathrm{G}_{\varsigma}=\left\{\left(\delta z_{0}+\frac{\gamma^{2}-\delta}{\lambda^{2}-1} z_{1}+\varepsilon, \gamma z_{1}, \delta \gamma z_{2}-\frac{\gamma\left(\gamma^{2}-\delta\right)}{3\left(\lambda^{2}-1\right)} z_{1}^{3}-\gamma \varepsilon z_{1}+\beta\right) \mid \delta, \gamma \in \mathbb{C}^{*}, \beta, \varepsilon \in \mathbb{C}\right\}$.

The inclusion $\mathrm{G}_{\varsigma} \cap \operatorname{Cent}\left(\phi, \operatorname{Aut}\left(\mathbb{C}^{3}\right)_{\mathrm{c}(\omega)}\right) \subsetneq \mathrm{G}_{\varsigma}$ is strict; indeed

$\mathrm{G}_{\varsigma} \cap \operatorname{Cent}\left(\phi, \operatorname{Aut}\left(\mathbb{C}^{3}\right)_{\mathrm{c}(\omega)}\right)=\left\{\left(\gamma^{2} z_{0}+\varepsilon, \gamma z_{1}, \gamma^{3} z_{2}-\gamma \varepsilon z_{1}+\frac{\gamma^{3}-1}{\lambda-1} \delta\right) \mid \gamma \in \mathbb{C}^{*}, \varepsilon \in \mathbb{C}\right\}$.

\subsection{Non-simplicity, Tits alternative.}

Let us recall that a simple group is a non-trivial group $\mathrm{G}$ whose only normal subgroups are $\{\mathrm{id}\}$ and $\mathrm{G}$.

Danilov proved that Aut $\left(\mathbb{C}^{2}\right)_{\eta}$ is not simple $([\mathbf{1 5}])$. More recently Cantat and Lamy showed that $\operatorname{Bir}\left(\mathbb{P}^{2}\right)$ is not simple $([\mathbf{1 1}])$. As a consequence one has:

Proposition 4.3.1. The groups

$$
\operatorname{Aut}\left(\mathbb{C}^{3}\right)_{\omega}, \operatorname{Bir}\left(\mathbb{C}^{3}\right)_{\omega}, \operatorname{Aut}\left(\mathbb{C}^{3}\right)_{\mathrm{c}(\omega)},\left[\operatorname{Aut}\left(\mathbb{C}^{3}\right)_{\mathrm{c}(\omega)}, \operatorname{Aut}\left(\mathbb{C}^{3}\right)_{\mathrm{c}(\omega)}\right],\left[\operatorname{Aut}\left(\mathbb{C}^{3}\right)_{\omega}, \operatorname{Aut}\left(\mathbb{C}^{3}\right)_{\omega}\right]
$$

are not simple.

Proof. Since $\left[\operatorname{Aut}\left(\mathbb{C}^{3}\right)_{\mathrm{c}(\omega)}, \operatorname{Aut}\left(\mathbb{C}^{3}\right)_{\mathrm{c}(\omega)}\right] \simeq \operatorname{Aut}\left(\mathbb{C}^{2}\right)_{\eta}$ and $\left[\operatorname{Aut}\left(\mathbb{C}^{3}\right)_{\omega}, \operatorname{Aut}\left(\mathbb{C}^{3}\right)_{\omega}\right]$ $\simeq \operatorname{Aut}\left(\mathbb{C}^{2}\right)_{\eta}$ the first assertion follows from $[\mathbf{1 5}]$.

The exact sequence (2.1) implies in particular that there exists a morphism with a non-trivial kernel from Aut $\left(\mathbb{C}^{3}\right)_{\omega}$ into Aut $\left(\mathbb{C}^{2}\right)_{\eta}$, hence $\operatorname{Aut}\left(\mathbb{C}^{3}\right)_{\omega}$ is not simple. A similar argument holds for $\operatorname{Bir}\left(\mathbb{C}^{3}\right)_{\omega}$ and $\operatorname{Aut}\left(\mathbb{C}^{3}\right)_{\mathrm{c}(\omega)}$.

The morphism

$$
\operatorname{Bir}\left(\mathbb{C}^{3}\right)_{\omega}^{\mathrm{reg}} \longrightarrow \operatorname{Bir}\left(\mathbb{P}^{2}\right)
$$

that consists to take the restriction of $\phi \in \operatorname{Bir}\left(\mathbb{C}^{3}\right)_{\omega}^{\text {reg }}$ to $\mathcal{H}_{\infty}$ has a non-trivial kernel; indeed

$$
\phi=\left(z_{0}-\left(\frac{P\left(z_{1}\right)}{Q\left(z_{1}\right)}\right)^{\prime}, z_{1}, z_{2}+\frac{P\left(z_{1}\right)}{Q\left(z_{1}\right)}\right)
$$

with $P, Q$ two polynomials of degree $p, q$ such that $p<q+1$, is regular and induces the identity on $\mathcal{H}_{\infty}$. In particular one gets the following statement:

Proposition 4.3.2. The group $\operatorname{Bir}\left(\mathbb{C}^{3}\right)_{\omega}^{\mathrm{reg}}$ is not simple.

Let us consider the maps $\psi=\left(\gamma z_{0}^{2} z_{1}, 1 / \gamma z_{0}, z_{2}+z_{0} z_{1}\right)$ and $\phi=\left(z_{0}+1 / z_{1}^{3}, z_{1}, z_{2}+\right.$ $\left.1 / 2 z_{1}^{2}\right)$. One can check that $\psi$ belongs to $\operatorname{Bir}\left(\mathbb{C}^{3}\right)_{\omega} \backslash \operatorname{Bir}\left(\mathbb{C}^{3}\right)_{\omega}^{\text {reg }}$ whereas $\phi$ is in $\operatorname{Bir}\left(\mathbb{C}^{3}\right)_{\omega}^{\text {reg }}$. A direct computation shows that $\psi^{-1} \phi \psi$ blows down $\mathcal{H}_{\infty}$ onto $\boldsymbol{e}_{3}$. Hence one can state:

Proposition 4.3.3. The subgroup $\operatorname{Bir}\left(\mathbb{C}^{3}\right)_{\omega}^{\mathrm{reg}}$ of $\operatorname{Bir}\left(\mathbb{C}^{3}\right)_{\omega}$ is not normal. 
We will end this section by establishing Tits Alternative for $\operatorname{Aut}\left(\mathbb{C}^{3}\right)_{\omega}, \operatorname{Aut}\left(\mathbb{C}^{3}\right)_{\mathrm{c}(\omega)}$ and $\operatorname{Bir}\left(\mathbb{C}^{3}\right)_{\omega}$.

The derived series of a group $\mathrm{G}$ is defined as follows

$$
D_{0}(\mathrm{G})=\mathrm{G}, \quad D_{1}(\mathrm{G})=[\mathrm{G}, \mathrm{G}], \quad \ldots, \quad D_{n+1}(\mathrm{G})=\left[\mathrm{G}, D_{n}(\mathrm{G})\right] .
$$

The group $\mathrm{G}$ is solvable if there exists an integer $k$ such that $D_{k}(\mathrm{G})=\{\mathrm{id}\}$. The least $\ell$ such that $D_{\ell}=\{\mathrm{id}\}$ is called the derived length of $\mathrm{G}$.

A group $\mathrm{G}$ satisfies the Tits alternative if any finitely generated subgroup of $\mathrm{G}$ contains either a non-abelian free group, or a solvable subgroup of finite index. This alternative has been established by Tits for linear groups $G L(n ; \mathbb{k})$ for any field $\mathbb{k}([\mathbf{2 8}])$. Lamy proves that the group of polynomial automorphisms of $\operatorname{Aut}\left(\mathbb{C}^{2}\right)$ satisfies the Tits alternative $([\mathbf{2 6}])$, so does Cantat for the group of birational maps of a complex, compact, kähler surface (see $[\mathbf{1 0}]$ ). Note that the automorphisms groups of complex, compact, kähler manifolds of any dimension also satisfy Tits alternative ([10][27]).

TheOrem 4.3.4. The groups $\operatorname{Aut}\left(\mathbb{C}^{3}\right)_{\omega}$, Aut $\left(\mathbb{C}^{3}\right)_{\mathrm{c}(\omega)}$ and $\operatorname{Bir}\left(\mathbb{C}^{3}\right)_{\omega}$ satisfy the Tits alternative.

Proof. Let $\mathrm{G}$ be a finitely generated subgroup of $\operatorname{Bir}\left(\mathbb{C}^{3}\right)_{\omega}$. Set

$$
\mathrm{G}_{0}=\varsigma(\mathrm{G}) \subset \operatorname{Bir}\left(\mathbb{C}^{2}\right)_{\eta}
$$

Since $\operatorname{Bir}\left(\mathbb{C}^{2}\right)_{\eta}$ is a subgroup of $\operatorname{Bir}\left(\mathbb{P}^{2}\right)$ that satisfies the Tits alternative, either $\mathrm{G}_{0}$ contains a non-abelian free group, or a solvable subgroup of finite index.

Assume first that $\mathrm{G}_{0}$ contains two elements $f$ and $h$ such that $\langle f, h\rangle \simeq \mathbb{Z} * \mathbb{Z}$. Let us denote by $F$, resp. $H$ a lift of $f$, resp. $h$ in $\operatorname{Bir}\left(\mathbb{P}^{3}\right)$. Suppose that there exists a non-trivial word $M$ such that $M(F, H)=\{\mathrm{id}\}$. As $\varsigma$ is a morphism, one gets that $M(f, h)=\{\mathrm{id}\}$ : contradiction.

Suppose now that up to finite index $\mathrm{G}_{0}$ is solvable, and let $\ell$ be its derived length; in particular $D_{\ell}\left(\mathrm{G}_{0}\right)=\{\mathrm{id}\}$ and $D_{\ell}(\mathrm{G})$ belongs to ker $\varsigma$. Since

$$
\operatorname{ker} \varsigma=\left\{\left(z_{0}, z_{1}, z_{2}+\beta\right) \mid \beta \in \mathbb{C}\right\}
$$

one gets $D_{\ell+1}(\mathrm{G})=\{\mathrm{id}\}$.

\subsection{Non-conjugate isomorphic groups.}

Let us denote by $v_{1}$ the trivial embedding from $\left(\operatorname{Aut}\left(\mathbb{C}^{2}\right)_{\eta} \mid 0\right)$ into $\operatorname{Aut}\left(\mathbb{C}^{3}\right)$

$$
v_{1}:\left(\operatorname{Aut}\left(\mathbb{C}^{2}\right)_{\eta} \mid 0\right) \hookrightarrow \operatorname{Aut}\left(\mathbb{C}^{3}\right), \quad\left(\phi_{0}, \phi_{1}\right) \mapsto\left(\phi_{0}, \phi_{1}, z_{2}\right)
$$

and by $v_{2}$ the trivial embedding from $\operatorname{Bir}\left(\mathbb{P}^{2}\right)$ into $\operatorname{Bir}\left(\mathbb{P}^{3}\right)$

$$
v_{2}: \operatorname{Bir}\left(\mathbb{P}^{2}\right) \hookrightarrow \operatorname{Bir}\left(\mathbb{P}^{3}\right), \quad\left(\phi_{1}, \phi_{2}\right) \mapsto\left(z_{0}, \phi_{1}, \phi_{2}\right) .
$$

Despite $\operatorname{im} v_{1}\left(\right.$ resp. $\left.\operatorname{im} v_{2}\right)$ is isomorphic to $\operatorname{im} \varsigma($ resp. $\operatorname{im} \mathcal{K})$ one has the following statement: 
Proposition 4.4.1. The image of $v_{1}$ (resp. $\left.v_{2}\right)$ is not Aut $\left(\mathbb{C}^{3}\right)$-conjugate (resp. $\operatorname{Bir}\left(\mathbb{P}^{3}\right)$-conjugate) to a subgroup of $\operatorname{Aut}\left(\mathbb{C}^{3}\right)_{\mathrm{c}(\omega)}\left(\right.$ resp. $\left.\operatorname{Bir}\left(\mathbb{C}^{3}\right)_{\mathrm{c}(\omega)}\right)$.

Proof. Let us assume that there exists $\psi$ in $\operatorname{Aut}\left(\mathbb{C}^{3}\right)\left(\right.$ resp. $\left.\operatorname{Bir}\left(\mathbb{P}^{3}\right)\right)$ such that for any $\phi=\left(\phi_{0}, \phi_{1}\right)\left(\right.$ resp. $\left.\phi=\left(\phi_{1}, \phi_{2}\right)\right)$ in $\operatorname{Aut}\left(\mathbb{C}^{2}\right)\left(\operatorname{resp} . \operatorname{Bir}\left(\mathbb{P}^{2}\right)\right)$ the map $\psi v_{1}(\phi) \psi^{-1}$ (resp. $\psi v_{2}(\phi) \psi^{-1}$ ) is a contact polynomial automorphism (resp. contact birational map); as a result $v_{1}(\phi)$ (resp. $\left.v_{2}(\phi)\right)$ preserves a polynomial form $\Theta=A \mathrm{~d} z_{0}+B \mathrm{~d} z_{1}+C \mathrm{~d} z_{2}$. Looking at the restriction to any hyperplane $z_{2}=\lambda$ (resp. $z_{0}=\lambda$ ) for $\lambda$ generic one gets that all the $\phi$ preserve the foliation given by $\Theta_{\mid z_{2}=\lambda}\left(\right.$ resp. $\left.\Theta_{\mid z_{0}=\lambda}\right)$ : contradiction.

\section{Appendix: Automorphisms group of $\operatorname{Aut}\left(\mathbb{C}^{2}\right)_{\eta}$.}

As we recalled Aut $\left(\mathbb{C}^{2}\right)$ is generated by $\mathrm{J}_{2}$ and $\mathrm{Aff}_{2}$. More precisely $\operatorname{Aut}\left(\mathbb{C}^{2}\right)$ has a structure of amalgamated product $([\mathbf{2 5}])$

$$
\operatorname{Aut}\left(\mathbb{C}^{2}\right)=\mathrm{J}_{2} * \mathrm{~J}_{2} \cap \mathrm{Aff}_{2} \mathrm{Aff}_{2}
$$

this is also the case for $\operatorname{Aut}\left(\mathbb{C}^{2}\right)_{\eta}([\mathbf{2 0}$, Proposition 9])

$$
\operatorname{Aut}\left(\mathbb{C}^{2}\right)_{\eta}=\left(\mathrm{J}_{2}\right)_{\eta} *\left(\mathrm{~J}_{2}\right)_{\eta} \cap\left(\operatorname{Aff}_{2}\right)_{\eta}\left(\mathrm{Aff}_{2}\right)_{\eta} .
$$

Following [16] we prove that:

THEOREM 5.0.2. The group Aut $\left(\operatorname{Aut}\left(\mathbb{C}^{2}\right)_{\eta}\right)$ is generated by the automorphisms of the field $\mathbb{C}$ and the group of Aut $\left(\mathbb{C}^{2}\right)$-inner automorphisms.

IdeA of The Proof. Let us set $\mathcal{G}=\operatorname{Aut}\left(\mathbb{C}^{2}\right)_{\eta}$. One can follow $[\mathbf{1 6}]$ and prove that if $\varphi$ is an automorphism of $\mathcal{G}$, then

- $\varphi\left(\left(\mathrm{J}_{2}\right)_{\eta}\right)=\left(\mathrm{J}_{2}\right)_{\eta}$ up to conjugacy by an element of $\operatorname{Aut}\left(\mathbb{C}^{2}\right)([\mathbf{1 6}$, Proposition 4.4$])$;

- for any integer $k$ if $\mathcal{R}=\cup_{n \leq k}\left\langle\left(\beta z_{0}, z_{1} / \beta\right)\right| \beta n$-th root of unity $\rangle$, then there exists $\psi$ in $\left(\mathrm{J}_{2}\right)_{\eta}$ such that $\varphi(\mathcal{R})=\psi \mathcal{R} \psi^{-1}$. So one can suppose that $\varphi\left(\left(\mathrm{J}_{2}\right)_{\eta}\right)=\left(\mathrm{J}_{2}\right)_{\eta}$ and $\varphi(\mathcal{R})=\mathcal{R}$ (see [16, Proposition 4.4]);

- set $\mathrm{D}_{\eta}=\left\{\left(\beta z_{0}, z_{1} / \beta\right) \mid \beta \in \mathbb{C}^{*}\right\}$ one can show that conjugating $\phi$ by an element of $\left(\mathrm{J}_{2}\right)_{\eta}$ one has $\varphi\left(\left(\mathrm{J}_{2}\right)_{\eta}\right)=\left(\mathrm{J}_{2}\right)_{\eta}$ and $\varphi\left(\mathrm{D}_{\eta}\right)=\mathrm{D}_{\eta}$.

- set

$$
\mathrm{T}_{1}=\left\{\left(z_{0}+\beta, z_{1}\right) \mid \beta \in \mathbb{C}\right\}, \quad \mathrm{T}_{2}=\left\{\left(z_{0}, z_{1}+\beta\right) \mid \beta \in \mathbb{C}\right\}
$$

and

$$
\mathrm{T}=\left\{\left(z_{0}+\gamma, z_{1}+\beta\right) \mid \gamma, \beta \in \mathbb{C}\right\} .
$$

Since $\mathrm{T}_{1} \subset\left[\left[\left(\mathrm{J}_{2}\right)_{\eta},\left(\mathrm{J}_{2}\right)_{\eta}\right],\left[\left(\mathrm{J}_{2}\right)_{\eta},\left(\mathrm{J}_{2}\right)_{\eta}\right]\right]$, then $\mathrm{T}_{1} \subset\left\{\left(z_{0}+P\left(z_{1}\right), z_{1}\right) \mid P \in \mathbb{C}\left[z_{1}\right]\right\}$. As

$$
\forall n \in \mathbb{N}, \forall \beta \in \mathbb{C} \quad\left(\frac{z_{0}}{n}, n z_{1}\right)\left(z_{0}+\beta, z_{1}\right)^{n}\left(n z_{0}, \frac{z_{1}}{n}\right)=\left(z_{0}+\beta, z_{1}\right)
$$


and $\varphi\left(\mathrm{D}_{\eta}\right)=\mathrm{D}_{\eta}$, one gets

$$
\forall n \in \mathbb{N}, \forall \beta \in \mathbb{C} \quad \varphi\left(\frac{z_{0}}{n}, n z_{1}\right) \varphi\left(z_{0}+\beta, z_{1}\right)^{n} \varphi\left(n z_{0}, \frac{z_{1}}{n}\right)=\varphi\left(z_{0}+\beta, z_{1}\right)
$$

that is

$$
\forall n \in \mathbb{N} \quad\left(\frac{z_{0}}{\delta}, \delta z_{1}\right)\left(z_{0}+n P\left(z_{1}\right), z_{1}\right)^{n}\left(\delta z_{0}, \frac{z_{1}}{\delta}\right)=\left(z_{0}+P(z), z_{1}\right)
$$

so $P\left(z_{1}\right)=n / \delta P\left(z_{1} / \delta\right)$. The polynomial $P$ is non-zero hence $n=\delta$ and $P$ is a constant. Therefore $\varphi\left(\mathrm{T}_{1}\right) \subset \mathrm{T}_{1}$.

The groups $\mathrm{T}_{1}$ and $\mathrm{T}_{2}$ commute, that's why

$$
\varphi\left(\mathrm{T}_{2}\right) \subset\left\{\left(z_{0}+P\left(z_{1}\right), z_{1}+\beta\right) \mid P \in \mathbb{C}\left[z_{1}\right], \beta \in \mathbb{C}\right\} .
$$

The relation

$$
\left(\frac{z_{0}}{n}, n z_{1}\right)\left(z_{0}, z_{1}+\beta\right)\left(n z_{0}, \frac{z_{1}}{n}\right)=\left(z_{0}, z_{1}+\beta\right)^{n}
$$

true for any integer $n$ and for any $\beta$ in $\mathbb{C}$ implies that $\varphi\left(\mathrm{T}_{2}\right) \subset \mathrm{T}_{2}$. The group $\mathrm{T}$ being a maximal abelian subgroup of $\mathcal{G}$, one has $\varphi(\mathrm{T})=\mathrm{T}$ and $\varphi\left(\mathrm{T}_{i}\right)=\mathrm{T}_{i}$.

- There exist $\xi_{1}, \xi_{2}$ two additive morphisms and $\zeta$ a multiplicative one such that

$$
\varphi\left(z_{0}+\gamma, z_{1}+\beta\right)=\left(z_{0}+\xi_{1}(\gamma), z_{1}+\xi_{2}(\beta)\right) \quad \& \quad \varphi\left(\gamma z_{0}, \frac{z_{1}}{\gamma}\right)=\left(\zeta(\gamma) z_{0}, \frac{z_{1}}{\zeta(\gamma)}\right)
$$

The statement follows from [16, Proposition 1.4].

\section{References}

[1] M. Alberich-Carramiñana, Geometry of the plane Cremona maps, Lecture Notes in Math., 1769, Springer-Verlag, Berlin, 2002.

[ 2 ] A. Banyaga, Sur le groupe des difféomorphismes symplectiques, C. R. Acad. Sci. Paris Sér. A, 278 (1974), 1343-1344.

[ 3 ] A. Banyaga, Sur le groupe des difféomorphismes qui préservent une forme de contact régulière, C. R. Acad. Sci. Paris Sér. A-B, 281 (1975), A707-A709.

[4] A. Banyaga, The group of diffeomorphisms preserving a regular contact form, In: Topology and algebra (Proc. Colloq., Eidgenöss. Tech. Hochsch., Zurich, 1977), Monograph. Enseign. Math., 26, Univ. Genève, Geneva, 1978, 47-53.

[ 5 ] L. Bayle and A. Beauville, Birational involutions of $\mathbf{P}^{2}$, Asian J. Math., 4 (2000), Kodaira's issue, $11-17$.

[6 ] E. Bertini, Ricerche sulle trasformazioni univoche involutorie nel piano, Annali di Mat., 8 (1877), 244-286.

[7] A. M. Bonifant and J. E. Fornæss, Growth of degree for iterates of rational maps in several variables, Indiana Univ. Math. J., 49 (2000), 751-778.

[ 8 ] M. Brunella, Minimal models of foliated algebraic surfaces, Bull. Soc. Math. France, 127 (1999), 289-305.

[ 9 ] S. Cantat, Dynamique des automorphismes des surfaces complexes compactes, PhD thesis, École Normale Supérieure de Lyon, 1999.

[10] S. Cantat, Sur les groupes de transformations birationnelles des surfaces, Ann. of Math. (2), 174 (2011), 299-340. 
[11] S. Cantat and S. Lamy, Normal subgroups in the Cremona group, Acta Math., 210 (2013), 31-94.

[12] G. Casale, Le groupoïde de Galois de $P_{1}$ et son irréductibilité, Comment. Math. Helv., 83 (2008), $471-519$.

[13] D. Cerveau and J. Déserti, Transformations birationnelles de petit degré, Cours Spécialisés, 19, Société Mathématique de France, Paris, 2013.

[14] D. Cerveau and J.-F. Mattei, Formes intégrables holomorphes singulières, Astérisque, 97, Société Mathématique de France, Paris, 1982.

[15] V. I. Danilov, Non-simplicity of the group of unimodular automorphisms of an affine plane, Mat. Zametki, 15 (1974), 289-293.

[16] J. Déserti, Sur le groupe des automorphismes polynomiaux du plan affine, J. Algebra, 297 (2006), $584-599$.

[17] J. Déserti, Sur les automorphismes du groupe de Cremona, Compos. Math., 142 (2006), 14591478.

[18] K. Frantzen and T. Peternell, On the bimeromorphic geometry of compact complex contact threefolds, In: Classification of algebraic varieties, EMS Ser. Congr. Rep., Eur. Math. Soc., Zürich, 2011, 277-288.

[19] S. Friedland and J. Milnor, Dynamical properties of plane polynomial automorphisms, Ergodic Theory Dynam. Systems, 9 (1989), 67-99.

[20] J.-P. Furter and S. Lamy, Normal subgroup generated by a plane polynomial automorphism, Transform. Groups, 15 (2010), 577-610.

[21] M. Gizatullin, Klein's conjecture for contact automorphisms of the three-dimensional affine space, Michigan Math. J., 56 (2008), 89-98.

[22] M. H. Gizatullin and V. I. Danilov, Automorphisms of affine surfaces, I. Izv. Akad. Nauk SSSR Ser. Mat., 39 (1975), 523-565, 703.

[23] E. L. Ince, Ordinary Differential Equations, Dover Publications, New York, 1944.

[24] J. P. Jouanolou, Équations de Pfaff algébriques, Lecture Notes in Math., 708, Springer, Berlin, 1979.

[25] H. W. E. Jung, Über ganze birationale Transformationen der Ebene, J. Reine Angew. Math., 184 (1942), 161-174.

[26] S. Lamy, L'alternative de Tits pour Aut[ $\left.\mathbb{C}^{2}\right]$, J. Algebra, 239 (2001), 413-437.

[27] K. Oguiso, Tits alternative in hyperkähler manifolds, Math. Res. Lett., 13 (2006), 307-316.

[28] J. Tits, Free subgroups in linear groups, J. Algebra, 20 (1972), 250-270.

Dominique Cerveau

Université de Rennes 1

CNRS, IRMAR-UMR 6625

F-Rennes, France

E-mail: dominique.cerveau@univ-rennes1.fr

\section{Julie DÉSERTI}

Université Paris Diderot

Sorbonne Paris Cité

Institut de Mathémtiques de

Jussieu-Paris Rive Gauche

UMR 7586, CNRS

Sorbonne Universités

UPMC Univ Pris 06

F-75013 Paris, France

E-mail: deserti@math.univ-paris-diderot.fr 